\title{
Cortical processing of pain : a bridge between experimental findings and clinical implications
}

Citation for published version (APA):

Vossen, H. G. M. (2010). Cortical processing of pain : a bridge between experimental findings and clinical implications. [Doctoral Thesis, Maastricht University]. Maastricht University. https://doi.org/10.26481/dis.20100331hv

Document status and date:

Published: 01/01/2010

DOI:

10.26481/dis.20100331hv

Document Version:

Publisher's PDF, also known as Version of record

\section{Please check the document version of this publication:}

- A submitted manuscript is the version of the article upon submission and before peer-review. There can be important differences between the submitted version and the official published version of record.

People interested in the research are advised to contact the author for the final version of the publication, or visit the DOI to the publisher's website.

- The final author version and the galley proof are versions of the publication after peer review.

- The final published version features the final layout of the paper including the volume, issue and page numbers.

Link to publication

\footnotetext{
General rights rights.

- You may freely distribute the URL identifying the publication in the public portal. please follow below link for the End User Agreement:

www.umlib.nl/taverne-license

Take down policy

If you believe that this document breaches copyright please contact us at:

repository@maastrichtuniversity.nl

providing details and we will investigate your claim.
}

Copyright and moral rights for the publications made accessible in the public portal are retained by the authors and/or other copyright owners and it is a condition of accessing publications that users recognise and abide by the legal requirements associated with these

- Users may download and print one copy of any publication from the public portal for the purpose of private study or research.

- You may not further distribute the material or use it for any profit-making activity or commercial gain

If the publication is distributed under the terms of Article $25 \mathrm{fa}$ of the Dutch Copyright Act, indicated by the "Taverne" license above, 


\section{CORTICAL PROCESSING OF PAIN:}

\section{A BRIDGE BETWEEN EXPERIMENTAL FINDINGS AND CLINICAL}

IMPLICATIONS 
Cover image designed by Vanessa Verbeeck and based on an image of E. Ansel "Pain of the Blues" This thesis was printed on a HP Digital Press

ISBN 978-90-902-5148-6

(C) Helen Vossen, Maastricht, the Netherlands, 2010

All rights reserved. No part of this book may be reproduced, stored in a retrieval system, or transmitted, in any form or by any means, electronic, mechanical, photocopying, recording, or otherwise, without the prior written permission of the holder of the copyright 


\title{
CORTICAL PROCESSING OF PAIN:
}

\section{a bridge between experimental findings and clinical implications}

\author{
PROEFSCHRIFT
}

ter verkrijging van de graad van doctor aan de Universiteit Maastricht, op gezag van de Rector Magnificus, Prof mr. G.P.M.F. Mols volgens het besluit van het College van Decanen, in het openbaar te verdedigen op woensdag 31 maart 2010 om 12.00 uur

door

Hélène Guillaume Marie Vossen

Geboren op 26 januari 1981 te Geleen 


\section{Promotoren:}

Prof. dr. J.van Os

Prof. dr. ir. H.J. Hermens (Universiteit Twente)

\section{Copromoter:}

Dr. R. Lousberg

\section{Beoordelingscommissie:}

Prof. dr. F.R.J. Verhey (voorzitter)

Prof. dr. M.I. Hasenbring (Ruhr-Universität Bochum)

Prof. dr. M.M.R Hutten-Vollenbroek (Universiteit Twente)

Dr. F.T.Y Smulders

Dr. J.J.M.H. Strik

The research presented in this thesis was conducted at the Department of Psychiatry and Neuropsychology of Maastricht University and the Roessingh Research and Development in Enschede. 
Voor mijn opa 
Paranimfen:

Carine Vossen

Christine Vossen 


\section{Table of Contents}

Evidence that trait-anxiety and trait-depression differentially moderate cortical processing of pain.

Chapter 3

More potential in statistical analyses of event-related potentials:

A multilevel approach

\section{Chapter 4}

A challenge to the notion of a clear-cut association between

Event-Related Potentials and subjective pain ratings:

An experimental study using multilevel analyses

Chapter 5

Cortical differences in habituation to experimental pain between chronic low back pain sufferers and pain free controls

Chapter 6

The influence of COMT 158ValMet, BDNF Val66Met and OPRM1 A118G polymorphisms on the cortical processing of experimental pain

Chapter 7

The predictive value of pain Event-Related Potentials for clinical experience of pain

General Discussion

Summary 
Dankwoord

151

List of Publications

153 


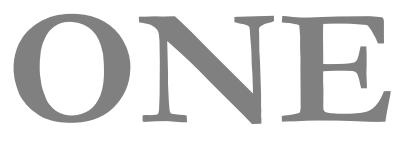

\section{General Introduction}




\section{Background}

The principle goal of science is to develop models that describe nature. The information obtained from these models increases our understanding of (complex) phenomena and consequently enables us to exercise control over such phenomena. For instance, knowledge concerning medical conditions enables us to better control the condition through treatment, prevention, etc. In general, knowledge is acquired by distinguishing components of a phenomenon and then investigating the function of each component. In time, the parts are reintegrated in order to gain insight regarding the whole of the phenomenon. This strategy has been employed throughout history and is still employed whenever we are confronted with phenomena we cannot (fully) explain or comprehend.

Pain is a specific example of a phenomenon that human beings are often confronted with and would like to control. In particular, long-lasting pain that lacks a clear physical cause (chronic benign pain) is a phenomenon that is not yet fully understood and raises many questions about its origin and treatability. In 2000 , approximately $15 \%$ of the general population in the Netherlands suffered from chronic a-specific low back pain. This prevalence is expected to increase by another $14 \%$ before 2020 (RIVM). Chronic benign pain is clearly a highly prevalent phenomenon that not only inconveniences those subjected to the pain but also substantially burdens health care systems. Treatment for low back pain and for chronic pain in general is often non-optimal (Mes, 2007). Although the average outcome of treatment programs is often positive, previous research has demonstrated a large variability around this average (Mes, 2007). This essentially means that, although some patients are treated successfully, other patients experience no improvement and, in some cases, patients even get worse, e.g. report an increase in pain. All kinds of pain-treatments can be considered, at least in part, 'non-optimal' because knowledge regarding etiologic, pain persistent and treatment mechanisms of chronic pain is still limited. The complex co-morbidity surrounding pain (Tunks et al, 2008, Von Korff et al, 2005) in addition to the multidimensional nature of pain (Melzak, 1999) and difficulties with respect to pain measurement, likely contribute to this lack of knowledge. Before going into detail on these factors, the historical background and definitions of pain will first be discussed.

Since ancient time, scientists have attempted to explain the mysteries of pain. Culture and time seem to determine how pain is defined. In ancient Egypt and India, pain without physical damage was considered to be caused by gods and spirits as punishment for human folly (Bonica, 1990). In the seventeenth century, Descartes (1664) argued that pain was directly related to an external noxious stimulus. Pain signals were transmitted to the brain via a fixed pathway of nerves which looked like tubes consisting of a large number of threads. Descartes' conception of pain was the precursor to the first pain theory formulated, namely the specificity theory. The specificity theory posited that pain is a specific sensation that is separate from touch 
and has its own apparatus. The specificity theory further assumed that pain is proportional to physical damage. Although this theory seemed plausible, it failed to explain both clinical observations of pain in the absence of noxious stimuli and the absence of pain despite severe injury (Beecher, 1946). As a result, the paradigm surrounding pain shifted from a purely physical explanation of pain to a predominantly psychological explanation (e.g. operant conditioning (Fordyce et al., 1976) and social learning theory (Craig, 1983)). However, these theories and their corresponding unidimensional models were found to inadequately explain all circumstances in which pain can occur. Consequently, by the mid-sixties, the first multidimensional model of pain, namely the Gate Control Theory (GCT), was formulated. This theory, described by Melzack and Wall (1965), states that pain is the result of an interaction between three systems (dimensions) that are active in the processing of nociceptive stimuli: a) the sensory-discriminative system which reflects the processing of nociceptive stimuli under normal circumstances; b) the motivationalaffective system which concerns the affective response as a direct reaction to the stimulus; and c) the cognitive-evaluative system which concerns the emotional discomfort that is caused by the pain. Additionally, the GCT postulates a gate mechanism in the dorsal horns of the spinal cord which regulates the passage of nociceptive signals from peripheral nerves to the central nervous system (CNS). The opening and closing of the gate is hypothesized to be influenced by ascending peripheral nerves and descending nerves from the CNS. Through these descending nerves from the CNS, cognitive and psychological factors can influence pain.

Throughout the years, brain research has attempted to map brain areas and pathways involved in the processing of pain. These different structures are depicted in Figure 1 and include the anterior cingulate cortex, the amygdala, the thalamic nuclei, the hippocampus, the primary and secondary cortices, the primary and secondary motor cortices, the prefrontal and posterior parietal cortices, the basal ganglia, the periaqueductal grey matter and the cerebellum. These brain areas are often collectively referred to as the 'pain matrix' (Willis et al., 1997; Peyron et al, 2000; Apkarian et al, 2006). Connecting lines between the different brain areas indicate that the corresponding areas influence one another. These brain regions are also activated in response to non-noxious stimuli, thus suggesting that a specific pain centre in the brain does not exist. 


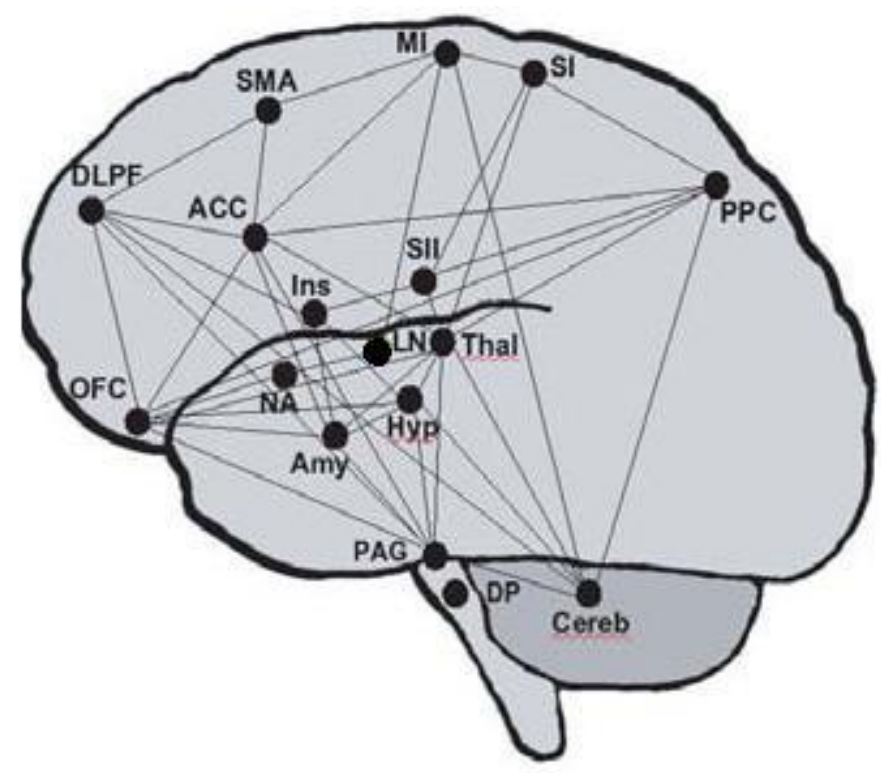

Figure 1. The Pain Matrix adopted from Kuper \& Kehlet (2006). SI=primary somatosensory cortex. SII=secondary somatosensory cortex. PPC=posterior partial cortex. DLPF=dorsolateral prefrontal cortex. OFC=orbitofrontal cortex.

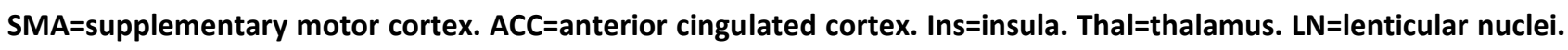
NA=nucleus accumbens. Amy=amygdale. Hyp=hypothalamus. PAG=periaquaductal grey. DP=dorsal pons. Cereb=cerebellum. $\mathrm{MI}=$ primary motor cortex.

The theoretical foundation of most pain research today is still rooted in the GCT thus reflecting acceptance of multidimensionality in the study of pain. In fact, the definition provided by the International Association of the Study of Pain (IASP, 1979) is as follows: "Pain is an unpleasant sensory and emotional experience associated with actual or potential tissue damage, or described in terms of such damage", Clearly, pain is not considered to be merely influenced by physical damage but also by psychological, cognitive and environmental factors (Figure 2). Pain research conducted in the last three to four decades has largely been focused on demonstrating this multidimensionality. Studies have found that depression and anxiety influences the persistence of pain (Linton, 2000; Van Korff \& Miglioretti, 2005). Furthermore, cognitive and environmental factors have been demonstrated to influence pain (McBeth et al, 2003; Picavet et al, 2002). 


\section{OUTSIDE ENVIRONMENT}

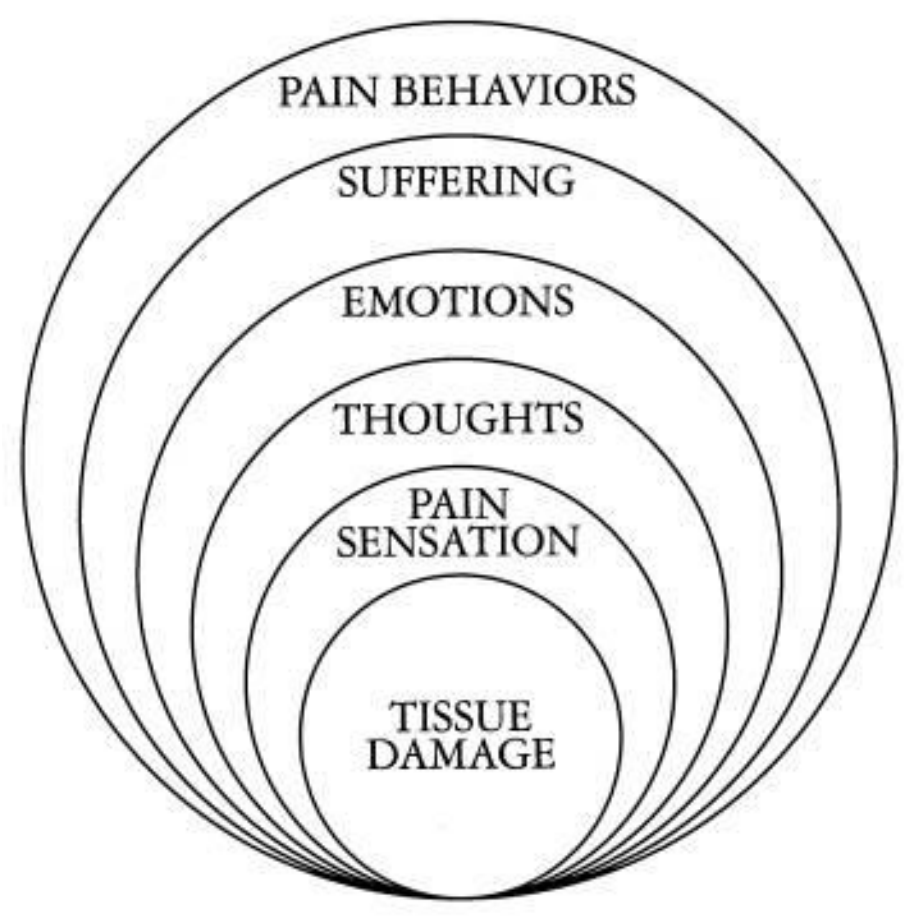

Figure 2. Loesers "onion" model of pain illustrating the multidimensionality of pain. Adopted from Deardorff (2004).

Pain research, is restricted by a fundamental problem, namely that pain is a subjective experience. Because of this subjectivity, pain cannot directly be measured. Strictly speaking, only pain derivatives can be measured. Measurement is further complicated by the multidimensional character of pain as most instruments only measure a single aspect of pain. Perhaps the most frequently used instruments are the Visual Analogue Scale (VAS) and the Numeric Rating Scale (NRS). These subjective measures assess the amount of pain but are sensitive to various kinds of bias including memory bias (Magnusson et al, 1995) and experimenter bias (Branch et al., 2000).

In an attempt to measure relatively unbiased pain responses, psychophysiological measures such as skin conductance (Peters \& Schmidt, 1991), heart rate (Lindh et al., 1997), electromyography (Collins et al., 1982) and finger pulse volume (Arntz \& Lousberg, 1990) have been employed. In recent decades, a timelocked derivative of the electroencephalogram termed the Event-Related Potential (ERP) has also been used as a psychophysiological measure of pain. In contrast to the aforementioned psychophysiological measures, specific ERP components have been found to correlate relatively high with subjective pain estimates. Studies report correlations between specific peak amplitudes and subjective pain ratings of 0.4 to 0.5 (Miltner, 1989; Bromm et al, 1998). The ERP is considered to represent the averaged cortical processing of a 
specific stimulus (e.g. pain stimulus). As such, the ERP is a summary measure of all processes that are involved in the response to a pain stimulus. However, the spatial resolution of the ERP is considered low because the electrical responses are smeared over the scalp and thus the localisation of the generator is difficult (Devinsky \& D'Esposito, 2004). More recently developed brain imaging techniques such as PET or fMRI are superior in that area. Nonetheless, the ERP provides the most direct measure of neuronal activity (Kupers \& Kehlet, 2006) because the temporal resolution is especially high. Processes that are active in response to a pain stimulus can be measured directly within milliseconds. In other words, the ERP is a measure of function whereas fMRI and PET are measures of brain structures. Therefore, the ERP seems to be a promising objective measure of pain when investigating pain mechanisms. There are, however, several questions about the interpretation and the (statistical) handling of Event-Related Potentials. The following chapters of this dissertation will address these questions.

\section{This dissertation}

In the context of the theoretical background and framework described above, this dissertation endeavours to increase our understanding of chronic pain through a series of studies employing the promising technique of ERP as an objective measure of pain. Five specific questions regarding the use and the implications of the ERP technique were formulated: 1) How can ERP best be handled in a statistical manner when we want to obtain (detailed) information about pain mechanisms?; 2) How do amplitudes of painERPs relate to subjective ratings of pain and is this relationship influenced by factors such as stimulus intensity and habituation?; 3) Can we use pain-ERPs obtained in the laboratory to predict clinical pain?; 4) Is there a difference in habituation of cortical pain processing between chronic pain patients and pain-free control subjects?; and 5) What role do gene polymorphisms play in the cortical processing of pain?

\section{Preliminary findings}

Chapter 2 describes a study that functioned as a precursor of the studies described in the later chapters. The goal of this study was twofold. It firstly sought to demonstrate the multidimensionality of pain by showing the psychological factor neuroticism moderates the cortical processing of pain. Secondly, it endeavoured to show that neuroticism was not, as often thought, a homogeneous but rather a heterogeneous concept. Based on findings from previous studies, we hypothesized that two subfacets of neuroticism, namely anxiety and depression, have a dissimilar moderating effect on the pain-ERP. Studies investigating the influence of anxiety on pain are straightforward in stating that anxiety increases pain 
responses (Gibson et al., 1991; Munafo \& Stevenson, 2001; Tang et al., 2005). The results of studies on depression and pain are inconsistent, reporting in some cases, that pain augments depression, and in others, that pain reduces the effects of depression (Hansenne et al., 2000; Lépine \& Briley, 2004; McWilliams et al., 2004). Consequently, the way in which these subfacets of neuroticism influence the cortical processing of pain was investigated independently in this preliminary study.

\section{Statistical handling of ERP data}

The ERP technique, often used in pain research, is considered to be a more objective measure compared to subjective ratings. Since its inception, many developments regarding how ERP data should be been handled, have taken place. Of these, most have focused on how ERP is recorded and how the data can best be processed. Little attention, however, has been paid to how ERP data can best be handled statistically. The traditional statistical approach, which is still frequently used in current ERP research, entails averaging trials and performing t-tests or analyses of variance (ANOVAs) to test for statistical differences between groups. Unfortunately, there are difficulties with this approach (Hoormann et al, 1998; Nikulin et al, 2007; Mazaheri \& Jensen, 2008). Chapter 3 outlines these difficulties and proposes a new approach to the statistical handling of pain-ERP data. The aim of this study was to demonstrate the advantages of using multilevel analysis in ERP research and to demonstrate that this tool is not only appropriate but even necessary in fundamental research on pain.

\section{The relationship between pain-ERPs and subjective ratings of experimental versus clinical pain}

As stated above, ERPs are considered to be good correlates of the pain experience. In order to apply this knowledge clinically, we need to understand the relationship between pain-ERPs and the subjective experience of pain. Previous research has demonstrated positive correlations between the amplitude of specific peak components and the subjective experience of pain (Harkins \& Chapman, 1978; Chen et al, 1979; Garcia-Larrea et al, 1997). In chapter 4, we re-examined the association between ERPs and the subjective experience of pain in a more comprehensive manner. Using multilevel analyses, the relationship between ERP and subjective pain ratings was studied while taking into account specific confounders such as intensity and habituation.

An additional and important issue is the transferability of experimental findings to daily life. As such, it is important that we understand how the cortical processing of experimental pain measured in laboratories relates to chronic pain in daily life. Can we root our assumptions about chronic pain in ERP data and can this 
data be used to predict chronic pain? Evidently, in order to apply the findings of ERP data in clinical practice, it is imperative that we understand how pain-ERPs relate to clinical pain. Similar investigations have been conducted previously in research on depression but not in pain research. Consequently, chapter 5 investigates whether ERPs obtained in the laboratory predict pain ratings completed by participants in the two weeks following the experiment.

\section{Differences in cortical pain processing between chronic low back pain patients and pain-free control subjects}

Low back pain is a frequently reported pain complaint. In most cases, low back pain disappears spontaneously within one or two weeks. However, in approximately $10-20 \%$ of the cases, the pain persists in the absence of a clear physical cause (Bekkering et al., 2003). An important question in pain research is: Why do some people develop chronic low back pain while most others do not? What is the difference between chronic pain patients and pain-free control subjects? Previous research has frequently attempted to find a mechanism that explains why some people develop chronic pain after an acute injury and others do not. A deficit in habituation to painful stimuli has been proposed as an explanation for the chronification of pain. However, only a limited number of studies have actually investigated whether chronic pain patients do indeed have a greater deficit in habituation than in comparison to pain-free control subjects (Brandt \& Schmidt, 1987; Peters et al., 1989; Arntz et al, 1990; Valeriani et al, 2003; Tommaso et al., 2005). Chapter 6 describes a study that endeavoured to comprehensively examine differences in habituation of experimental pain stimuli between chronic pain patients and pain-free control subjects. More specifically, this study investigated whether chronic pain patients had reduced habituation compared to pain-free control subjects via multilevel analyses with ERPs as the outcome measure. Furthermore, since previous literature has demonstrated that depression and pain are related and that depression can an influence pain-ERPs (Tang et al., 2008; Young et al., 2008), the moderating influence of depression on differences in habituation was also investigated.

Genetic factors may also play a role in inter-individual differences in pain and, as such, may in part explain why some people develop chronic pain while most others do not. Previous research has proposed specific 'pain gene candidates', namely COMT Val158Met, BDNF Val66Met and OPRM1 A118G (Šerý et al., 2005; Max et al., 2006; Buskila, 2007). Unfortunately, experimental research concerning these polymorphisms is scarce. Consequently, the aim of the study discussed in chapter 7 was to investigate the influence of these polymorphisms on the cortical processing of pain. In this study, we explored whether the possible influence of polymorphisms differed between chronic pain patients and pain-free control subjects. 


\section{References}

Apkarian AV, Bushnell MC, Treede RD, Zubieta JK (2005) Human brain mechanisms of pain perception and regulation in health and disease. European Journal of Pain, 9, 463-84.

Arntz A, Lousberg R (1990) The effects of underestimated pain and their relationship to habituation. Behavioural Research Therapy, 28, 15-28.

Bekkering GE, Hendriks HJM, Koes BW, Oostendorp RAB, Ostelo RWJG Thomassen JMC van Tulder MW (2003) KNGFguidelines for physical therapy in patients with low back pain. Nederlands Tijdschrift voor Fysiotherapie, 111. https://www.cebp.nl/media/m163.pdf

Bonica JJ (1990) History of pain concepts and therapies. In The Management of Pain (Ed. Bonica JJ), pp. 3-17. Lea \& Febiger: Philadelphia.

Branch MA, Carlson CR, Okeson JP (2000) Influence of biased clinician statements on patient report of referred pain. Journal of Orofacial Pain, 14, 120-127.

Buskila D (2007) Genetics of chronic pain states. Best Practice \& Research Clinical Rheumatology, 21, 535-547.

Chen CAN, Chapman CR, Harkins SW (1979) Brain potentials are functional correlates of induced pain in man. Pain, 6, 365-374.

Collins GA, Cohen MJ, Naliboff BD, Schandler SL (1982) Comparative analysis of paraspinal and frontalis EMG, heart rate and skin conductance in chronic low back pain patients and normals to various postures and stress. Standard Journal of Rehabilitation Medicine, 14, 39-46.

Craig KD (1983) Modeling and social learning factors in chronic pain. Advances in Pain Research and Therapy, 5, 813827.

Devinsky O, D’Esposito M (2004) Neurology of cognitive and behavioural disorders. Oxford University Press.

Fordyce WE, Fowler RS, Lehmann JF, DeLateur BJ, Sand PL, Trieschmann RB (1973) Operant conditioning in the treatment of chronic pain. Archives of Physical Medicine and Rehabilitation, 54, 339-408.

Garcia-Larrea L, Peyron R, Laurent B, Mauguiere F (1997) Association and dissociation between laser-evoke potentials and pain perception. Neuroreport, 8, 3785-3789.

Gibson SJ, LeVasseur SA, Helme RD (1991) Cerebral event-related responses induced by $\mathrm{CO}_{2}$ laser stimulation in subjects suffering from cervico-brachial syndrome. Pain, 47, 173-182.

Hansenne M, Pitchot W, Pinto E, Reggers J, Papart P, Ansseau M (2000) P300 event-related brain potentials and personality in depression. European Psychiatry, 15, 370-377.

Harkins SW, Chapman CR (1978) Cerebral evoked responses to noxious dental stimulation: relationship to subjective pain report. Psychophysiology, 15, 248-252.

Hoormann J, Falkenstein M, Schwarzenau P, Hohnsbein J (1998) Methods for the quantification and statistical testing of ERP differences across conditions. Behavioural Research Methods Instruments \& Computers, 30, 103-109.

Horn S, Munafó M (1997) Pain: Theory, research and interventions. Open University Press: Buckingham.

International Association for the Study of Pain (1979) Pain, 6, 250. 
Jeffrey JA (2005) Chronic pain. In Chronic Illness: impact and interventions (Eds. Lubkin IM \& Larson PD), pp. 67-104. Jones \& Bartlett Publishers Inc.: Boston.

Kupers R, Kehlet, H (2006) Brain imaging of clinical pain states: a critical review and strategies for future research. Lancet Neurology, 5, 1033-1044.

Lépine JP, Briley M (2004) The epidemiology of pain in depression. Human Psychopharmacology: Clinical and Experimental, 19, S3-S7.

Lindh V, Wiklund U, Sandman PO, Håkansson S (1997) Assessment of acute pain in preterm infants by evaluation of facial expression and frequency domain analysis of heart rate variability. Early Human Development, 48, 131-142.

Linton SJ (2000) A review of psychological risk factors in back and neck pain. Spine, 25, 1148-1156.

Magnusson T, List T, Helkimo M (1995) Self-assessment of pain and discomfort in patients with temporomandibular disorders: a comparison of five different scales with respect to their precision and sensitivity as well as their capacity to register memory of pain and discomfort. Journal of Oral Rehabilitation, 22, 549-556.

Manchikanti L, Singh V, Datta S, Cohen SP, Hirsch A (2009) Comprehensive Review of Epidemiology, Scope and Impact of Spinal Pain. Pain Physician, 12, E35-70.

Max MB, Wu T, Atlas SJ, Edwards RR, Haythornthwaite JA, Bollettino AF, Hipp HS, McKnight CD, Osman IA, Crawford EN, Pao M, Nejim J, Kingman A, Aisen DC, Scully MA, Keller RB, Goldman D, Belfer I (2006) A clinical genetic method to identify mechanisms by which pain causes depression and anxiety. Molecular Pain, 2, 14-27.

Mazaheri A, Jensen O (2008) Asymmetric Amplitude Modulations of Brain Oscillations Generate Slow Evoked Responses. The Journal of Neuroscience, 28, 7781-7787.

McBeth J, Harkeness EF, Silman AJ, Macfarlane GJ (2003) The role of workplace low level trauma, posture and environment in the onset of chronic widespread pain. Rheumatology, 42, 1486-1494.

McWilliams LA, Goodwin RD, Cox BJ (2004) Depression and anxiety associated with three pain conditions: results from a nationally representative sample. Pain, 111, 77-83.

Melzack R, Wall P (1986) The Challenge of Pain. Pinguin Books Ltd.: Harmondsworth.

Melzack R (1999) Pain: an overview. Acta Anaesthesiologica Scandinavica, 43,880-884.

Mes, C (2007) Improving non-optimal results in chronic pain treatment: A tripartite approach. Dissertation. Gildeprint Drukkerijen: Enschede, the Netherlands.

Nash TP (2005) Editorial II: What use is Pain? Britisch Journal of Anaesthesia, 94, 146-149.

Nikulin VV, Linkenkaer-Hansen K, Nolte G, Lemm S, Müller KR, Ilmoniemi RJ, Curio G (2007) A novel mechanism for evoked responses in the human brain. European Journal of Neuroscience, 25, 3146-3154.

Peters ML, Schmidt AJ (1991) Psychophysiological response to repeated acute pain stimulation in chronic low back pain patients. Journal of Psychosomatic Research, 35, 59-74.

Peyron R, Laurent B, Garcia-Larrea L (2000) Functional imaging of brain responses to pain: a review and meta-analysis. Neurophysiologie Clinique, 30, 263-88. 
Picavet HS, Vlayen JW, Schouten JS (2002) Pain catastrophizing and kinesiophobia: predictors of chronic low back pain. American Journal of Epidemiology., 156, 1028-1034.

Šerý O, Hrazdilová O, Matalová E, Ševčik P (2005) Pain research update from a genetic point of view. Pain Practice, 5, 341-348.

Tang J, Gibson SJ (2005) A Psychophysical Evaluation of the Relationship Between Trait Anxiety, Pain Perception, and Induced State Anxiety. Journal of Pain, 6, 612-619.

Tang NKY, Salkovskis PM, Hodges A, Wright KJ, Magdi H, Hester J (2008) Effects of mood on pain responses and pain tolerance: An experimental study in chronic back pain patients. Pain, 138, 392-401.

Tunks ER, Crook J, Weir R (2008) Epidemiology of chronic pain with psychological comorbidity: prevalence, risk, course and prognosis. Canadian Journal of Psychiatry, 53, 224-234.

Turk DC, Flor H (1984) Etiological Theories and Treatments for Chronic Back Pain. II. Psychological models and interventions. Pain, 19, 209-233.

Von Korff M, Crane P, Lane M, Miglioretti DL, Simon G, Saunders K, Stang P, Brandenburg N, Kessler R (2005) Chronic spinal pain and physical-mental comorbidity in the United States: results from the national comorbidity survey replication. Pain, 113, 331-339.

Von Korff, Miglioretti DL (2205) A prognostic approach to define chronic pain. Pain, 117, 304-313.

Willis WD, Westlund KN (1997) Neuroanatomy of the pain system and of the pathways that modulate pain. Journal of Clinical Neurophysiology, 14, 2-31.

Young CC, Greenberg MA, Nicassio PM, Harpin E, Hubbard D (2008) Transition from acute to chronic pain and disability: a model including cognitive, affective and trauma factors. Pain, 134, 69-79. 



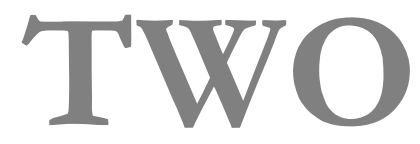

\section{Evidence that Trait-Anxiety and Trait-Depression Differentially}

\section{Moderate Cortical Processing of Pain}

Reference

Vossen HGM, van Os J, Hermens H, Lousberg R (2006) Evidence that Trait-Anxiety and Trait-Depression differentially moderate cortical processing of pain. Clinical Journal of pain, 22, 725-729. 


\section{Abstract}

The purpose of this study was to examine the influence of neuroticism $(\mathrm{N})$ and its different subfacets anxiety ( $\mathrm{N}$-anx) and depression ( $\mathrm{N}$-dep) on cortical pain processing expressed as event-related potentials (ERPs). Seventy-five healthy subjects received electrical pain stimuli under condition of simultaneous EEG registration. Multiple regression analyses were conducted to study the amount of pain-ERP variance. ERP variance was explained by neuroticism and its subfacets. Neuroticism (N) moderated the way pain was processed cortically. Higher levels of $\mathrm{N}$ were associated with higher pain-ERP amplitudes. Within this association, differential sub-facet effects were observed: $\mathrm{N}$-anx reduced, whereas $\mathrm{N}$-dep augmented pain-ERP amplitudes. A personality trait reflecting bias towards negative emotions may moderate the way pain is processed cortically, with directionally different effects depending on whether the trait is expressed predominantly in the realm of anxiety or depression. 


\section{Introduction}

Pain is a frequent and multidimensional phenomenon that often runs a chronic course. Despite extensive research, many questions remain. Pain is not merely a physical experience, as it is also moderated by psychosocial, cognitive and cultural factors (Melzack \& Wall, 1965). How these factors interact together to give rise to the conscious appraisal of pain, is unclear and likely complex. More insight into the basic causal mechanisms of (chronic) pain is crucial in order to develop more effective treatments.

Some of the most important moderators are in the realm of personality, in particular the personality trait referred to as neuroticism (N). $\mathrm{N}$ is defined as a tendency to experience negative emotions in stressful situations (Costa \& McCrae, 1985). Studies have consistently reported positive associations between $\mathrm{N}$ and the experience of pain (Costa \& McCrae, 19820; Wade et al, 1992). However, different and, at times, opposing explanations have been proposed for the underlying causal mechanism of this association (BenDebba et al, 1997; Johnson, 2003).

The relation between $\mathrm{N}$ and pain is often explained in terms of over-reporting of pain-related complaints and an exaggerated expression of disturbance. Groth-Marnat and Fletcher (2000) suggest that persons with high levels of $\mathrm{N}$ tend to be more focused on their bodily states than others and thus report more physical complaints. According to Costa and McCrae (1985), physical complaints of persons high in $\mathrm{N}$ are best viewed as exaggerations of bodily concerns, linking neuroticism to hypochondria. In contrast, Johnson (2003) suggests that rather than prone to over-reporting or exaggeration, individuals high in $\mathrm{N}$ are truly more vulnerable to physical symptoms, whereas Harkins and colleagues (1989) propose that $\mathrm{N}$ impacts on the cognitive appraisals associated with the processing of pain stimuli such as meaning and implications. A further observation is that those scoring high on $\mathrm{N}$ perceive experimental pain as well as chronic pain as more disturbing than others (Harkins et al, 1989). This latter finding suggests that $\mathrm{N}$ does not moderate the pain intensity perceived, but rather that $\mathrm{N}$ is associated with the immediate unpleasantness induced by pain (Harkins et al, 1989; Wade et al, 1992).

The conflicting explanations revolving around $\mathrm{N}$ and the experience of pain may in part be the result of methodological and conceptual difficulties. Pain research is restricted by a fundamental problem. Pain is a subjective experience and therefore direct measurement is not possible. As a more objective alternative for bias-prone self-report questionnaires, Event (pain) Related Potentials (ERP) are increasingly employed. This psychophysiological measure represents time-locked cortical processing. Relatively strong associations $(r=0.4-0.5)$ have been reported between ERP peak amplitudes and 
subjective ratings of pain (Miltner et al, 1989). However, despite these encouraging findings, ERPs have rarely been used in elucidating the relationship between pain and personality.

A further issue is that neuroticism is generally treated as a homogeneous concept. However, factoranalytic approaches suggest that $\mathrm{N}$ represents a heterogeneous set of traits. The NEO big five Personality Inventory distinguishes 6 facets of $\mathrm{N}$, namely anxiety, impulsivity, depression, selfconsciousness, irritability and vulnerability. Despite their separate delineation, these subfacets of $\mathrm{N}$ have rarely been studied separately in relation to pain. However, some of the subfacets of $\mathrm{N}$ have been studied as a clinical state rather than a trait. Many studies have demonstrated that (chronic) pain can be influenced by depression and anxiety as a (clinical) state (Keogh et al, 2004; Feeney, 2004). Unfortunately, the manner in which pain is influenced by these concepts remains unclear. With respect to anxiety research, the results are mixed. Anxiety can be divided in state anxiety and trait anxiety. The general consensus is that state anxiety increases pain sensitivity. Anxiety related to pain, such as preoperative anxiety, seems to have an augmenting effect (Munafo \& Stevenson, 2001). However, the results on trait anxiety (often measured with the State-Trait Anxiety Inventory), are mixed. Arnzt and colleagues (1993) suggest that trait anxiety which is not related to pain has an attention diverting effect and thus reduces pain sensitivity. In contrast, Tang et al (2005) found that trait anxiety similar to state anxiety augments pain sensitivity. In addition, information, albeit limited, on the relationship between state anxiety and pain Evoked Potentials is available. The results of a study by Gibson and colleagues (1991) show that state anxiety is associated with relatively high pain-ERP amplitudes. Results from studies regarding the association between depression and pain are ambiguous. Many studies report a positive association between depression and pain (Lépine \& Briley, 2004). This association is mainly reflected in the high incidence of co-morbid depressive complaints in chronic pain populations (McWilliams et al, 2004). On the other hand, a pain-reducing effect of depression has also been reported (Hansenne et al, 2000).

A study was designed in which the two facets of $\mathrm{N}$ that were a priori hypothesized to act as pain moderators, namely depression and anxiety, were studied independently of each other in relation to cortical processing of pain, using the objectifying approach of ERP. The following hypotheses were tested: i) Pain-ERP amplitudes are positively associated with $\mathrm{N}$; and ii) within $\mathrm{N}$, the subfacets of trait anxiety and trait depression are independently associated with pain-ERP amplitudes. Because of the above-mentioned ambiguity of the influence of depression and anxiety on pain, directional hypotheses were not formulated. 


\section{Materials and Methods}

\section{Participants}

Seventy-five healthy subjects ( 41 women and 34 men) participated, ranging in age from 18 to 58 years (mean 24.5, SD=6.39). Most of the subjects were students. Participants were pain-free subjects, had no history of chronic pain complaints and did not use analgesics or psychoactive medication. Subjects participated voluntarily and signed an informed consent approved by the standing ethics committee. Participation was rewarded with $€ 15$.

\section{Stimulus materials}

The stimuli used were electrical shocks of 10 milliseconds duration, administered by intracutaneous finger stimulation (Bromm \& Meier, 1984). Five different intensities, based on the subject's selfdetermined pain threshold, were presented. The two lowest intensities were $50 \%$ and $25 \%$ beneath the pain threshold (respectively named level 1 and 2) and the two highest intensities $25 \%$ and $50 \%$ above the pain threshold (level 4 and 5). The final intensity level was equal to the pain threshold (level 3 ). In this study, 150 shocks (30 per intensity) were presented with inter-stimulus intervals ranging from 9 to 11 seconds.

\section{Experimental task}

All subjects received the rating paradigm developed by Bromm \& Meier (1984). This paradigm consists of semi-randomly presented electrical shocks. Subjects were asked to rate the intensity of every shock on a scale from 0 to 100, zero being no sensation and 100 being the most excruciating pain imaginable.

\section{Procedure}

Before starting the experiment, subjects were informed about the purpose of the study. Subjects were told that EEG would be measured while they received various intensities of electric shocks - some painless, some painful. After signing an informed consent, the electrodes for the EEG were placed. The following step was the attachment of the shock electrode to the top of the left middle finger. Using this method, described by Bromm and Meier (1984), a small opening in the upper layer of the skin was prepared using a dental gimlet. Care was taken that this procedure was not painful. In the prepared 
opening, a golden electrode was placed and fixed with tape. Two copper laces were attached around the prepared finger and wrist. Next, the pain threshold was determined by gradually increasing the stimulus intensity, starting with zero intensity. Subjects were asked to say "stop" as soon as they perceived the intensity of the stimulus as painful. After the pain threshold was determined, the rating paradigm was started.

\section{Psychological Assessment}

After signing the informed consent form, all subjects were asked to complete the NEO Personality Inventory. The NEO-PI-R is based on a five-factor model of personality. The five personality factors measured are Neuroticism, Extraversion, Openness, Agreeableness and Conscientiousness. All these factors are divided in six subfacets. The subfacets of $\mathrm{N}$ are anxiety, hostility, depression, selfconsciousness, impulsiveness and vulnerability. The $\mathrm{N}$ subfacets anxiety and depression were used for the statistical analyses (hereafter: $\mathrm{N}$-anx and $\mathrm{N}$-dep, respectively). Validity and reliability measures of the NEO-PI-R have proven to be adequate (Hoestra et al, 1996).

\section{EEG recording}

All EEG recordings were conducted in an electrically- and sound-shielded cubicle $\left(3 * 4 \mathrm{~m}^{2}\right)$. Three $\mathrm{Ag} / \mathrm{AgCl}$ electrodes were placed on $\mathrm{Fz}, \mathrm{Cz}$ and $\mathrm{Pz}$ using the international 10-20 system (Jasper, 1958). Impedances were kept below $5 \mathrm{k} \Omega$. A reference electrode was placed on the right ear lobe. To control for possible vertical eye movements, an electro-oculogram (EOG) electrode was placed 1 centimeter under the midline of the right eye. A ground electrode was placed at Fpz. All electrodes were fixed using Elefix pasta. Neuroscan 4.2 software was used for EEG recording.

\section{Data Reduction and Statistical Analysis}

Trials with EOG artefacts (amplitudes greater than $+/-100 \mu \mathrm{V}$ ) were excluded from analyses. When more than $50 \%$ of all trials within a stimulus intensity category had to be excluded due to EOG artefacts, the participant was entirely excluded from the analyses. Almost all analyses were conducted using the statistical computer program SPSS 11.0. The multilevel analyses were conducted using STATA 8.

Because of the complex structure of the averaged (pain) ERP, a new, more appropriate way of analyzing pain-ERPs, developed by Lousberg and colleagues (unpublished data) was used. According to this method, the ERP amplitude at any given time point (latency) is considered to be a function of 
measurement/technical variables, paradigm variables, and variables related to neurophysiological, (neuro)psychological and cultural characteristics within the subject. Since the dependent variable, the ERP amplitude, is expected to be normally distributed, linear regression analysis were used to explain the variability at each point of latency. As a tradeoff between an infinite number of analyses and a loss of view on the course of effects, intervals of 10 milliseconds were used. These regression analyses were performed simultaneously. Due to the fact that the morphology of pain and non-pain-ERPs are highly similar, non-pain amplitudes (levels 1 and 2) were included in the regression model. In this manner, nonpain related information can be subtracted from the pain-ERP. With the resulting 'pain'ERP information the hypotheses can be tested. Since a large number of regression analyses will be performed, the chance of finding significant results is increased. Therefore we decided to interpret only robust effects by selecting effects that remain significant during at least 50 milliseconds. For each regression analysis, outlying cases were identified with the formula $4 /(n-k-1)$ and removed (Hair et al, 1998). All reported pvalues were two tailed and assumptions of normality and linearity were checked studying regression residuals. In addition, because of the inter-individual differences in absolute stimulus intensity level, this variable was included as a covariate in the regression analyses. Thus, all analyses were performed with inclusion of stimulus intensity level as a covariate. Finally, multilevel analyses were performed to examine associations between personality and the subjective pain ratings, in order to additionally take into account that observations were nested within persons, which could compromise their statistical independence.

\section{Results}

Of the 75 subjects, 17 were excluded due to excessive eye movements or technical problems resulting in 58 analyzable cases. This group consisted of 28 men (48\%) and 30 women (52\%). With respect to the absolute stimulus intensity level, almost identical result were found with and without this variable included in the regression model.

\section{The effect of $\mathbf{N}$ on the pain-ERP}

First, pain-ERP curves for low and high $\mathrm{N}$ subgroups were created with the median split procedure (see Figure 1a). Visual inspection indicated more positive ERP amplitudes for the high $\mathrm{N}$ group during a broad latency range. Regression analyses were carried out, with ERP amplitude (level 5) as the dependent variable and $\mathrm{N}$ score (as a continuous variable), the non-pain-ERP amplitudes (level 1 and 2 ) and 
stimulus intensity level as independent variables. These analyses confirmed the visual observations. Significant $N$ effects on the pain-ERP amplitude were observed between 340-400 milliseconds, 730-860 milliseconds (exclusively on Fz) and 1240-1450 milliseconds. In these latency ranges, this amplitude effect indicated stronger cortical pain processing for the higher $\mathrm{N}$ scores, thus supporting hypothesis 1.

\section{Independent cortical effects of trait anxiety and trait depression}

To study the independent influence of $\mathrm{N}$-anx and $\mathrm{N}$-dep on the pain-ERP, another series of regression analyses was carried out. Again, the ERP amplitude of the highest intensity (level 5) was the dependent variable, and the predictors were the non-pain-ERP amplitudes (level 1 and 2), N-anx and N-dep scores and the absolute stimulus intensity level. In Figure $1 \mathrm{~b}$, the t-values of the regression coefficients of the $\mathrm{N}$-anx and $\mathrm{N}$-dep parameters are presented. The following observations can be made from this figure. First, there are several latency ranges with significant independent effects of $\mathrm{N}$-anx and $\mathrm{N}$-dep on the pain-ERP amplitude. Second, the effects of $\mathrm{N}$-anx and $\mathrm{N}$-dep were directionally dissimilar, with $\mathrm{N}$-dep showing an amplitude augmenting effect and $\mathrm{N}$-anx an amplitude reducing effect, with the exception of $\mathrm{N}$-anx having an amplitude augmenting effect in the latency range 340-410 milliseconds. These results support hypothesis 2 .

\section{Effects of Neuroticism on subjective ratings of pain}

To give experiential meaning to the psychophysiological results found above, additional analyses regarding the subjective ratings of pain were performed with the subjective ratings (VAS-scores) of the highest intensity as dependent variable and the $\mathrm{N}$-scores as the independent variable. Unexpectedly, results did not show a significant effect $(Z=-1.15, p=0.249)$ between the level of neuroticism and the subjective ratings to pain. Similar results were found for the subfacets $\mathrm{N}$-anx $(\mathrm{Z}=0.54, \mathrm{p}=0.592)$ and $\mathrm{N}$ $\operatorname{dep}(Z=1.37, p=0.169)$.

\section{Discussion}

The aim of this study was to clarify the relationship between neuroticism (N) and pain. The use of EventRelated Potentials in relation to a phenotype such as pain requires caution in interpreting the results, given the lack of a full understanding of the underlying physiology driving ERP, its relationship to conscious awareness, and the experimental nature of the pain measures used in this research. Although 
earlier research has investigated the meaning of specific peaks, it is difficult to use this knowledge since this study does not focus on peak amplitudes.

While acknowledging these fundamental limitations, it was demonstrated that persons with higher scores on the $\mathrm{N}$-scale showed differential cortical processing of painful electrical stimuli in comparison to persons with lower scores. Effects were not only restricted to the P300 range (340-400 milliseconds) but were also evident at much later latency points (730-860 milliseconds and 1240-1450 milliseconds). At these latency ranges, individuals high in $\mathrm{N}$ showed more positive ERP amplitudes, thus suggesting stronger pain processing. Becker and co-workers (1993) state that a positive wave that peaks around 330 milliseconds reflects a neural response difference between strong non-painful stimuli and painful stimuli. This positive wave is a specific pain component. In addition, Lousberg and colleagues (unpublished data) showed that intensity was significantly embedded in a much broader latency range (200 to 1500 milliseconds) of the pain-ERP. This would suggest that stronger amplitudes for persons high in $\mathrm{N}$ in later latency ranges are also correlated with the intensity of the stimulus.

These findings cannot be readily translated to an experiential level. Analyses with subjective pain ratings did not show significant differences in subjective pain experience between persons with low and high $\mathrm{N}$ scores. An explanation for this could be the fact that the stimulus intensities were normalized for each subject. The first stimulus (threshold level) was set at a VAS score of 60. It could be argued that this reduces the variability of the VAS responses. However, our own observation during the experiment was that there was a broad range of VAS responses per stimulus (more than 50 points). This observation was confirmed by post-hoc analyses (from the second stimulus onwards, $\sigma$ 's ranged from 8 to 25 ). A further explanation may be the homogeneous and healthy group of subjects tested. Most subjects were young students with no current pain complaints. Lastly, in an experimental setting, differences in subjective pain ratings may not be visible. In a clinical setting, however, pain has more implications for a person and thus variability in pain experience may appear.

The second aim of this study was to demonstrate that subfacets of $\mathrm{N}$ exert different and independent influences on the pain-ERP. The results not only showed independent effects for $\mathrm{N}$-anx and N-dep, but they also were differentially associated indicating a negative association for $\mathrm{N}$-anx and a positive association for $\mathrm{N}$-dep with the pain-ERP amplitude. This directionally opposite association is remarkable considering that $\mathrm{N}$-anx and $\mathrm{N}$-dep correlate 0.7 within the NEO Personality Inventory (Costa \& McCrae, 1985). There was one latency range, however, where $\mathrm{N}$-anx was associated with an increase in amplitude. A post-hoc explanation might be that this represents pain-related trait anxiety whereas the latencies with amplitude reducing effects reflect non-pain-related trait anxiety. This is not in accordance 
with Gibson et al who found, as outlined above, an P400 augmenting role of anxiety. The reason for this discrepancy is unclear. Possibly methodological differences in stimulation method and especially the ERP quantification method may be brought to bear as an explanation. A dual role for anxiety in relation to pain has been suggested previously (Arntz et al, 1993). The influence of anxiety on pain is dependent on whether the anxiety is pain-related or not. Pain-related anxiety seems to augment pain ratings whereas anxiety unrelated to pain reduces pain ratings. Attention possibly plays a mediating role in this.

In this study, a relatively homogeneous group of subjects was tested. The participants were mainly university students aged 20 - 25 years. This sampling restriction may limit the generalizability of the results. Replication in other (patient) samples is thus required. Furthermore, despite the relatively robust effects, several other issues must be considered in interpreting the results. First, due to the complexity on two levels (the structure of a multitude of positive and negative peaks with large variability of a general ERP and the large amount of significant latency ranges in the results) the present findings should be interpreted with care. Therefore, replication of the present results is of great importance. Second, the ERP amplitudes are highly delicate and can be influenced by a large number of factors. Third, in contrast to most ERP studies, statistical analyses were not limited a priori to ERP components such as the N200 and the P300. Instead, an alternative analysis method was used (Lousberg, unpublished data). Although this gives an interesting view on how the variance around the ERP curve can be explained, over-interpretation of the results may ensue. Therefore, great care was taken in determining the pattern of associations.

In conclusion, different facets of the personality trait neuroticism ( $N$ ) may represent different moderating influences on the cortical processing of pain. If the present results can be replicated, it may be possible to gain valuable new insights into the complex relationship between the experience of pain, cortical processing of pain and individual differences therein at the experiential and psychophysiological level. 


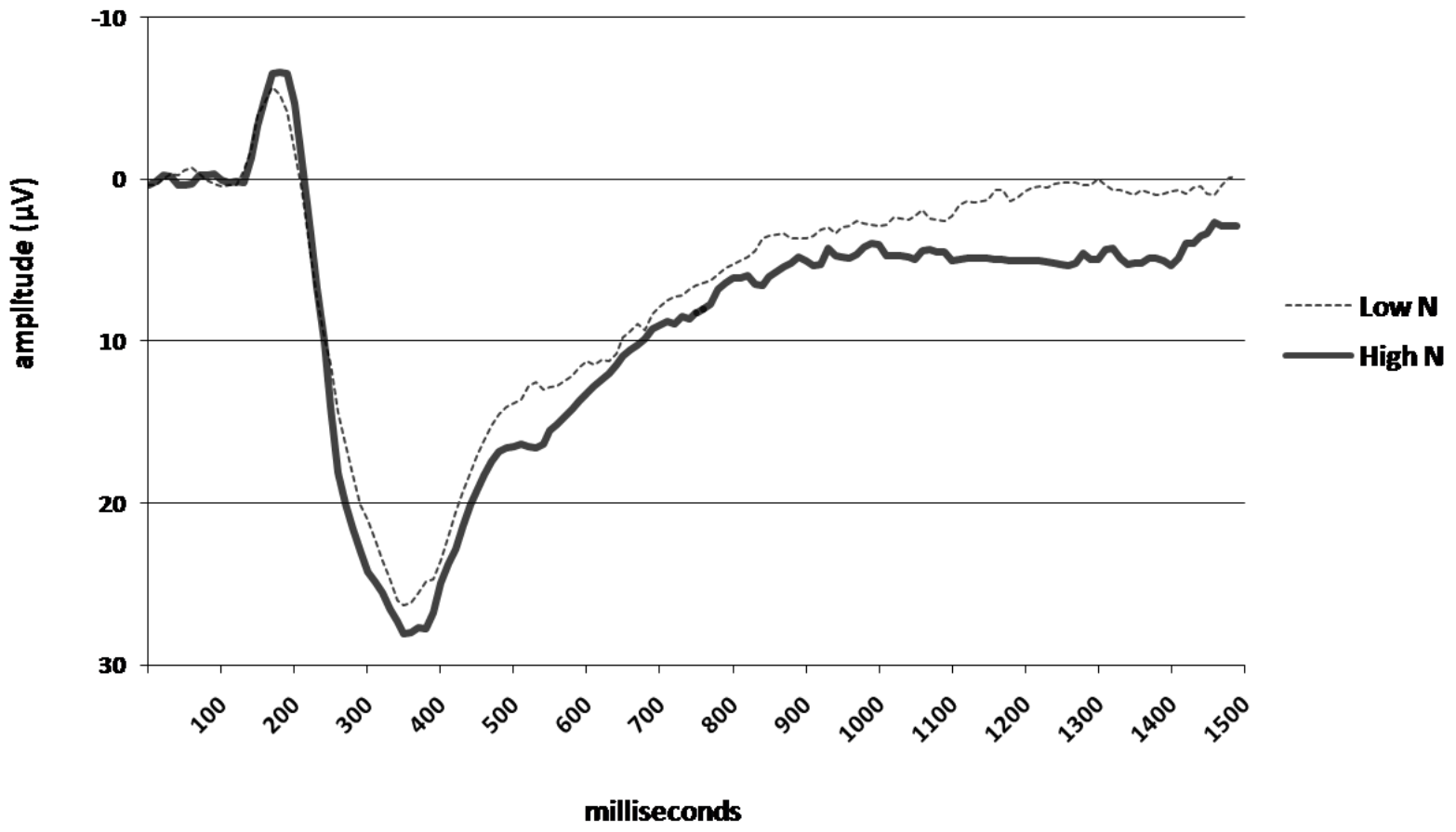

Figure 1a pain-ERP curves for low and high neuroticism sub groups $(C z)$

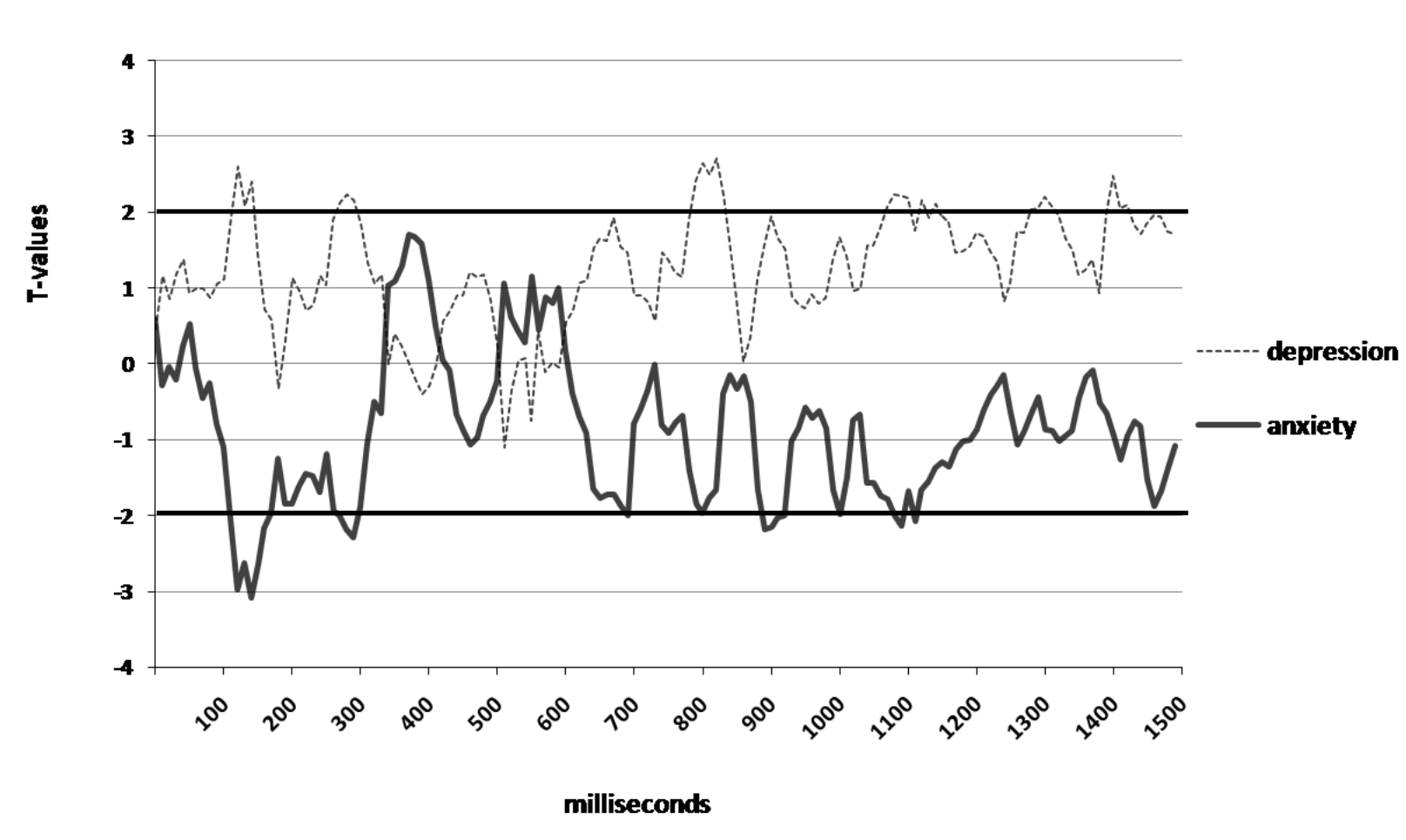

Figure 1b T-value curves for the moderating effects of anxiety and depression on the pain ERP amplitudes for each $10 \mathrm{~ms}(\mathrm{Cz})$ 


\section{References}

Arnzt A, de Jong PF (1993) Anxiety, attention and pain. Journal of Psychosomomatic Research, 37, 423-431.

Becker DE, Yingling CD, Fein G (1993) Identification of pain, intensity and P300 components in the pain evoked potential. Electroencephalography and Clinical Neurophysiology, 88, 290-301.

BenDebba M, Torgerson WS, Long DM (1997) Personality traits, pain duration and severity, functional impairment, and psychological distress in patients with persistent low back pain. Pain, 72, 115-125.

Bromm B, Meier W (1984) The intracutaneous stimulus: a new pain model for algesimetric studies. Methods \& Findings in Experimental and Clinical Pharmacology, 6, 405-410.

Costa PT, McCrae RR (1980) Somatic complaints in males as a function of age and neuroticism: A longitudinal analysis. Journal of Behavioural Medicine, 3, 245-257.

Costa PT, McCrae RR (1985) The NEO Personality inventory Manual. Odessa, Florida: Psychological Assessment Resources Inc.

Feeney SL (2004) The relationship between pain and negative affect in older adults. Journal of Anxiety Disorders, 18, 733-744.

Gibson SJ, LeVasseur SA, Helme RD (1991) Cerebral event-related responses induced by $\mathrm{CO}_{2}$ laser stimulation in subjects suffering from cervico-brachial syndrome. Pain, 47, 173-182.

32 Groth-Marnat G, Fletcher A (2000) Influence of Neurotcism, Catastrophizing, Pain Duration, and Receipt of Compensation on Short-Term Response to Nerve Block Treatment for Chronic Back Pain. Journal of Behavioural Medicine, 23, 339-35.

Hair JF, Anderson RE, Tatham RL, Black WC. Multivariate Data Analysis, fifth edition. New Jersey: Prentice Hall, 1998.

Hansenne M, Pitchot W, Pinto E, Reggers J, Papart P, Ansseau M (2000) P300 event-related brain potentials and personality in depression. European Psychiatry, 15, 370-377.

Harkins SW, Price DD, Braith J (1989) Effects of extraversion and neuroticism on experimental pain, clinical pain and illness behavior. Pain, 36, 209-218.

Hoekstra HA, Ormel J, de Fruyt F. NEO-PI-R - NEO FFI. Big flve persoonlijkheidsvragenlijsten. Handleiding. Lisse: Swets \& Zeitlinger, 1996.

Jasper HH (1958) The ten-twenty electrode system of the International Federation. Journal of Electroencephalography and Clinical Neurophysiology, 20, 371-375.

Johnson M (2003) The vulnerability status of neuroticism: over-reporting or genuine complaints? Personality and Individual Differences, 35, 877-887.

Keogh E, Hamid R, Hamid, S, Ellery D (2004) Investigating the effect of anxiety sensitivity, gender and negative interpretative bias on the perception of chest pain. Pain, 111, 209-217.

Lépine JP, Briley M (2004) The epidemiology of pain in depression. Human Psychopharmacology: Clinical and Experimental, 19, S3-S7. 
Lousberg R, Vossen HGM, Maessen C. Unraveling the Pain-ERP using an alternative approach: first results. Unpublished data.

McWilliams LA, Goodwin RD, Cox BJ (2004) Depression and anxiety associated with three pain conditions: results from a nationally representative sample. Pain, 111, 77-83.

Melzack R, Wall P. (1965) Pain mechanisms: A new theory. Science, 50, 971-979.

Miltner W, Johnson R, Braun C (1989) Somatosensory event-related potentials to painful and non-painful stimuli: effects of attention. Pain, 38, 303-312.

Munafo MR, Stevenson J (2001) Anxiety and surgical recovery. Journal of Psychosomatic Research, 51, 589-596.

Tang J, Gibson SJ (2005) A Psychophysical Evaluation of the Relationship Between Trait Anxiety, Pain Perception, and Induced State Anxiety. Journal of Pain, 6, 612-619.

Wade JB, Dougherty LM, Hart RP, Rafii A, et al. (1992) A canonical correlation analysis of the influence of neuroticism and extraversion on chronic pain, suffering, and pain behavior. Pain, 1, 67-73. 



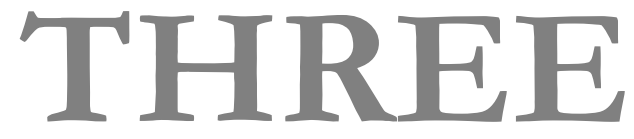

\title{
More Potential in Statistical Analyses of Event-Related Potentials:
}

\author{
A Mixed Regression Approach
}

\section{Reference}

Vossen HGM, Van Breukelen GJP, Hermens H, Van Os J, Lousberg R (2009) More Potential in Statistical Analyses of Event-Related Potentials: A Multilevel Approach. Submitted for publication 


\section{Abstract}

Despite many developments in the methods of Event-Related Potentials (ERP's), little attention has gone out to the statistical handling of ERP data. Trials are often averaged, and univariate or repeated measures ANOVA are used to test hypotheses. The aim of this study was to introduce mixed regression to ERP research and to demonstrate advantages associated with this method. Eighty-five healthy subjects received electrical pain stimuli with simultaneous EEG registration. Analyses first showed that results obtained with mixed regression analyses are highly comparable to those using repeated measures ANOVA. Second, important advantages of the mixed regression technique were demonstrated by allowing the inclusion of persons with missing data, single trial analysis, non-linear time effects, time*person effects (random slope effects) and a within-subject covariate. Among others, the results showed a strong trial (habituation) effect, which contraindicates the common procedure of averaging of trials. Furthermore, the regression coefficients for intensity and trial varied significantly between persons, indicating individual differences in the effect of intensity and trial on the ERP amplitude. In conclusion, using mixed regression analysis as a statistical technique in ERP research will advance the science of unraveling mechanisms underlying ERP data. 


\section{Introduction}

Event-Related Potentials (ERP's) are of value in many areas of research (Katadaet al, 2004; Porjesz et al, 2005; Hansenne, 2006). In pain research, for example, this time-locked derivative of EEG has frequently been used as a more objective measure of pain (Miltner et al, 1987; Granovsky et al, 2008). Over the last few decades, methodological developments have focused mainly on acquisition, pre-processing (Farwell et al, 1993; Spyzou et al, 2008) and component or signal identification of the ERP (Chapman et al, 1995; Samar et al, 1999; Jung et al, 2001). Relatively little attention has gone out, however, to progress in the statistical methods to test hypotheses in ERP research.

A common way of analyzing ERP's consists of (1) creating ERP's by averaging single trials of EEG (epochs) per individual, (2) subsequent averaging across individuals (per experimental condition), (3) determining the components and latency windows surrounding these components (where the experimental conditions differ most), (4) at the subject-level: calculation of the maximum amplitudes (within the latency windows) and (5) using these maximum amplitudes as a dependent variable in parametric statistical analyses such as ANOVA (Hoormann et al, 1998).

Although the common way of analyzing, as described above, is plausible and functional, there are at least two issues that need further consideration. First, each time-locked EEG record consists of a signal and a noise element. Because this signal element is considered to be constant over trials whereas the noise element is random, averaging trials will separate the signal form the noise. However, the assumption that the signal is constant over trials is not valid when processes such as habituation are expected (Woestenburg et al, 1983). Furthermore, recent studies indicate that background noise (oscillatory brain activity) cannot be "averaged out" due to asymmetric amplitude fluctuations, (Nikulin et al, 2007; Mazaheri \& Jensen, 2008). In addition, as stated above, by averaging trials, within-subject variance (trial-to-trial variance) is lost which may contain (clinically) important information on processes such as habituation. Often this problem is dealt with by averaging sequential blocks of trials (e.g. the first 10 trials, second 10 trials and so on). In this manner, a time-effect can be included as a withinsubject variable in repeated measures ANOVA. Nevertheless, a substantial part of the within-subject variance will be lost. Second, in order to make valid averages, a minimum of EOG-artefact free trials is needed. In most studies, a minimum of 5 to 10 trials is used. Since ANOVA deletes cases listwise, subject that do not meet this criterion are lost in the analyses. This can result in a substantial loss of analyzable cases. 
In this paper, an alternative way of statistical ERP analysis is proposed: mixed regression analysis (Snijders \& Bosker, 2000). The mixed regression technique (also mixed regression effects regression) is particularly appropriate for datasets with a hierarchical structure such as ERP data, as latency points are nested within a single trial and trials are nested within a single person. Although such data, as stated above, can also be analyzed with repeated measures ANOVA, mixed regression analysis has some important advantages: First, mixed regression analysis allows a large number of repeated measures per person without needing very large numbers of subjects. Aggregation of ERP trials is no longer necessary and single-trial data can be included in the analysis. There are other techniques that use single trial data, such as Wavelet analysis and Principal Component Analysis (Bostanov \& Kotchoubey, 2006). However, in these techniques single trial data is used for ERP signal detection and are subsequently averaged to compare experimental conditions. Second, missing data neither leads to listwise deletion of persons nor does it require imputation (Verbeke \& Molenberghs, 2000). This means that all valid (EOG-artifact free) trials can be included even if a person has just one valid trial. Third, mixed regression analysis is flexible in allowing time-dependent covariates and all kinds of correlation structures. Four, the mixed regression technique can incorporate random effects. These person-by-time effects make it possible to study individual differences over trials. Finally, mixed regression analyses is not restricted to the polynomial time contrast (which ANOVA is) but can also include, for example, exponential contrasts. This is an important advantage since repeated stimulation effects (such as in ERP paradigms) are often characterized by an exponential function (Timmermann et al, 2001).

This study aims at demonstrating that the mixed regression technique is a more flexible and feasible alternative to repeated measures ANOVA for ERP analysis. In order to achieve this goal, it will first be demonstrated that mixed regression and repeated measures ANOVA can produce comparable results. Next, the advantages of mixed regression will be demonstrated by (i) including all valid trials of all cases, regardless of the amount of EOG contaminated trials, (ii) by including exponential trial effects, (iii) by testing individual differences with random effects and (iv) by including a within-subject covariate: intensity of the previous trial. This alternative statistical technique is demonstrated using ERP data of healthy subjects receiving pain stimuli of which the intensity was under experimental control. 


\section{Materials and Method}

\section{Subjects}

Eighty-five pain-free subjects participated in the study. The age ranged from 18 to 65 years. Exclusion criteria were the use of analgesics and psychoactive drugs. Participation was rewarded with $€ 25,-$.

\section{Stimuli}

Stimuli used in this study were electrical pulses, of 10 milliseconds duration, that were administered intracutaneuously on the left middle finger. Five different intensities were presented based on the sensation threshold and the pain threshold. The sensation and pain threshold were determined by gradually increasing the intensity of the stimulus, starting at zero intensity. The first intensity that was consciously experienced was defined as the sensation threshold and the first intensity that was experienced as painful was defined as the pain threshold. This procedure was repeated three times in order to obtain a reliable measurement. One of the five intensities was the pain threshold and the other four intensities were defined relative to this pain threshold; $-50 \%,-25 \%,+25 \%$ and $+50 \%$ of the difference between the sensation threshold and the pain threshold (threshold range).

\section{Paradigm}

The stimuli were presented using a rating paradigm (Bromm, \& Meier, 1984). This paradigm consists of 150 stimuli. The five intensities, as mentioned above, were presented semi-randomly. Blocks of 15 stimuli were presented in which each stimulus intensity occurred three times. Inter Stimulus Interval (ISI) ranged between 9 and 11 seconds.

\section{EEG recording}

All EEG recordings were conducted in an electrically- and sound-shielded cubicle $\left(3 * 4 \mathrm{~m}^{2}\right) . \mathrm{Ag} / \mathrm{AgCl}$ electrodes were placed on $\mathrm{Fz}, \mathrm{Cz}, \mathrm{Pz}, \mathrm{C} 3, \mathrm{C} 4, \mathrm{~T} 3$ and T4 using the international 10-20 system (Jaspers 1958). Impedances were kept below $5 \mathrm{k} \Omega$. A reference electrode was placed on each ear lobe. To control for possible vertical eye movements, an electro-oculogram (EOG) electrode was placed 1 centimeter under the midline of the right eye. A ground electrode was placed at Fpz. All electrodes were fixed using 10-20 conductive paste. Neuroscan 4.3 software was used for EEG recording. 


\section{Procedure}

Before starting the experiment, subjects were informed about the purpose of the study. Subjects were told that they would undergo EEG-registration while they received various intensities of electric shocks some painless, some painful. After signing the informed consent form, EEG electrodes were placed and the shock electrode was attached to the top of the left middle finger as described by Bromm \& Meier, (1984). Next, the sensation and pain threshold were determined and after that, the rating paradigm was initiated.

\section{Data reduction}

EEG was recorded with $1000 \mathrm{~Hz}$ sampling rate, using Neuroscan 4.3 software. Trials were selected from the continuous EEG, from $200 \mathrm{~ms}$ prior to the stimulus until $1500 \mathrm{~ms}$ post-stimulus. Data was offline filtered (bandpass $0-50 \mathrm{~Hz}$ ) and baseline corrected based on $200 \mathrm{msec}$ pre-stimulus activity. Trials with EOG activity exceeding $+75 \mu \mathrm{V}$ and $-75 \mu \mathrm{V}$ were excluded from the analyses. For the ANOVA analyses and multilevel analyses of block averages, only subjects with 10 or more usable trials out of 30 per intensity were included in the statistical analyses. For the multilevel analyses of single trial data all EOG-artefact free trials from all subjects were included.

\section{Statistical analyses}

For the ANOVA analyses, maximum amplitudes were calculated for the N1 (latency range 20-55 msec), P1 (latency range 55-95 msec), N2 (latency range 95-145 msec) and P2 (latency range 145-300 msec) per intensity and cranial location per trial and then averaged across trials per block of 10 successive trials per person per intensity per cranial location per component. These components have been shown to be related to the processing of stimulus intensity (Kanda et al, 2002; Becker et al, 2000; Zaslansky et al, 1996; Bromm, 1984). To include a time-effect into the model, the data was divided into three blocks of 10 trials per intensity. Thus, the within-subject variables were intensity and block. Polynomial contrasts for block and intensity were incorporated. The ANOVA analyses were done separately for each peak and cranial location.

The mixed regression method was applied in two steps (see figure 1). In the first step, the same data were used as in the ANOVA analysis in order to show the similarities between both methods. In the second analyses, single trial data instead of block averages were analyzed, allowing (a) the inclusion of all trials without EOG activity and (b) the study of within-subject covariates such as stimulus intensity at 
the previous trial or specific functions of trial number to model hypothesized trend effects other than linear or quadratic due to e.g. habituation.

For the mixed regression analyses the same latency ranges were used as for the ANOVA method. The maximum amplitudes per trial per person were the dependent variables. Aside from intensity and trial, age, absolute stimulus intensity level of the sensation threshold and the pain threshold were included as covariates in this model. Gender was included as a between-subject variable. The covariates were centered (the sample mean was subtracted from each individual score) (Van Breukelen, \& Van Dijk, 2007). The rationale for these covariates and between-subject variables comes from studies that show a relation between these variables and pain or ERP (McDowell et al, 2003; Vallerand, \& Polomano, 2000) Thus, intensity, trial, age, gender and absolute stimulus intensity level of the sensation threshold and pain threshold were the independent variables (fixed factors). In this model, the intercept was allowed to vary randomly between subjects in order to accommodate interpersonal differences in average ERP. Also, the slopes of intensity and trial were allowed to vary randomly in order to accommodate interpersonal differences in effects of intensity and trial. The analyses were done separately for each component and cranial location. A description of the full mixed regression model is given in the appendix.

All statistical analyses were performed with SPSS 15.0. All two-tailed p-values $<=0.007$ (Bonferroni correction for the number of cranial locations (7)) were considered as statistically significant.

\section{Results}

\section{Step 1: repeated measures ANOVA vs mixed regression}

Of the 85 subjects, 29 were excluded due to excessive eye movements, resulting in 56 analyzable sets of participant data. The group consisted of 21 men (37.5\%) and 35 women (62.5\%). The mean age of the participants was 35 years $(S D=13.40)$. Figure $2 a$ shows the four components of interest in this study: $\mathrm{N} 1, \mathrm{P} 1, \mathrm{~N} 2$ and $\mathrm{P} 2$ at $\mathrm{Cz}$, averaged across all 56 subjects and all trials without EOG activity. Visual inspection of figure $2 \mathrm{a}$ indicates that the intensities have different amplitudes: as the intensity increases so does the peak amplitude. Figure $2 \mathrm{~b}$ shows the averages per block of 10 successive trials for $\mathrm{Cz}$ at the strongest stimulus intensity. This figure indicates that as the block increases, the peak amplitude decreases in a nonlinear fashion, suggesting habituation. The difference between the first and second block is larger than the difference between the second and third block. Figure 3 shows the averaged 
maximum peak amplitudes per intensity of the P2-component at $\mathrm{Cz}$. This figure displays a combination of the intensity and block effects of figures $2 a$ and $2 b$.

For the comparison of repeated measures ANOVA and the mixed regression technique, a simple model, following a 3 (block) by 5 (intensity) within-subject design, was chosen. For the sake of simplicity, no covariates were included into the analyses. In order to show that mixed regression analysis produces comparable results to repeated measures ANOVA, the mixed regression model was adapted in such a way that it most resembled the repeated measures ANOVA. This meant that the same cases were included (56 out of 85 cases), the same averaged blocks of trials and the same parameters were included in the model. Furthermore, since in repeated measures ANOVA polynomial contrasts are used, a linear and quadratic contrast for the within-subject variables intensity and block were included in the mixed regression model. The cubic and quadratic contrasts for intensity were not included in the mixed regression model since significant effects of these contrasts were not expected and these contrasts have no clinical (physiological) meaning. Finally, a Compound Symmetry Heterogeneous (CSH) covariance structure was chosen in the mixed regression analyses as a compromise between compound symmetry (which is close to the sphericity assumption of the univariate ANOVA method for repeated measures) and unstructured (which is equivalent to the assumption made by the multivariate ANOVA method). CSH means that the correlation between repeated measures is the same for each pair of repeated measures, but that the variance may differ between the repeated measures, which implies that the covariances may also differ between pairs ${ }^{1}$.

The $p$-values of the linear and quadratic contrasts for the within-subject variables (intensity and block) are depicted in table 1 . This table shows overall highly comparable results in the sense of statistically significant and marginally significant effects. No significant interactions between intensity and block were found and therefore these effects are not shown in table 1. Due to the centred coding of linear and quadratic contrasts, the interaction terms were orthogonal to the main effects.

In sum, the comparison of ANOVA repeated measures and mixed regression analyses shows that these two methods can produce comparable results.

1 Note that 3 blocks by 5 intensities gives 15 repeated measures and 105 pairs of measures, which implies that choosing an unstructured covariance matrix in mixed regression involves 120 unknown covariance parameters. For this reason, a fully unstructured covariance matrix is often feasible in mixed regression analysis only for a small number of repeated measures, e.g. up to 6 . 


\section{Step 2: The advantages of the mixed regression technique}

The advantages of the mixed regression technique are demonstrated in three parts. First, single trials are introduced in the analyses. Then random slopes are added to the model and finally a within-subject covariate is tested.

In mixed regression all valid trials from all participants can be included. This resulted in 85 analyzable cases of whom $33(38.8 \%)$ were men and $52(61.2 \%)$ women. T-tests and a chi-square test showed that the 29 extra participants did not differ from the 56 participants in the previous ANOVA / mixed regression analyses with respect to peak amplitude (mean of all valid trials) $(p>0.100)$, absolute pain threshold $(p=0.972)$, absolute sensation threshold $(p=0.940)$, age $(p=0.971)$ or gender $(p=0.793)$. Figure 4 displays the trial averages per intensity. It shows saw-toothed-like curves caused by the semirandom presentation of the five intensities (which causes the successive points on the curve for a given intensity to be based on different persons) as well as by intra-individual variation. Furthermore, an overall decrease over trials is visible.

\section{Random intercept models}

The model consisted of the peak amplitude (N1, P1, N2, P2 components) as dependent variable while

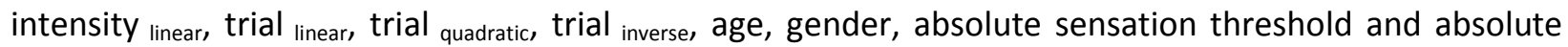

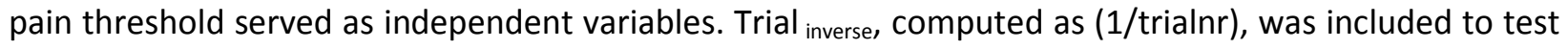
for nonlinear habituation effects, that is, for a decrease of peak amplitude which is strong at the start of a subject's session and gets smaller and smaller over the course of the session. Except for trial ${ }_{\text {quadratic }}$ and trial inverse, all predictors were centred (for details, see the model in the appendix). Given that no significant block*intensity interactions were found in the analyses of the first hypothesis, no interactions were included in these analyses. Since the number of repeated measures was now 150 (trials) instead of 15 as before, the most general covariance structure that could be fitted was ARMA1.1, which can handle both white noise (measurement error) and coloured noise (AR1, MA1). The intercept was allowed to vary randomly. The Goodness of fit of that covariance structure was significantly better than that of its competitors Compound Symmetry (CS) and AR1. The results of the analyses are displayed in table 2 .

The results demonstrate that the linear, quadratic and inverse effect of trial are statistically significant at multiple cranial locations and components. In order to interpret the size of the effects (B-values) it should be kept in mind that trial linear runs from -75 to +75 , trial quadratic runs from 0 to +5625 and trial inverse is coded from 1 to almost 0 . This means that the absolute $B$-values cannot be compared to each other. The results show that the linear effect of trials is the strongest over all locations in terms of 
p-value. Also the quadratic effect is significant at multiple locations, especially on the N2 component. In addition to the linear and quadratic effect, the inverse effect is significant at CzN1, C3P2 and T3N1.

Furthermore, although not displayed in the table, a significant effect of intensity (linear) was found on all components at all locations except for the N2 component at Pz and the N1 and P1 component at T3, T4 and C3. This was overall in accordance with the results of the previous analysis (except for CzP1 and C4P1). A significant effect for age was found on the P2-component at $\mathrm{Cz}$ and Pz. The other covariates did not show a significant effect on the amplitude of the ERP components.

In sum, these analyses demonstrated that the single trials of Event-Related Potential data differ from each other in that the amplitudes decrease over time. This decrement is a combination of a linear, a quadratic and an inverse trend.

\section{Random slope models}

Next, random slopes were added to the model. The random slopes that were included in this model were a random slope for intensity, a random slope for trial linear and a random slope for trial inverse. A random slope for trial quadric was initially also included but dropped in view of non-significance or convergence problems. The four random effects ( 3 slopes and 1 intercept) were initially allowed to correlate with each other (giving 10 unknown covariance parameters). In case of convergence problems that could not be solved by dropping the random slope for trial quadric, random effects were assumed to be uncorrelated, giving 4 instead of 10, or 3 instead of 6, covariance parameters. Furthermore, since no significant autocorrelation was found anymore after adding random effects, the repeated measures covariance structure was reduced to Scaled Identity (white noise). The resulting fixed effects ( $B^{\prime} s$ ) of the independent variables were highly similar to the results of the analyses without random effects. The resulting fixed effects and random effect variances are shown in table 3, showing significant intercept variance $\left(\sigma^{2}\right)$ at all locations and all components.

The slope variance of intensity is significant on some components of the central line (FzP1, CZN1, CzP1, CzN2) and C3P1, C3P2 C4N1, C4P1 and C4N2 implying inter-individual differences in the effect of intensity on ERP. The five intensities presented in the protocol were set relative to the individual difference in absolute sensation threshold and pain threshold (See the section Materials and Methods, subsection Stimuli). This means that the difference between the intensities was not equal for all subjects. However, the individual differences in the effect of intensity cannot be explained by these individual differences in absolute stimulus intensities, since a post-hoc analysis showed the slope 
variance to be still significant when using absolute intensity instead of relative intensity as predictor in the mixed regression model.

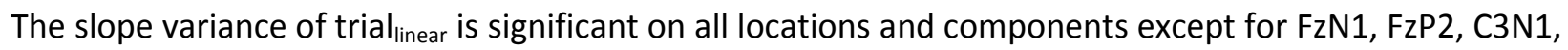
C4N1 and C4N2. The slope variance of trial ${ }_{\text {inverse }}$ is significant on all components of Fz and T3, on the N2component of T4 and the P2-components of C3, C4 and T4 and on none of the components of $\mathrm{Cz}$ and Pz. These analyses demonstrated that there are individual differences in the effects of intensity and trial on the ERP, which can be quantified in terms of between-subject variances in within-subject slopes for the regression of ERP on intensity and trial. This is a more refined way to study individual differences compared to repeated measures ANOVA of block averages.

\section{Within-subject covariate}

The final step was to include a within-subject covariate: intensity of the previous trial. This covariate was centred and then included as main effect and as part of an interaction term with intensity of the current trial. Random effects in this model were as in the previous model. The results showed that the main effect of the intensity of the previous trial was not significant at any of the cranial locations and ERP components. The interaction between the intensity of the previous trial and the intensity of the current trial was significant on $\mathrm{C} 3 \mathrm{~N} 2(p=0.003)$. Furthermore, on the other components of $\mathrm{C} 3$ this interaction was marginally significant ( $p$-values between 0.05 and 0.007 ). Finally, the results of the other independent variables (intensity, trial, age, gender, sensation and pain threshold) remained essentially the same after including the within-subject covariate

In sum, the large amount of results presented in this section demonstrate that (i) mixed regression analysis produces similar results compared to the more traditional repeated measures ANOVA, (ii) mixed regression analyses demonstrate that not only the linear effect of trial is significant, but also the quadratic and inverse effect and that these effects were mostly significant on the lateral cranial locations (see Table 2), (iii) the intercept and slopes of intensity and trial varied significantly between individuals and (iv) the within-subject covariate, stimulus of the previous intensity, was significant in interaction with the intensity of the present stimulus on C3N2. 


\section{Discussion}

The aim of this study was to demonstrate that the mixed regression technique is a flexible and appropriate method for analyzing Event-Related Potentials and that it has some important advantages over the traditional way of analysis (with repeated measures ANOVA).

Before demonstrating the advantages of the mixed regression technique, confirmation was provided that the mixed regression technique can provide the same results as repeated measures ANOVA. The $p$ values of the two analysis techniques were highly comparable. The small differences can be explained by the fact that repeated measures ANOVA uses an unstructured covariance structure and that for the mixed regression analyses a compound symmetry (heterogeneous) covariance structure was chosen ${ }^{2}$ which is somewhat less general than unstructured. Although assuming an unstructured covariance matrix is often preferable (since it is the most flexible covariance structure) and so a repeated measures ANOVA might seem better, there are some strong arguments in favour of mixed regression analysis Therefore, an attempt was made to show that the mixed regression technique has some important advantages over repeated measures AVOVA. A first advantage of the mixed regression technique is that single trial data can be used in the analyses. This not only allows inclusion of subjects with missing data, as mentioned earlier (which in turn increases the power and reduces the risk of selection bias), but also allows the study of time (trial) effects with any kind of function of trial number, including an inverse (1/trial) function. The results show that in addition to a strong linear trial effect and a fairly strong quadratic trial effect, the inverse trial effect was also significant at different components and locations. Furthermore, there were several marginally significant effects of the inverse trial effect ( $p$-values between 0.05 and 0.007 ). These results imply that the peak amplitudes of the ERP decrease over time (habituation) but that the way in which they decrease cannot merely be expressed in polynomial (linear and quadratic) contrasts. This is in accordance with the consensus that pain habituates in a non-linear fashion (Milne et al, 1991).

Another advantage is that in mixed regression analysis it is possible to study individual differences in the within-subject variance. In practice, this means that individual differences in the effect of intensity and trial on the cortical processing of stimuli can be investigated. These subject*time and subject*intensity interactions are represented as random slopes. In the analyses, a random intercept, random slope for intensity and two random slopes for trial (linear and inverse) were included. The results showed strong

\footnotetext{
2 Mixed regression analysis can only handle an unstructured covariance structure with a relatively small amount of repeated measures whereas ANOVA can handle many repeated measures as long as they are the result of a factorial design in which each within-subject factor has a small number of levels.
} 
intercept variance, representing differences in mean amplitude between the subjects. The significant slope variance for intensity represents individual differences in the effect of intensity. Furthermore, significant slope variance for trial linear and trial inverse was found, implying individual differences in the habituation to repeated measures. This is an interesting finding, because these individual differences in habituation might in part explain individual differences in the response to pain in general which in turn might explain the development of chronic pain complaints (Valeriani et al, 2003). These results can be used to form and test new hypotheses to explain these individual differences.

Finally, because single trial data was used in the analyses, covariates that vary over trials (within-subject covariate) can be investigated. In this study, the intensity of the previous trial was included in the model because of the semi-random presentation of the intensities in the paradigm. Our hypothesis was that the intensity of the previous stimulus influences the cortical reaction of the present stimulus. Moreover, it was expected that an interaction between the intensity of the current stimulus and the intensity of the previous stimulus (difference in intensity) would influence the cortical response to the current stimulus. The results did not confirm these hypotheses. No main effect of the intensity of the previous stimulus was found. However, a significant interaction effect was found on the N2-component of C3, meaning that the effect of the intensity of the current stimulus on the ERP amplitude is dependent of the intensity of the previous stimulus. Also marginally significant interaction effects ( $p$-values between 0.05 and 0.007 ) were found on the other components of C3. This might imply that this area is involved in the comparison of past and present intensities, but further research will have to confirm this.

Naturally, fine-tuning of this technique is necessary. For instance, relatively simple techniques for filtering and detection of components were used. The mixed regression analysis technique can also be used in combination with other techniques of component identification such as Principal Component Analysis or Wavelet analysis. Furthermore, in this study, the inverse function was calculated by dividing 1 by trial number. Of course there are other ways to calculate an inverse function which might even better fit the data.

In conclusion, this article has demonstrated on a theoretical as well as a practical level that the mixed regression technique offers important advantages for ERP research. The model given in this study can easily be adapted to different ERP paradigms (e.g. Oddball paradigms) and research questions by including different independent variables and different random effects. Using mixed regression analysis as a statistical technique in ERP research will advance the unravelling of mechanisms underlying the ERP. 


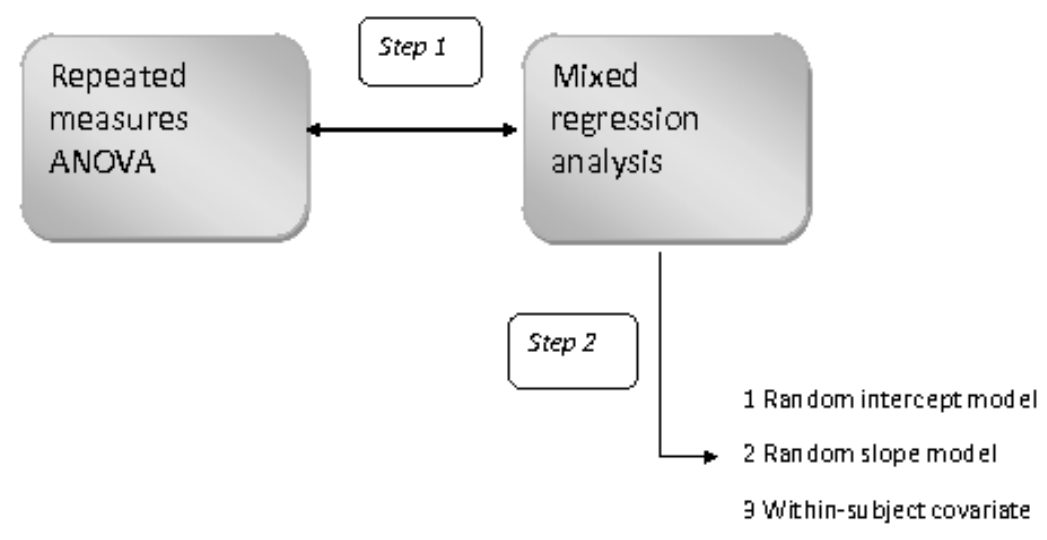

Figure 1 Graphic illustration of the applied models. Step 1 represents the comparison of the repeated measures ANOVA with the mixed regression analysis, with averaged data across trials. Step 2 represents a demonstration of the advantages of mixed regression analysis, using single trial data.

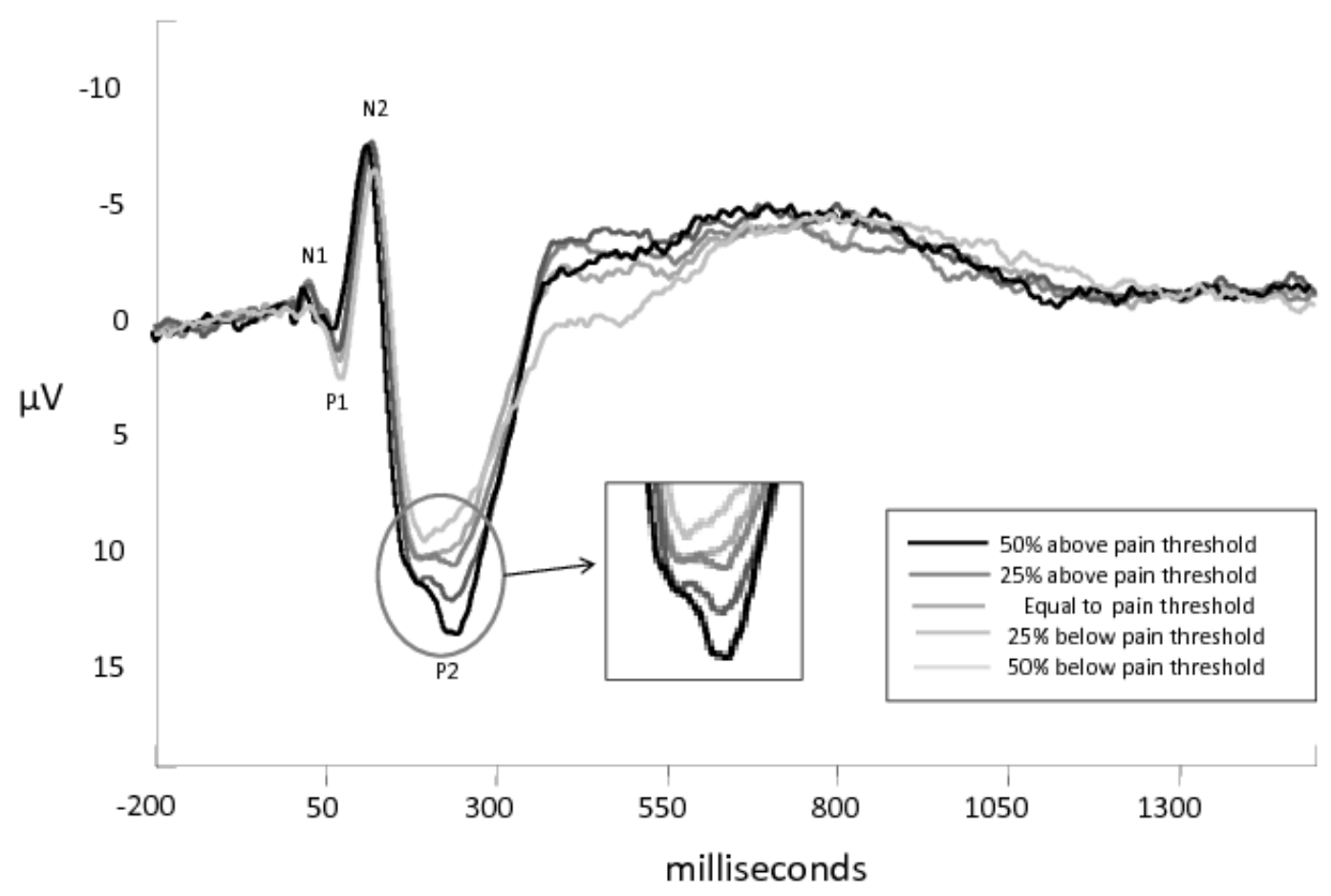

Figure 2a Grand Average per stimulus intensity on Cz. 


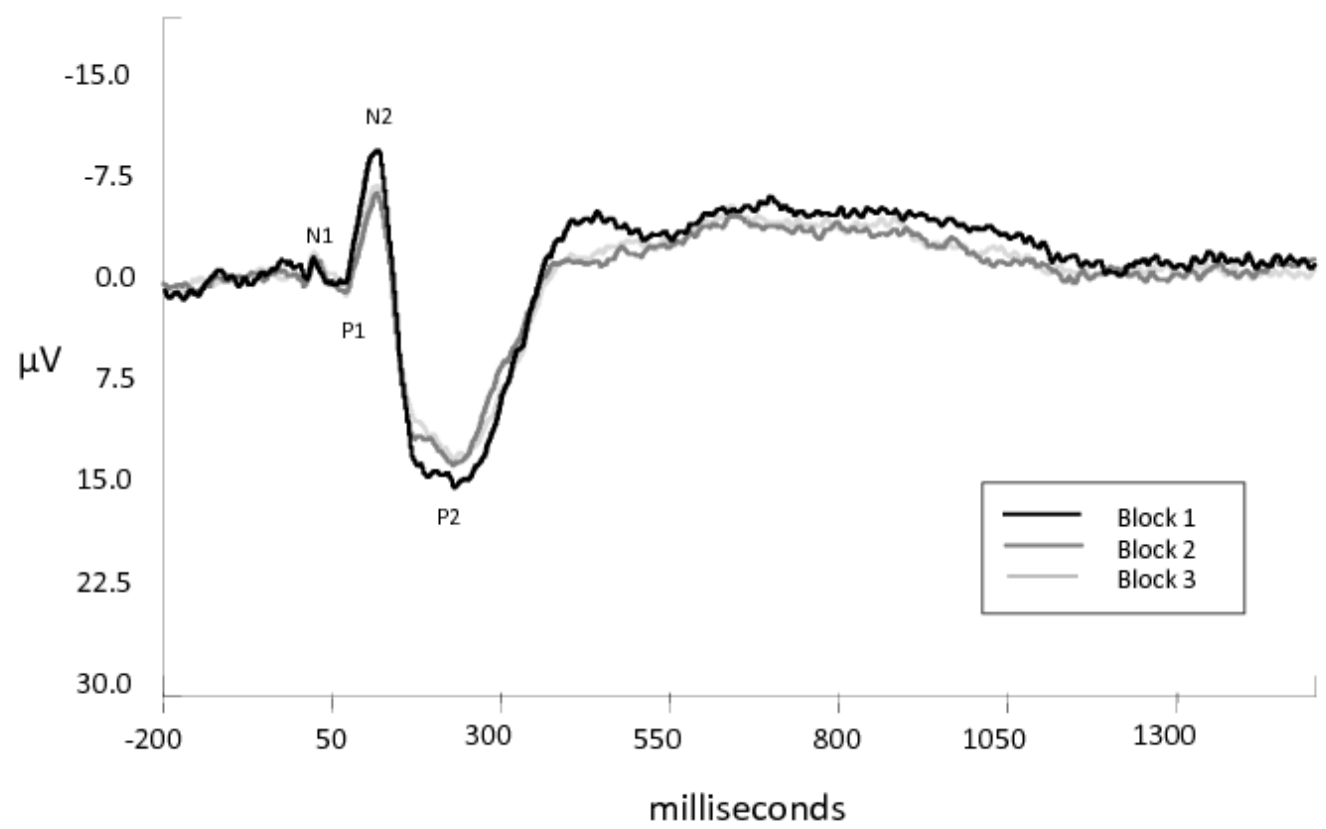

Figure $2 \mathrm{~b}$ Grand average per block at $\mathrm{Cz}$.

$\begin{array}{ll}\square & \square_{25 \%} 5 \% \text { below pain threshold } \\ \square_{25 \%} \text { above pain threshold } & \square_{50 \% \text { above pain threshold }}\end{array}$

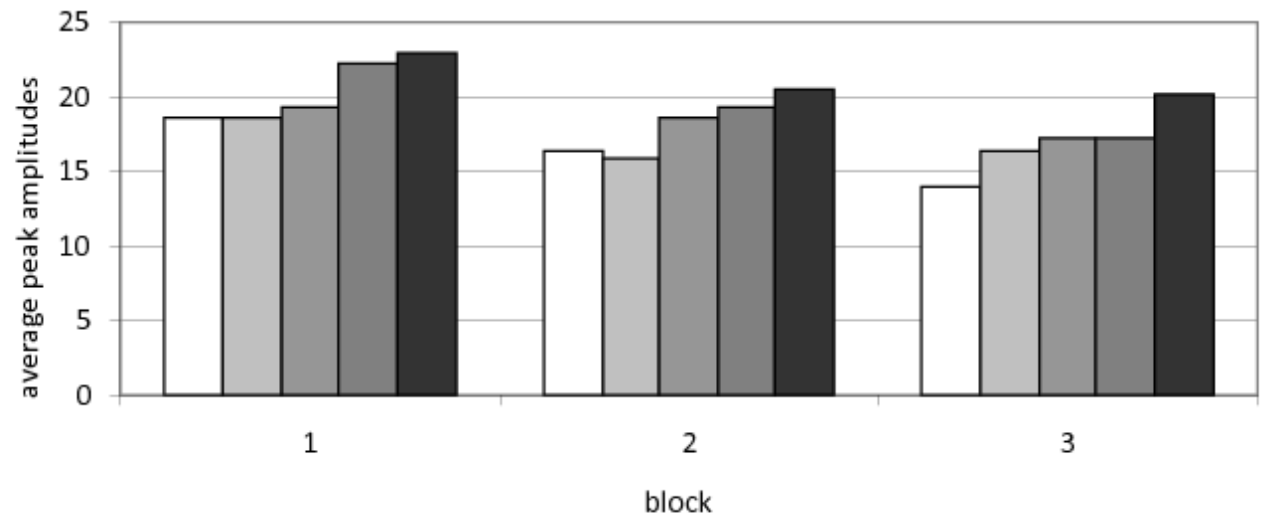

Figure 3 Block averages per intensity on CZP2. 

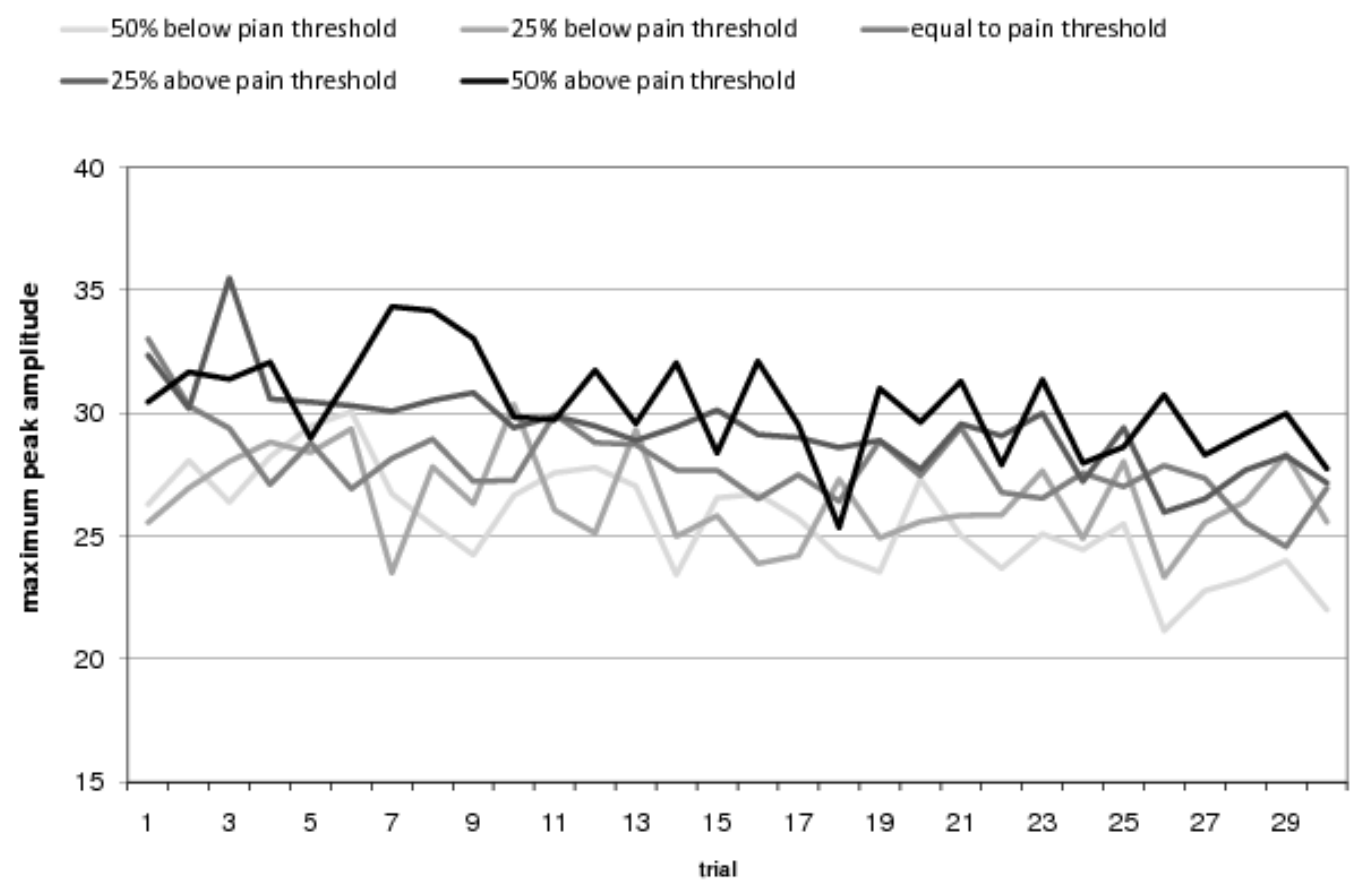

Figure 4 P2 peak amplitudes per intensity over trials 
Table 1 Comparison of $p$-values between repeated measures ANOVA and multilevel analysis. The linear and quadratic effects of intensity and block are depicted. Significant effects $(p<0.007)$ are displayed in bold and marginally $(p<0.05)$ significant effects are displayed in italics.

\begin{tabular}{|c|c|c|c|c|c|c|}
\hline \multicolumn{2}{|c|}{ location } & \multirow{3}{*}{$\begin{array}{l}\text { contrast } \\
\text { Linear } \\
\text { quadratic }\end{array}$} & \multirow{2}{*}{$\begin{array}{l}\text { ANOVA (intensity) } \\
p<0.001\end{array}$} & \multirow{2}{*}{$\begin{array}{l}\begin{array}{l}\text { Multilevel } \\
\text { (intensity) }\end{array} \\
p<0.001\end{array}$} & \multirow{2}{*}{$\begin{array}{l}\text { ANOVA (Block) } \\
p=0.561\end{array}$} & \multirow{2}{*}{$\begin{array}{l}\text { Multilevel (Block) } \\
p=0.517\end{array}$} \\
\hline $\mathrm{Fz}$ & N1 & & & & & \\
\hline & & & $p=0.052$ & $p=0.044$ & $p=0.617$ & $p=0.596$ \\
\hline & P1 & Linear & $p=0.145$ & $p=0.043$ & $p=0.031$ & $p=0.008$ \\
\hline & & quadratic & $\mathrm{p}=0.812$ & $p=0.922$ & $p=0.247$ & $p=0.233$ \\
\hline & N2 & Linear & $\mathrm{p}<0.001$ & $\mathrm{p}<0.001$ & $p<0.001$ & $p<0.001$ \\
\hline & & quadratic & $p=0.006$ & $p=0.030$ & $p=0.418$ & $p=0.150$ \\
\hline & $\mathrm{P} 2$ & Linear & $\mathrm{p}<0.001$ & $\mathrm{p}<0.001$ & $\mathrm{p}<0.001$ & $\mathrm{p}<0.001$ \\
\hline & & quadratic & $p=0.194$ & $p=0.107$ & $p=0.184$ & $p=0.300$ \\
\hline \multirow[t]{8}{*}{$\mathrm{Cz}$} & N1 & Linear & $\mathrm{p}=0.061$ & $p=0.051$ & $p=0.703$ & $p=0.736$ \\
\hline & & quadratic & $p=0.085$ & $p=0.075$ & $p=0.723$ & $p=0.678$ \\
\hline & P1 & Linear & $\mathrm{p}=0.313$ & $p=0.214$ & $p=0.377$ & $p=0.338$ \\
\hline & & quadratic & $p=0.361$ & $p=0.640$ & $p=0.656$ & $p=0.494$ \\
\hline & N2 & Linear & $p=0.006$ & $p=0.001$ & $p<0.001$ & $\mathrm{p}<0.001$ \\
\hline & & quadratic & $\mathrm{p}<0.001$ & $p=0.030$ & $p=0.030$ & $p=0.140$ \\
\hline & P2 & Linear & $\mathrm{p}<0.001$ & $\mathrm{p}<0.001$ & $p<0.001$ & $\mathrm{p}<0.001$ \\
\hline & & quadratic & $p=0.194$ & $p=0.264$ & $p=0.080$ & $p=0.090$ \\
\hline \multirow[t]{8}{*}{$\mathrm{Pz}$} & N1 & Linear & $\mathrm{p}=0.021$ & $p=0.023$ & $p=0.449$ & $p=0.325$ \\
\hline & & quadratic & $p=0.459$ & $p=0.575$ & $p=0.861$ & $p=0.812$ \\
\hline & P1 & Linear & $\mathrm{p}=0.014$ & $p=0.003$ & $p=0.879$ & $p=0.817$ \\
\hline & & quadratic & $p=0.186$ & $p=0.249$ & $p=0.838$ & $p=0.901$ \\
\hline & N2 & Linear & $p=0.692$ & $p=0.721$ & $\mathrm{p}<0.001$ & $\mathrm{p}<0.001$ \\
\hline & & quadratic & $p=0.054$ & $p=0.028$ & $p=0.486$ & $p=0.564$ \\
\hline & P2 & Linear & $\mathrm{p}<0.001$ & $p<0.001$ & $p<0.001$ & $p<0.001$ \\
\hline & & quadratic & $p=0.150$ & $p=0.135$ & $p=0.855$ & $p=0.916$ \\
\hline
\end{tabular}


Table 2 The estimates (B), standard errors (SE) and p-values for the linear, quadratic and inverse trial effect on $F z, C z, P z, C 4, C 3$, T3 and T4. Significant $(p<0.007)$ effects are displayed in bold and marginally $(p<0.05)$ significant effects are displayed in italics.

\begin{tabular}{|c|c|c|c|c|}
\hline \multicolumn{2}{|c|}{ Location } & \multirow{2}{*}{$\begin{array}{l}\text { Linear } \\
B=-0.002, S E=0.003, p=0.560\end{array}$} & \multirow{2}{*}{$\begin{array}{l}\text { Quadric } \\
\mathrm{B}=-6 \mathrm{E}-005, \mathrm{SE}=6.8 \mathrm{E}-005, \mathrm{p}=0.374\end{array}$} & \multirow{2}{*}{$\begin{array}{l}\text { Inverse } \\
\mathrm{B}=-1.80, \mathrm{SE}=1.23, \mathrm{p}=0.144\end{array}$} \\
\hline $\mathrm{Fz}$ & N1 & & & \\
\hline & P1 & $B=-0.006, S E=0.004, p=0.153$ & $B=-2 E-005, S E=8.2 E-005, p=0.822$ & $B=1.69, S E=1.44, p=0.240$ \\
\hline & N2 & $B=0.021, S E=0.004, p<0.001$ & $B=-0.0002, S E=8.7 E-005, p=0.009$ & $B=3.82, S E=1.58, p=0.016$ \\
\hline & $\mathrm{P} 2$ & $B=-0.012, S E=0.004, p<0.001$ & $\mathrm{~B}=9.6 \mathrm{E}-006, \mathrm{SE}=8.8 \mathrm{E}-005, \mathrm{p}=0.913$ & $B=4.08, S E=1.62, p=0.012$ \\
\hline \multirow[t]{4}{*}{$\mathrm{Cz}$} & N1 & $B=-0.004, S E=0.003, p=0.196$ & $B=5.6 E-005, S E=6.6 E-005, p=0.394$ & $B=-3.49, S E=1.19, p=0.003$ \\
\hline & P1 & $B=0.007, S E=0.035, p=0.038$ & $B=-0.00013, S E=7.5 E-005, p=0.082$ & $B=-1.86, S E=1.33, p=0.126$ \\
\hline & N2 & $B=0.040, S E=0.004, p<0.001$ & $\mathrm{~B}=-0.0003, \mathrm{SE}=8.8 \mathrm{E}-005, \mathrm{p}<0.001$ & $B=-2.51, S E=1.56, p=0.108$ \\
\hline & $\mathrm{P} 2$ & $B=-0.025, S E=0.005, p<0.001$ & $B=2.0 E-005, S E=9.9 E-005, p=0.837$ & $B=2.44, S E=1.65, p=0.140$ \\
\hline \multirow[t]{4}{*}{$\mathrm{Pz}$} & N1 & $B=-0.002, S E=0.003, p=0.553$ & $\mathrm{~B}=2.6 \mathrm{E}-005, \mathrm{SE}=8.6 \mathrm{E}-005, \mathrm{p}=0.697$ & $B=-1.14, S E=1.20, p=0.344$ \\
\hline & P1 & $B=0.006, S E=0.005, p=0.288$ & $B=-0.0002, S E=9.7 E-005, p=0.056$ & $B=-0.36, S E=1.31, p=0.782$ \\
\hline & N2 & $B=0.016, S E=0.004, p<0.001$ & $B=-0.0001, S E=8.1 E-005, p=0.142$ & $B=-1.07, S E=1.45, p=0.464$ \\
\hline & $\mathrm{P} 2$ & $B=-0.013, S E=0.005, p=0.008$ & $B=-5.0 E-005, S E=9.4 E-005, p=0.619$ & $B=2.68, S E=1.60, p=0.094$ \\
\hline \multirow[t]{4}{*}{ C3 } & N1 & $B=-0.002, S E=0.003, p=0.419$ & $B=-9.0 E-005, S E=5.8 E-005, p=0.125$ & $B=-2.07, S E=1.03, p=0.045$ \\
\hline & P1 & $B=0.001, S E=0.004, p=0.710$ & $B=-0.0002, S E=7.4 E-005, p=0.042$ & $B=0.77, S E=1.22, p=0.527$ \\
\hline & N2 & $B=0.027, S E=0.004, p<0.001$ & $B=-0.0003, S E=7.7 E-005, p=0.001$ & $B=-1.11, S E=1.33, p=0.407$ \\
\hline & $\mathrm{P} 2$ & $B=-0.017, S E=0.005, p<0.001$ & $B=-3.0 E-005, S E=8.4 E-005, p=0.761$ & $\mathrm{~B}=4.89, \mathrm{SE}=1.41, \mathrm{p}=0.001$ \\
\hline \multirow[t]{4}{*}{ C4 } & N1 & $B=-0.011, S E=0.003, p=0.001$ & $B=-0.0001, S E=6.4 E-005, p=0.001$ & $B=-1.20, S E=1.11, p=0.282$ \\
\hline & P1 & $B=0.021, S E=0.005, p<0.001$ & $B=-0.0001, S E=8.7 E-005, p=0.137$ & $B=-1.81, S E=1.30, p=0.137$ \\
\hline & N2 & $B=0.030, S E=0.004, p<0.001$ & $B=-0.0002, S E=7.8 E-005, p=0.004$ & $B=-1.96, S E=1.38, p=0.153$ \\
\hline & $\mathrm{P} 2$ & $B=-0.018, S E=0.006, p=0.001$ & $B=8.1 E-005, S E=9.9 E-005, p=0.417$ & $B=3.64, S E=1.51, p=0.016$ \\
\hline \multirow[t]{4}{*}{ T3 } & N1 & $B=0.013, S E=0.004, p=0.003$ & $B=-0.0003, S E=7.3 E-005, p<0.001$ & $B=-2.83 \mathrm{SE}=1.00, p=0.005$ \\
\hline & P1 & $B=-0.013, S E=0.004, p=0.002$ & $B=-0.0001, S E=7.1 E-005, p=0.045$ & $B=-0.87, S E=1.02, p=0.394$ \\
\hline & N2 & $B=0.031, S E=0.006, p<0.001$ & $B=-0.0003, S E=9.4 E-005, p=0.001$ & $B=-3.35, S E=1.26, p=0.008$ \\
\hline & $\mathrm{P} 2$ & $B=-0.029, S E=0.005, p<0.001$ & $\mathrm{~B}=0.0004, \mathrm{SE}=8.2 \mathrm{E}-005, \mathrm{p}<0.001$ & $\mathrm{~B}=1.22, \mathrm{SE}=1.23, \mathrm{p}=0.321$ \\
\hline \multirow[t]{4}{*}{ T4 } & N1 & $B=0.016, S E=0.004, p<0.001$ & $B=-0.0002, S E=7.6 E-006, p=0.009$ & $B=0.26, S E=1.55, p=0.823$ \\
\hline & P1 & $B=0.003, S E=0.004, p=0.461$ & $B=-0.0001, S E=7.3 E-005, p=0.076$ & $B=-0.46, S E=1.18, p=0.706$ \\
\hline & N2 & $B=0.028, S E=0.006, p<0.001$ & $B=-0.0002, S E=0.00011, p=0.053$ & $B=-3.50, S E=1.45, p=0.016$ \\
\hline & $\mathrm{P} 2$ & $B=-0.023, S E=0.005, p<0.001$ & $B=-0.0003, S E=9.6 E-005, p=0.004$ & $B=3.14, S E=1.48, p=0.034$ \\
\hline
\end{tabular}




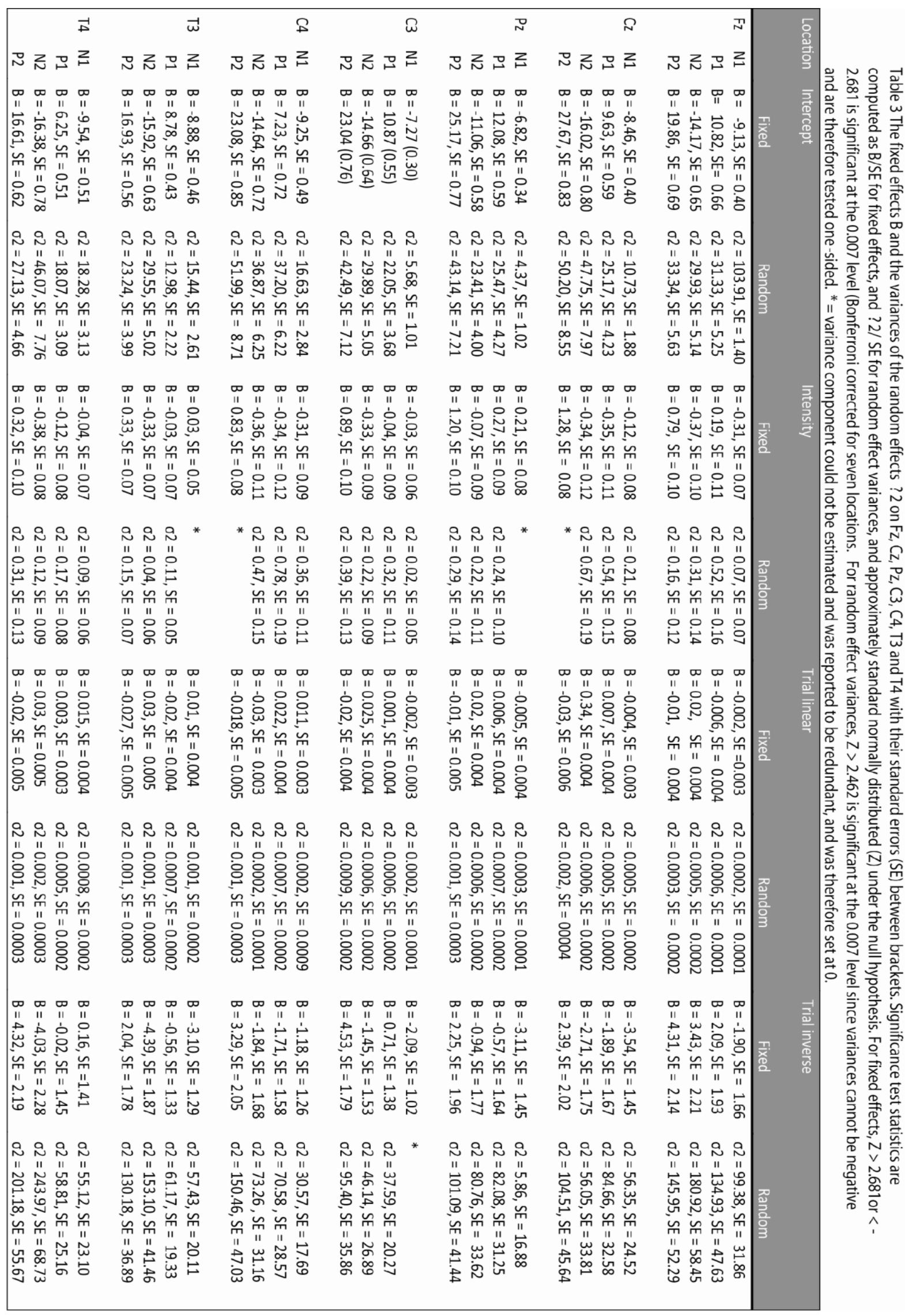




\section{Appendix: The full multilevel model}

$Y_{\mathrm{ti}}=\beta_{0}+\beta_{1}$ intensity linear $+\beta_{2}$ trial $_{\text {linear }}+\beta_{3}$ trial $_{\text {quadratic }}+\beta_{4}$ trial $_{\text {inverse }}+\beta_{5}$ age $+\beta_{6}$ gender $+\beta_{7}$ absolute sensation threshold $+\beta_{8}$

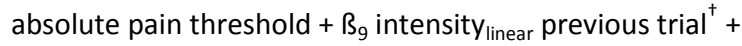

$\aleph_{10}$ intensity $_{\text {linear }} *$ intensity linear $_{\text {previous trial }}+e_{t i}+u_{0 i}+u_{1}$ intensity $y_{\text {linear }}+u_{2}$ trial $l_{\text {linear }}+u_{4}$ trial $l_{\text {inverse }}$.

${ }^{\dagger}$ The intensity of the previous trial was not included until the last step of the analyses.

Where:

$\mathrm{t}=$ time point (1 to 150$)$,

$\mathrm{i}=$ subject

Intensity $=-2=-50 \%,-1=-25 \%, 0=0 \%, 1=25 \%$ and $2=50 \%$,

Trial $=150$ trial numbers, centred from -74.5 to +74.5 ,

Age $=$ centred continuous variable in years,

Gender = dichotomous variable, $-1=$ man, 1= woman,

Absolute sensation threshold $=$ centred continuous variable in $\mathrm{mA}$,

Absolute pain threshold $=$ centred continuous variable in $\mathrm{mA}$,

Intensity previous trial $=-2=-50 \%,-1=-25 \%, 0=0 \%, 1=25 \%$ and $2=50 \%$,

$e_{t i}=$ error variance for subject $i$ at time point $t$. which is assumed to be the sum of an auto correlated component (AR1) plus

54 measurement error (white noise).

$\mathrm{u}_{0 \mathrm{i}}=$ personal deviation from the average intercept $\beta_{0}$

$\mathrm{u}_{1 \mathrm{i}}=$ personal deviation from the average slope $\aleph_{1}$ of intensity $y_{\text {linear }}$

$\mathrm{u}_{2 \mathrm{i}}=$ personal deviation from the average slope $\aleph_{2}$ of trial $\left.\right|_{\text {linear }}$

$\mathrm{U}_{4 \mathrm{i}}=$ personal deviation from the average slope $\aleph_{2}$ of trial linverse $_{\text {. }}$

The four random person effects are allowed to correlate with each other.

This model must be interpreted as follows:

$\beta_{0}=$ the outcome mean (Amplitude) for the intensity equal to the pain threshold (intensity $=0$ ) at trial number 75 for a subject with a mean age, mean absolute sensation threshold and mean absolute pain threshold.

$\beta_{1}=$ the mean difference between the adjacent intensities

$\beta_{2}=$ the mean change over trials according to the linear part of the trend

$\beta_{3}=$ the quadratic trial effect

$\aleph_{4}=$ the inverse trial effect

$\beta_{5}=$ the mean change in amplitude per year

$\beta_{6}=$ half the mean difference between men and women

$\beta_{7}=$ the relation between the absolute sensation threshold and the amplitude of the component

$\beta_{8}=$ the relation between the absolute pain threshold and the amplitude of the component

$B_{9}=$ the relation between the intensity of the previous trial and the amplitude of the present trial

$\beta_{10}=$ the effect of the intensity of the previous trial on the difference in amplitude between adjacent intensities (e.g. intensity 2 versus 1,) 


\section{References}

Becker DE, Haley DW, Urena VM, Yingling CD (2000) Pain measurement with evoked potentials: combination of subjective ratings, randomized intensities, and long interstimulus intervals procedures a P300-like confound. Pain, 84, 37-47.

Bostanov V, Kotchoubey B (2006) The t-CWT: A new ERP detection and quantification method based on the continuous wavelet transform and Student's t-statistics. Clinical Neurophysiology, 177, 2627-2644.

Bromm B (1984) Pain-related components in the cerebral potential: experimental and multivariate statistical approaches. In Pain measurement in man: Neurophysiological correlates of pain (Ed. Bromm B), pp. $257-290$. Elsevier: Amsterdam.

Bromm B, Meier W (1984) The intracutaneous stimulus: a new pain model for algesimetric studies. Methods and Findings in Experimental and Clinical Pharmacology, 6, 405-410.

Carrillo-de-la-Peňa MT (1992) ERP augmenting/reducing and sensation seeking: a critical review. International Journal of Psychophysiology, 12, 211-220.

Chapman RM, McCrary JW (1995) EP Component Identification and Measurement by Principal Component Analysis. Brain Cognition, 27, 288-310.

Farwell LA, Martinerie JM Bashore TR, Rapp PE, Goddard PH (1993) Optimal digital filters for long-latency components of event-related potentials. Psychophysiology, 30, 306-315.

Goldstein H (1999) Multilevel Statistical Analyses. London: Institution of Education, Multilevel Models Project. http://www.arnoldpublishers.com/support/goldstein.htm.

Granovsky Y, Granot M, Nir RR, Yarnitsky D (2008) Objective correlate of subjective pain perception by contact heat-evoked potentials. Journal of Pain, 9, 53-63.

Hansenne M (2006) Event-related Potentials in psychopathology: Clinical and Cognitive Perspectives. Psychologica-Belgica, 46, 5-36.

Hoormann J, Falkenstein M, Schwarzenau P, Hohnsbein J (1998) Methods for the quantification and statistical testing of ERP differences across conditions. Behavioural Research Methods Instruments \& Computers, 30, 103109.

Jasper $\mathrm{HH}$ (1958) The ten-twenty electrode system of the International Federation. Journal of Electroencephalography and Clinical Neurophysiology, 20, 371-375.

Jung TP, Makeig S, Westerfield JT, Courchesne E, Seijnowski T (2001) Analysis and Vizualization of Single-Trial Event-Related Potnetials. Human Brain Mapping, 14, 166-185.

Kanda M, Matsuhashi M, Sawamoto N, Oga T, Mima T, Nagamine T, et al. (2002) Cortical potentials related to assessment of pain intensity with visual analogue scale (VAS). Clinical Neurophysiology, 113, 1013-1024.

Katada E, Sato K., Ojika K, Ueda R (2004) Cognitive Event-related Potentials: Useful Clinical Information on Alzheimer's Disease. Current Alzheimer Research, 1, 63-69.

Mazaheri A, Jensen O (2008). Asymmetric Amplitude Modulations of Brain Oscillations Generate Slow Evoked Responses. The Journal of Neuroscience, 28, 7781-7787. 
McDowell K, Kerick SE, Santa Maria DL, Hatfield BD (2003) Aging, physical activity, and cognitive processing: an examination of P300. Neurobiological Aging, 24, 579-606.

Milne RJ, Kay NE, Irwin RJ (1991) Habituation to repeated painful and non-painful cutaneous stimuli: A quantitative psychophysiological study. Experimental Brain Research, 87, 438-444.

Miltner W, Larbig W, Braun C (1987) Habituation of subjective ratings and Event-related Potentials to painful intracutaneous electrical stimulation of the skin. Journal of Psychophysiology, 1, 221-228.

Nikulin VV, Linkenkaer-Hansen K, Nolte G, Lemm S, Müller KR, Ilmoniemi RJ, et al. (2007) A novel mechanism for evoked responses in the human brain. European Journal of Neuroscience, 25, 3146-3154.

Porjesz B, Rangaswamy M, Kamarajan C, Jones KA, Padmanabhapillai A, Begleiter H (2005) The utility of neurophysiological markers in the study of alcoholism. Clinical Neurophysiology, 116, 993-1018.

Samar VJ, Bopardikar A, Rao R, Swartz K (1995) Wavelet analysis of neuroelectroc waveforms: a conceptual tutorial. Brain and Language, 66, 7-60.

Snijders T, Bosker R (2000). Multilevel Analysis. SAGE Publications Ltd. London.

Schwerdtfeger A, Baltissen R (2002) Augmenting-reducing paradox lost ? A test of Davis et al.'s (1983) hypothesis. Personality and Individual Differences, 32, 257-271.

Spyzou L, Sanei S (2008) Source Localization of Event-Related Potentials incorporating spatial notch filters. IEEE Trandsactions on Biomedical Engineering, 55, 2232-2239.

Van Breukelen GJP, Van Dijk KRA (2007) Use of covariates in randomized controlled trials. Journal of International Neuropsychology Society, 13, 903-904.

Timmermann L, Ploner M, Schmitz F, Baltissen R, Schnitzler A (2001) Differential Coding of Pain Intensity in the Human Primary and Secondary Somatosensory Cortex. Journal of Neurophysiology, 86, 1499-1503.

Valeriani M, de Tommaso M, Restuccia D, Le Pera D, Guido M, lannetti GD, et al. (2003) Reduced habituation to experimental pain in migraine patients: a CO2 laser evoked potential study. Pain, 105, 57-64.

Vallerand AH, Polomano RC (2000) The relationship of gender to pain. Pain Management Nursing, 1, 8-15.

Verbeke G, Molenberghs G, Thijs H, Lesaffre E, Kenward MG (2001) Sensitivity analysis for non-random dropout: a local influence approach. Biometrics, 57, 7-14.

Woestenburg JC, Verbaten MN, van Hees HH, Slangen JL (1983) Single trial ERP estimation in the frequency domain using Orthogonal Polynomial Trend Analyses (OPTA): estimation of individual habituation. Biological Psychology, 17, 173-191.

Zaslansky R, Sprecher E, Tenke CE, Hemli JA, Yarnitsky G (1996) The P300 in evoked potentials. Pain, 66, 39-49 




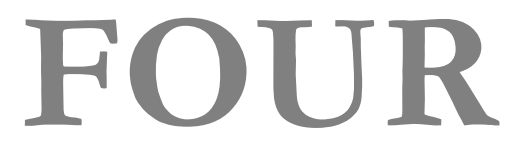

\title{
A challenge to the notion of a clear-cut association between Event-
}

\author{
Related Potentials and subjective pain ratings: An experimental study
}

\section{using multilevel analyses}

\section{Reference}

Vossen HGM, Van Breukelen GJP, Van Os J, Hermens H, Lousberg R (2009) A challenge to the notion of a clear-cut association between Event-Related Potentials and subjective pain ratings: An experimental study using multilevel analyses. Submitted for publication. 


\section{Abstract}

The association between Event-Related Potentials and subjective ratings of pain has frequently been demonstrated, usually through bivariate correlations. However, by using bivariate correlations it is not possible to study or correct for confounding factors. The aim of this study was to reinvestigate the association between Event-Related Potentials and the subjective experience, using a more extensive approach, namely multilevel analysis. In this manner, we were able to investigate and correct for effects of intensity and habituation. In this study, 85 healthy subjects received intracutaneous electrical pain stimuli with simultaneous EEG registration. Each subject was asked to rate the intensity of each stimulus on a Numeric Rating Scale (NRS). Multilevel analyses were performed in which maximum peak amplitudes (N1, P1, N2 and P3) served as independent variables to explain variance in NRS scores. Bivariate correlations, that were first conducted in this study by means of validation, corresponded (however less apparent) with findings from previous studies. The multilevel analyses revealed a strong association between the ERP measures (especially P1 and P3) and the NRS score. Furthermore, we found evidence that the intensity of the stimulus and habituation interact upon this association. The association between the ERP and subjective pain ratings increased when the intensity of the stimulus increased and that this association decreased over time. These results suggest that a direct translation from the pain-ERP to subjective experience is delicate and that factors such as stimulus intensity and habituation must be taken into account. 


\section{Introduction}

Event-Related Potentials (ERPs) are frequently used as a relatively objective means of measuring pain. Previous research has demonstrated positive correlations between the amplitude of specific peak components and the intensity of the pain stimulus (Stowell, 1977; Bromm, 1984). But what does an increase of a certain peak amplitude tell us about the amount of pain a subject feels?

One of the first studies that investigated the association between ERP and subjective pain ratings demonstrated that an increase in the N2 and P3 peak amplitude was accompanied by an increase in the subjective experience, using a Visual Analogue Scale (VAS) ( $r=0.67-0.77)$ (Harkins \& Chapman, 1978). These results were replicated by Chen (1979) and Garcia-Larrea (1997) who found a strong linear association ( $r=0.67$ and $r=0.41$ respectively) between the N2/P3 peak-to-peak amplitude and subjective painfulness. Miltner and colleagues (1987), however, could not demonstrate a similar association when investigating habituation of noxious stimuli. He found a significant decrease in peakto-peak amplitudes of the N150-P360 across trials but did not find a corresponding effect in the VAS. Miltner and co-workers (1987) thus suggested that the association between ERP amplitudes and subjective ratings might not be as strong as is previously claimed. Kanda and colleagues (2002) found that not only the N2 and the P3 component were associated with the subjective experience of pain but also a late positive component around $600 \mathrm{~ms}$ termed the 'intensity assessment-related potential' (IAP). The IAP occurs in paradigms where subjects are asked to subjectively rate the intensity of a stimulus. Kanda and colleagues (2002) found that IAP amplitude and latency was not influenced by intensity, thus suggesting that this component solely reflects the psychological processes of pain intensity assessment. Clearly, there appears to be a strong association between the amplitude of specific ERP components and the subjective experience of pain. However, closer investigation of the studies mentioned above generates some methodological concerns. First, in most ERP studies, single ERP trials are averaged over time to create a grand average. This procedure eliminates any unwanted 'noise' in the ERP, but in doing so it assumes that there is no difference between the repeated trials. This is problematic as single trials do likely differ from one another because of processes such as habituation. Moreover, averaging across trials eliminates all information about possible within-subject correlations between ERP and subjective pain. Such correlations can diverge substantially from the between-subject correlations (Van Breukelen, 2005). Furthermore, the studies described above, reported bivariate correlations in which no confounding factors such as stimulus intensity, habituation or age have been considered. 
The aim of this study was to reinvestigate the association between ERP and the subjective experience of pain. We first endeavoured to replicate the results of previous studies by calculating bivariate correlations followed by multilevel analyses including some relevant covariates. Following this, we tested interactions between stimulus intensity and ERP and between trial number and ERP as means of establishing the stability of the association between ERP measures and subjective ratings over trials and over different intensities.

\section{Methods}

\section{Subjects}

Eighty-five pain-free healthy subjects ( 33 males and 52 females) between the ages of 18 and 65 participated in this study. Exclusion criteria were the use of analgesics and/or the use of psychoactive drugs. Participants were provided with a $€ 25$.- reward.

\section{Stimuli}

The stimuli used in this study were electrical pulses of ten milliseconds duration. These were administered intracutaneously on the left middle finger (Bromm \& Meier, 1984). For each participant, five different intensities based on that participant's sensation and pain thresholds were administered. Of the five intensities, one was the pain threshold and the other four were defined relative to this pain threshold, namely $-50 \%,-25 \%,+25 \%$ and $+50 \%$ of the threshold range which was defined as the range between the sensation threshold and the pain threshold. The sensation threshold was determined by first administering stimuli at zero intensity and then gradually increasing the intensity until the stimuli were experienced consciously. Once experienced consciously (sensation threshold), stimuli were once again administered with an intensity that gradually increased from the sensation threshold until the stimuli were defined as painful by the participant (pain threshold). This procedure was repeated three times in order to generate a reliable measurement.

\section{Paradigm}

The stimuli were presented using a rating paradigm (Bromm, 1984). The paradigm consisted of 150 stimuli. The five intensities mentioned above were presented semi-randomly. The interstimulus interval (ISI) ranged from 9 to 11 seconds. Subjects were asked to rate the intensity of each stimulus on a Numeric Rating Scale (NRS) from 0 to 100 , whereby 0 is no sensation and 100 is the most excruciating 
pain imaginable. The first stimulus of the paradigm was set at the pain threshold. As a reference, subjects were instructed that this intensity was ' 60 '.

\section{EEG recording}

All EEG recordings were conducted in an electrically shielded and sound shielded cubicle $\left(3 * 4 \mathrm{~m}^{2}\right)$. $\mathrm{Ag} / \mathrm{AgCl}$ electrodes were placed on $\mathrm{Fz}, \mathrm{Cz}, \mathrm{Pz}, \mathrm{C} 3, \mathrm{C} 4, \mathrm{~T} 3$ and $\mathrm{T} 4$ using the international 10-20 system (Jasper, 1958). Impedances were kept below $5 \mathrm{k} \Omega$. A reference electrode was placed on each ear lobe. To control for possible vertical eye movements, an electro-oculogram (EOG) electrode was placed one centimetre under the midline of the right eye. A ground electrode was placed at Fpz. All electrodes were fixed using Ten $20^{\mathrm{tm}}$ conductive paste. Neuroscan 4.3 software was used for EEG recording.

\section{Procedure}

Before starting the experiment, subjects were informed about the purpose of the study and told that they would undergo EEG registration while receiving various intensities of electric stimuli, some of which would be painless and others that would be somewhat painful. After providing informed consent, EEG

electrodes were placed and the shock electrode was attached to the top of the left middle finger as described by Bromm and Meier (1984): a small opening in the upper layer of the skin was prepared using a dental gimlet. Care was taken that this procedure was not painful. In the prepared opening, a platinum electrode was placed and fixed with tape. The sensation and pain threshold were then determined and, once completed, the rating paradigm was initiated.

\section{Data reduction}

EEG was recorded with a $1000 \mathrm{~Hz}$ sampling rate using Neuroscan 4.3. The trials selected from the continuous EEG started $200 \mathrm{~ms}$ prior to the stimulus and ended $1500 \mathrm{~ms}$ post-stimulus. The data were filtered offline (bandpass $0-50 \mathrm{~Hz}$ ) and baseline correction took place. Trials with EOG activity exceeding $+75 \mathrm{~mA}$ or $-75 \mathrm{~mA}$ were excluded from the analyses. 


\section{Statistical analyses}

First, we endeavoured to replicate the findings of previous studies by calculating bivariate correlations between averaged NRS scores and averaged ERP measures. The ERP measures used in these correlations were as follows: 1) the maximum amplitudes of the N1-component (latency range: 20-55 msec); 2) the P1-component (latency range: 55-95 msec); 3) the N2-component (latency range: 95-145 msec); 4) the P3-component (latency range: 145-300 msec); and 5) the N2/P3 complex (slope between N2 and P3) (Including the sign i.e. at the N1-component and N2-component, the amplitude is $<0$ ). These components have been shown to be related to the processing of stimulus intensity (Kanda et al, 2002; Becker et al, 2000; Zaslansky et al, 1996; Bromm, 1984). We then conducted multilevel analyses with the NRS score per trial as dependent variable. Each ERP measure was split into a between-subject variable (mean peak) and a within-subject variable (peak minus the mean peak) in order to distinguish betweensubject correlations for ERP and subjective pain reporting from within-subject correlations. Furthermore, the following covariates were included in the model: intensity, trial linear, $_{\text {, }}$ rial $_{\text {quadratic }}$ [trial**], trial ${ }_{\text {inverse }}[1 /$ trial], age, gender, absolute sensation threshold, absolute pain threshold and intensity of the previous stimulus ${ }^{3}$. To note, trial $\left.\right|_{\text {linear }}$ was centred and runs from -74 to +74 , trial $\left.\right|_{\text {quadratic }}$

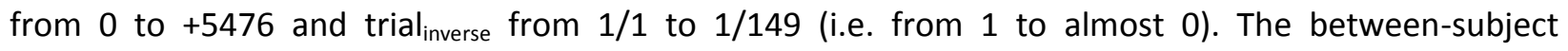
covariates, the within-subject factor intensity and ERP measures were all centred meaning that the sample mean was subtracted from each individual score (Van Breukelen \& Van Dijk, 2007). This was done so that we could interpret the intercept and prevent collinearity in models with many interaction terms. To accommodate differences between persons, the intercept and slopes of stimulus intensity and trial $_{\text {linear }}$ were allowed to vary randomly between persons. Finally, interactions between intensity and the ERP measures and between trial (linear, quadratic and inverse) and the ERP measures were included in the model.

Since the number of repeated measures was 150 (trials), the most general covariance structure that could be fitted was AR1 + measurement error (in SPSS, this is confusingly called ARMA1). This structure can handle both white noise (measurement error) and coloured noise (AR1). The goodness of fit of that covariance structure was significantly better than that of its competitors, namely compound symmetry (CS) and AR1. The random intercept and slopes were allowed to correlate by choosing for an unstructured $3 * 3$ covariance matrix for these effects.

\footnotetext{
${ }^{3}$ Based on results of a study which is currently under review, we decided to include a quadratic and inverse effect of trial in addition to the linear effect.
} 
As mentioned in section 2.3 , the NRS of the first trial was set at ' 60 '. Therefore there was no variance in NRS on this trial and thus this trial was left out of the analyses.

All statistical analyses were performed using SPSS 15.0. All p-values $\leq 0.001$ (two-tailed with Bonferroni correction for the number of cranial locations, namely seven, and components, namely four, each of which was split up into a between- and within-subject part) were considered as statistically significant.

\section{Results}

To get a general view of the NRS data, two figures are given that display the average NRS. Figure 1 gives the average NRS scores per trial. This shows a saw-tooth-like figure, which is partly caused by the semirandom presentation of five different intensities en partly due to unobserved within-subject state variations causing noise. The overall habituation of the NRS scores is a bit difficult to see, but when we look at the predicted habituation curve, it becomes clear that the NRS scores decrease over time (from about 50 to 30 ) and not just in a linear fashion. In figure 2, the average NRS scores per trial are displayed separately for the five different intensities. Again saw-tooth-like curves are presented, caused by withinsubject state variations and by variations in preceding trial intensities (a factor which will be seen to be related to NRS), but the habituation is more visible. This figure shows that the habituation is strongest in the first few trials.

\section{Association between subjective ratings and ERP: Correlations}

In order to replicate the results of previous studies, we calculated correlations between averaged ERP amplitudes and the averaged subjective rating of pain (NRS), using persons as the unit of analysis ( $N=$ 85). The results showed a significant and a marginally significant correlation of the $\mathrm{N} 2$ component at $\mathrm{Pz}$ and T3 (resp. $r=-0.346, p=0.001$ and $r=-0.233, p=0.033$ ).

The correlation between the N2/P3 peak-to-peak amplitude and the NRS was also calculated. At three cranial locations, namely Cz, Pz and C4 (resp. $r=0.237, p=0.030$ and $r=0.305, p=0.005$ and $r=0.221$, $p=0.045)$, did this slope correlate marginally significantly with the NRS. The ERP components, on which this correlation was found significant in previous studies, are equal to those found in the present study. However, in this study lower correlations were found. 
Association between subjective ratings and ERP: Multilevel analyses

In order to study the association between ERP measures and the subjective experience of pain, while controlling for relevant confounders, multilevel analyses were performed. In the initial model for the peak analyses, all components of all cranial locations and the covariates were included (except for the N2/P3 slope, to be included in section 3.4). ERP components that were not significant were consecutively deleted from the model until only Bonferroni-corrected significant ERP predictors remained. Table 1 displays the final model. This model showed significant effects of the P1-component (at Fz, T3 and T4), the N2-component (at Cz) and the P3-component (at Fz, Cz, T3 and T4). All these components were within-subject variables. Note that none of the between-subject ERP measures were significant. The directions of these effects were not the same for all these significant effects. The P3component at Fz and the P1-component at T3 and T4 had negative effect estimates, indicating that higher maximum peak amplitudes are related to lower subjective ratings. The other effects showed that stronger peak amplitudes are related to higher subjective ratings. Further, the results showed that

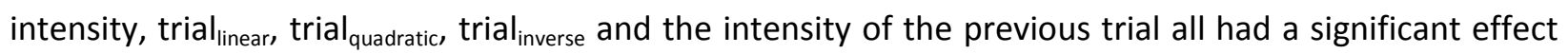
on the NRS scores. The effects of the between-subject covariates pain threshold, sensation threshold, age and gender were not significant. Furthermore, significant random between-subject variance was found with respect to the intercept and the effects (regression weights) of intensity and trial ${ }^{4}$. This implies person by intensity and person by trial number interactions, or stated differently, interpersonal differences in sensitivity to stimulus intensity and in rate of habituation.

\section{Interactions with intensity and trial}

The models described above, in which only significant ERP components were remaining, were supplemented by interactions between intensity and the ERP measures and by interactions between trial (linear, quadratic and inverse) and the ERP measures. The non-significant covariates (pain threshold, sensation threshold, age and gender) were excluded from the model, as were non-significant interactions, using alpha $=0.05$ for deletion and alpha $=0.001$ for conclusions. The results are displayed in Table 2. The analysis reveals a significant intensity*N2 effect (at Cz), a trial linear $^{*} \mathrm{~N} 2$ effect (at Cz) and a trial $_{\text {linear }}{ }^{*}$ P3 effect (on Cz). First, looking at interactions significant at the 0.001 level, the betas of those interactions with intensity imply that the relation of maximum peak amplitude of CZN2 with the NRS

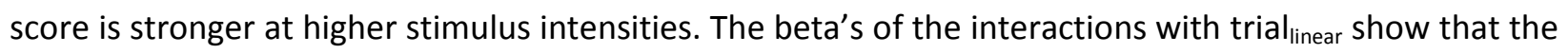

\footnotetext{
${ }^{4}$ The covariances accompanying these random between-subject variances were also statistically significant. Also, all AR1+ME noise parameters were significant (for details of the statistical model, see the appendix).
} 
relations of maximum peak amplitude $\mathrm{CzN} 2$ and $\mathrm{Cz} P 3$ with the subjective ratings apparently decrease over trials. Additional data analysis (not shown here) showed both ERP measures on NRS to diminish over trials and to be no longer significant at the last trial.

Looking next at marginally significant $(0.05>p>0.001)$ interactions, the peak analyses suggests an intensity*P3 effect (at $C z, p=0.005$ and $T 3, p=0.002$ ). What these results show, is that the association between subjective pain ratings and the amplitudes of specific components of the pain-ERP are dependent on the intensity of the stimulus and on time (trial number).

\section{Peak-to-peak component with multilevel analysis}

Lastly, we investigated the N2/P3 peak-to-peak, since this slope is often mentioned in the literature. We replaced the N2 and P3 peak amplitudes for each cranial location with two new predictors: 1) the sum of the two absolute peak amplitudes, and 2) the difference between the two absolute peak amplitudes. In this manner, it was possible to see whether the peak-to-peak component contains unique information (a significant difference measure) while maintaining the total ERP information in the model by supplementing the N2/P3 difference with the N2/P3 sum. With respect to N1 and P1 the model was as before and each ERP predictor was split up into a BS and WS term. For a clear comparison with the final model of Table 1 the model contained no interactions. Again, non-significant ERP components were consecutively deleted from the full model (all components of all cranial locations) until only significant ERP parameters remained. The results show that only the Czslope (difference) was significant and thus indicated that the N2/P3 peak-to-peak amplitude at Cz contains unique information (estimate $=0.038$, $\mathrm{SE}=0.007, \mathrm{p}<0.001)$ in the prediction of NRS. Thus, higher N2/P3 peak-to-peak amplitudes are accompanied by higher NRS.

\section{Discussion}

The main goal of this study was to investigate the association between Event-Related Potentials (ERP) and the subjective experience of pain in a comprehensive manner. Using multilevel analyses we were able to include single trial data and study the association between the ERP measures and subjective ratings not only between persons but also within persons.

By way of validation, we first calculated between-subject correlations for specific ERP components (N1, P1, N2, P3 and N2/P3slope) and the subjective ratings. Although the results of this study are comparable to those of previous studies, the present correlations were somewhat lower (correlations ranging from 
0.232 to 0.317$)$. A possible explanation for this is that in previous studies, trials were clustered by intensity and in the present study the intensities were mixed semi-randomly. Therefore it is possible that the present (rating) paradigm causes a larger variability in the (pain) ratings, leading to attenuation of correlations. However, an average of 150 trials is not expected to suffer much from attenuation due to measurement error.

Next, a multilevel analysis was performed on single trial data with the inclusion of possible influential covariates (stimulus intensity, trial number, pain threshold, sensation threshold, age and gender). The results showed that, independent of these covariates, especially the P1 and P3 components at $\mathrm{Fz}$ and $\mathrm{Cz}$ were highly significant predictors of NRS. As mentioned in the results section the direction of effect was not the same for all the significant ERP predictors. While most effect estimates (B) showed that larger peak amplitudes (of specific pain components) were related to higher NRS scores, some showed an opposite effect. These opposite effects were not in line with our a priori expectations. Since we corrected the result for multiple testing (Bonferroni correction) we cannot ignore this finding. Post hoc we explain this by the fact that different biopsychological processes (Feuerstien et al, 1987) are embedded in the pain-ERP and that these processes have different influences on the subjective experience of pain.

In a next step, we included interactions in the model in order to investigate whether the association between the ERP and subjective ratings was dependent on stimulus intensity or trial number. The results showed significant interactions of ERP with both stimulus intensity and trial number, all of these at $\mathrm{Cz}$ and most consistently concerned the P3 component. The interaction pattern (i.e. the B's) with intensity implies that the association between these ERP components and the subjective rating increases as the intensity of the stimulus increases. This finding was also reported by Chen and colleagues (1979). The interaction with trial number shows that the relation between specific components at $\mathrm{Cz}$ and the subjective pain ratings decreases as trial number increases.

The last step in the analyses concerned an inclusion of the N2/P3 peak-to-peak component. Since we not only calculated the difference in absolute peak amplitude between the N2 and the P3 component, but also their sum, we were able to determine if this slope contains unique information or if it is equal to including the two components separately in the model. The results show a genuine N2/P3 peak-topeak effect at $\mathrm{Cz}$. The marginally significant correlations we found earlier for the N2/P3 peak-to-peak at $\mathrm{Pz}$ and $\mathrm{C} 4$ were not replicated with the multilevel analyses, indicating that these did not contain unique 'slope' information. 
In the analyses described above, only within-subject ERP variables were significant. It is possible that the between-subject ERP variables were not significant because of low power (the effective sample size for between-subject effects being the number of persons and not the number of trials), and/or because of a restriction of range (since stimulus intensities were geared to each person's sensory and pain threshold which may have reduced between-subject variance in ERP or NRS). Another potential explanation is quite simply that there is no between-subject association for ERP and NRS. However, the regression weights of the between-subject ERP measures in the initial models were larger than those of the withinsubject measures. The same held for standardized regression weights. This makes the low power explanation more likely than the explanation that there is simply no between-subject association for ERP and NRS.

With respect to the covariates intensity, trial (linear, quadratic and inverse) and intensity of the previous trial, we found, as expected, highly significant effects. The intensity effect means that stronger intensities elicit higher NRS scores. The three significant trial effects (linear, quadratic and inverse) represent three time patterns. The trial ${ }_{\text {inverse }}$ effect has been described by Milne and colleagues (1999) and signifies a strong habituation in only the first few trials. The trial linear $_{\text {effect has also often been }}$ observed in experimental research (Miltner et al, 1987; Arntz \& Lousberg, 1990) and represents a

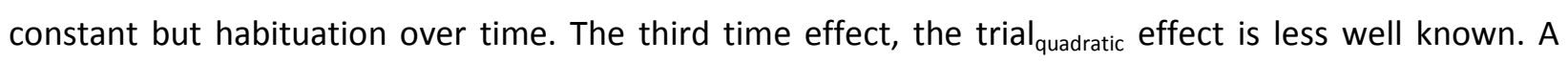
quadratic function (a parabolic function) is characterized by a decrease, which is followed by an increase. Although the quadratic effect was significant in the analyses, no actual dishabituating effect was found. Only a decrease in maximum peak amplitude was found. This is probably because the number of stimuli was not large enough. Aside from the intensity and trial effects, the intensity of the previous trial was also an important predictor of NRS. This implies that subjects based their NRS of the actual stimulus partly on the intensity of the previous trial.

A point of concern in this study is the generalizability of the present results from the laboratory to clinical practice. Pain elicited in a controlled experimental setting, as was the case in our study, differs from clinical pain in sensation, duration, controllability and meaning. Another point of concern is that, in this study, only healthy pain-free subjects participated.

We believe that the results of this study provide evidences that the link between ERP measures and NRS is far more complex than previously thought. When using Event-Related Potentials as a relatively objective measurement of pain, it is important to realize that single trial data should be used since also intensity, trial number and intensity of the previous trial are strong predictors of subjective pain ratings. Furthermore, the association between ERP measures and subjective ratings appears to depend on 
stimulus intensity and trial number (i.e. habituation), as the finding of several interactions indicates. Future research that seeks to replicate the present results and generalize them to other populations (e.g. pain patients), other pain stimuli (e.g. C02 laser stimuli or paradigms (e.g. oddball), is required to gain further insight in the complex association between subjective pain reports and Event-Related Potentials. 


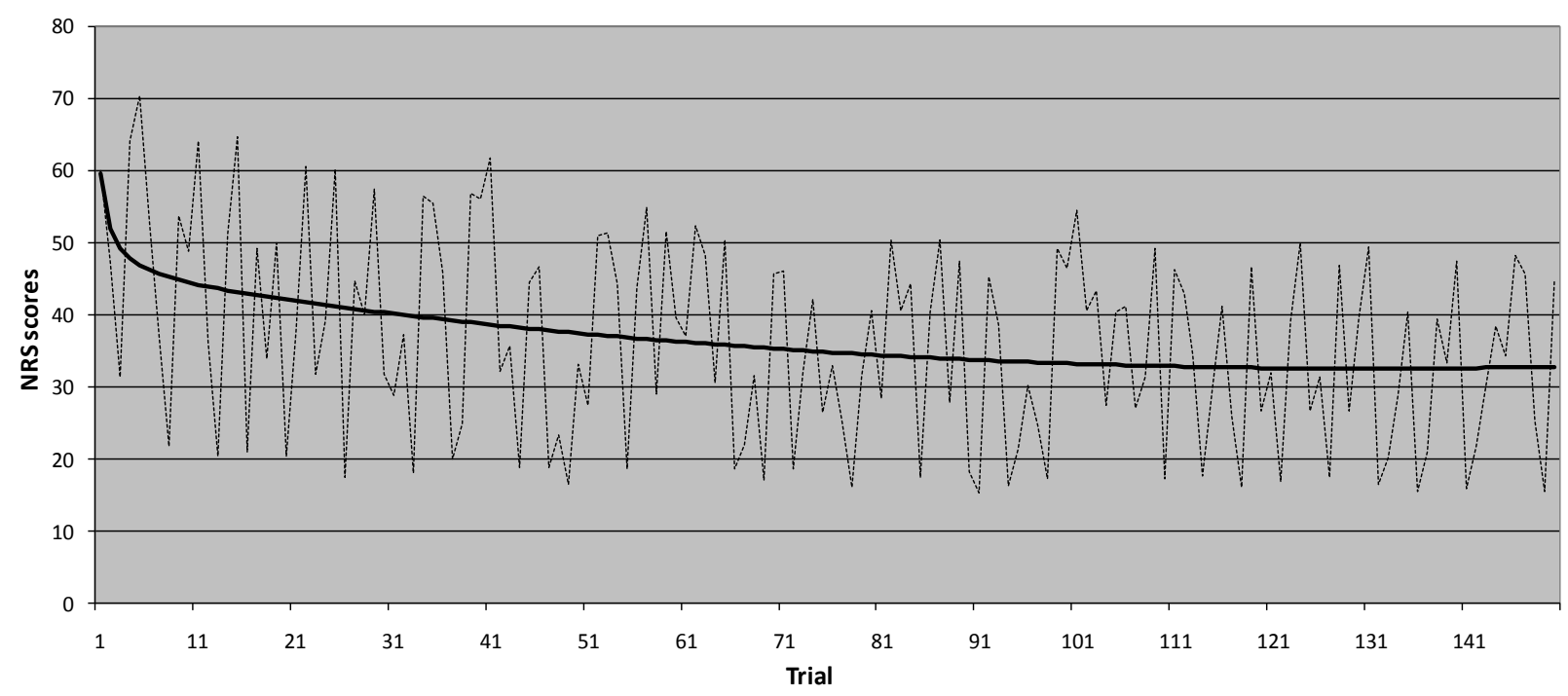

Figure 1 NRS scores per trial. The thicker solid line represents the predicted curve based on the model with only the three trial effects as predictors.

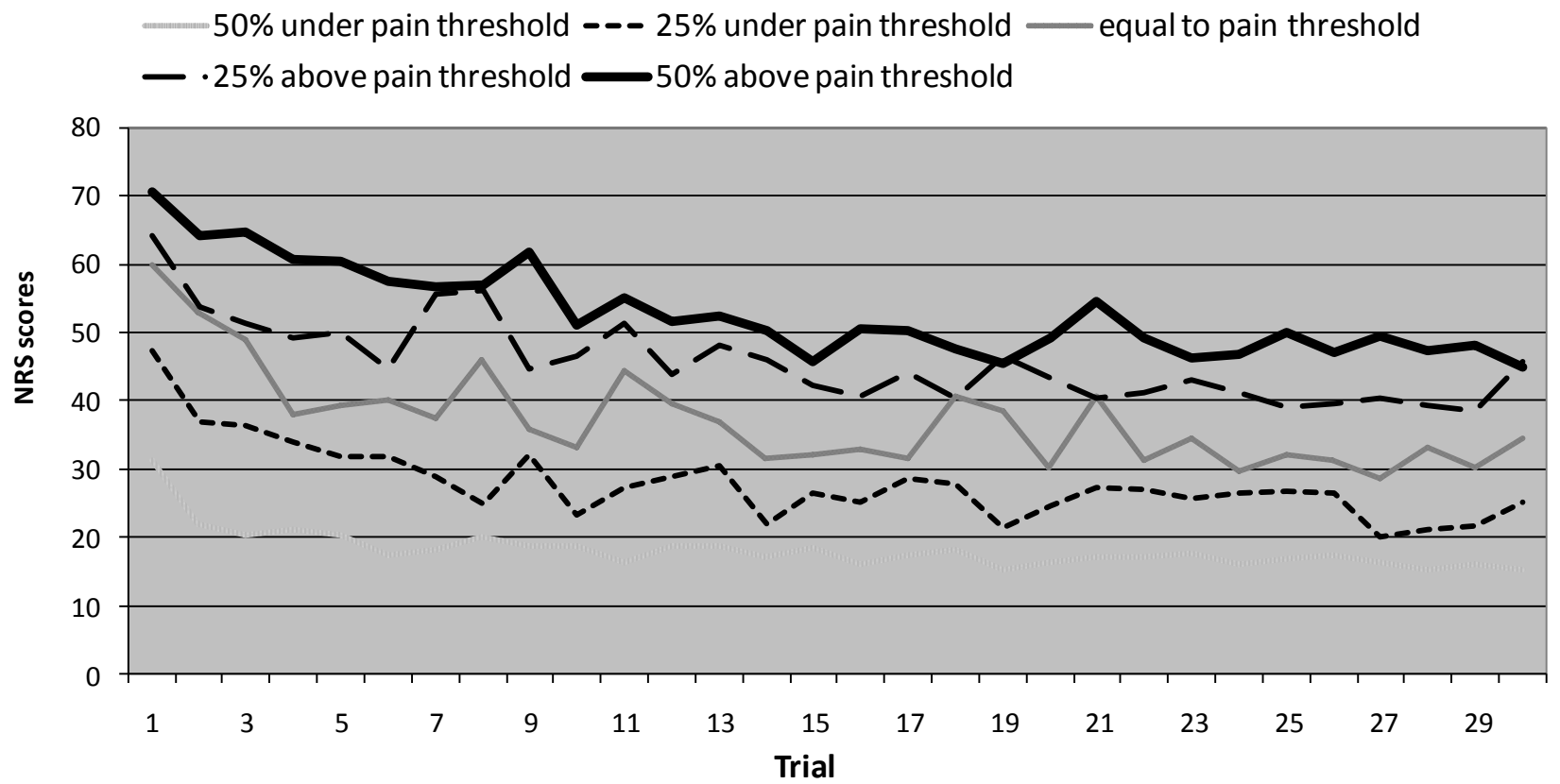

Figure 2 NRS scores per intensity and trial 
Table 1 Table 1 Estimates, standard errors and two-tailed level of significance are given for the fixed and random parameters. WS $=$ within-subject. Finally, the series of residuals $e_{i j}$ of person i was a sum of white noise (measurement error) plus colored noise (autoregressive of order 1), with parameter estimates: $\sigma_{e}^{2}=106.86, \rho=0.731$ and $\varphi=0.305$. For details of this noise structure, see the appendix.

\begin{tabular}{|c|c|c|c|}
\hline Parameter & Estimate (B) & Std. Error (SE) & Sig. (p) \\
\hline \multicolumn{4}{|l|}{ Fixed } \\
\hline Intercept & 35.8539 & 1.6658 & $<0.001$ \\
\hline Intensity & 9.3696 & .4346 & $<0.001$ \\
\hline Trial $_{\text {linear }}$ & -.0695 & .0098 & $<0.001$ \\
\hline Trial $_{\text {quadratic }}$ & .0006 & .0001 & $<0.001$ \\
\hline Trial $_{\text {inverse }}$ & 12.3340 & 1.5040 & $<0.001$ \\
\hline Absolute pain threshold & 1.5986 & 1.7641 & .368 \\
\hline Absolute sensation threshold & -1.6002 & 11.2386 & .887 \\
\hline Age & .0311 & .0923 & .737 \\
\hline Gender & -.2733 & 1.4162 & .848 \\
\hline Intensity of the previous trial & 1.5893 & .0642 & $<0.001$ \\
\hline FzP1 (WS) & .0484 & .0107 & $<0.001$ \\
\hline CzN2 (WS) & -.0336 & .0074 & $<0.001$ \\
\hline FzP3 (WS) & -.0318 & .0079 & $<0.001$ \\
\hline CzP3 (WS) & .0318 & .0075 & $<0.001$ \\
\hline T3P1 (WS) & -.0580 & .0142 & $<0.001$ \\
\hline T4P1 (WS) & -.0533 & .0141 & $<0.001$ \\
\hline T3P3 (WS) & .0442 & .0119 & $<0.001$ \\
\hline T4P3 (WS) & .0442 & .0099 & $<0.001$ \\
\hline \multicolumn{4}{|l|}{ Random } \\
\hline Intercept variance & 207.9981 & 34.6196 & $<0.001$ \\
\hline Intensity slope variance & 14.8754 & 2.4077 & $<0.001$ \\
\hline Trial $_{\text {linear }}$ slope variance & 0.0059 & 0.001 & $<0.001$ \\
\hline
\end{tabular}


Table 2 Multilevel model with maximum peak parameters and interactions with intensity and trial. Estimates, standard errors and two-tailed level of significance are given for the fixed and random parameters. WS= within-subject. $\sigma_{e}^{2}=$ 117.638, $\rho=0.602$ and $\varphi=0.182$.

\begin{tabular}{|c|c|c|c|}
\hline Parameter & Estimate & Std. Error & Sig. \\
\hline \multicolumn{4}{|l|}{ Fixed } \\
\hline Intercept & 35.7650 & 1.6113 & $<0.001$ \\
\hline Intensity & 9.2671 & .4473 & $<0.001$ \\
\hline Trial $_{\text {linear }}$ & -.0605 & .0094 & $<0.001$ \\
\hline Trial $_{\text {quadratic }}$ & .0004 & $7.8356 \mathrm{E}-5$ &.$<0.001$ \\
\hline Trial $_{\text {inverse }}$ & 17.6184 & 1.4366 & $<0.001$ \\
\hline Intensity of the previous trial & 1.5947 & .0721 & $<0.001$ \\
\hline FzP1 (WS) & .0646 & .0106 & $<0.001$ \\
\hline CzN2 (WS) & -.0338 & .0074 & $<0.001$ \\
\hline FzP3 (WS) & -.0440 & .0078 & $<0.001$ \\
\hline CzP3 (WS) & .0273 & .0075 & $<0.001$ \\
\hline T3P1 (WS) & -.0757 & .0140 & $<0.001$ \\
\hline T4P1 (WS) & -.0541 & .0140 & $<0.001$ \\
\hline T3P3 (WS) & .0554 & .0118 & $<0.001$ \\
\hline T4P3 (WS) & .0464 & .0099 & $<0.001$ \\
\hline CzN2 (WS)*intensity & -.0263 & .0053 & $<0.001$ \\
\hline CzP3(WS)*intensity & .0140 & .0050 & .005 \\
\hline T3P3(WS)*intensity & .0194 & .0062 & .002 \\
\hline $\mathrm{CzN}_{2}(\mathrm{WS}) *$ trial $_{\text {linear }}$ & .0006 & .0002 & $<0.001$ \\
\hline CzP3(WS)* trial $\left.\right|_{\text {linear }}$ & -.0008 & .0002 & $<0.001$ \\
\hline \multicolumn{4}{|l|}{ Random } \\
\hline Intercept variance & 206.1606 & 33.5825 & $<0.001$ \\
\hline Intensity slope variance & 15.6020 & 2.8828 & $<0.001$ \\
\hline Trial $_{\text {linear }}$ slope variance & 0.0061 & 0.0010 & $<0.001$ \\
\hline
\end{tabular}




\section{References}

Arntz A, Lousberg R (1990) The effects of underestimated pain and their relationship to habituation. Behavior Research Therapy, 28, 15-28.

Becker DE, Haley DW, Urena VM, Yingling CD (2000) Pain measurement with evoked potentials: combination of subjective ratings, randomized intensities, and long interstimulus intervals procedures a P300-like confound. Pain, 84, 37-47.

Bromm, B, Meier W (1984) The intracutaneous stimulus: a new pain model for algesimetric studies. Methods and Findings in Experimental Clinical Pharmacology, 6, 405-410.

Bromm B (1984) Pain-related components in the cerebral potential: experimental and multivariate statistical approaches. In Pain measurement in man: Neurophysiological correlates of pain (Ed. Bromm B), pp. $257-290$. Elsevier: Amsterdam.

Chen CAN, Chapman CR, Harkins SW (1979) Brain potentials are functional correlates of induced pain in man. Pain, 6, 365-374.

Feurestein M, Papciak AS, Hoon PE (1987) Biobehavioral mechanisms of chronic low back pain. Clinical Psychology Review, 7, 233-273.

Garcia-Larrea L, Peyron R, Laurent B, Mauguiere F (1997) Association and dissociation between laser-evoke potentials and pain perception. Neuroreport, 8, 3785-3789.

Harkins SW, Chapman CR (1978) Cerebral evoked responses to noxious dental stimulation: relationship to subjective pain report. Psychophysiology, 15, 248-252.

Jasper $\mathrm{HH}$ (1958) The ten-twenty electrode system of the International Federation. Journal of Electroencephalography Clinical Neurophysiology, 20, 371-375.

Kanda M, Matsuhashi M, Sawamoto N, Oga T, Mima T, Nagamine T, Shibasaki H (2002) Cortical potentials related to assessment of pain intensity with visual analogue scale (VAS). Clinical Neurophysiology, 113, 1013-1024.

Milne RJ, Kay NE, Irwin RJ (1999) Habituation to repeated painful and non-painful cutaneous stimuli: A quantitative psychophysiological study. Experimental Brain Research, 187, 438-444.

Miltner W, Larbig W, Braun C (1987) Habituation of subjective rating and event related potentials to painful intracutaneous electrical stimulation of the skin. Journal of Psychophysiology, 1, 221-228.

Stowell H (1977) Cerebral slow waves related to the perception of pain in man. Brain Research Bulletin, 2, 23-30.

Van Breukelen GJP (2005) Psychometric modelling of response speed and accuracy with mixed and conditional regression. Psychometrika, 70, 359-376.

Van Breukelen GJP, Van Dijk KRA (2007) Use of covariates in randomized controlled trials. Journal of International Neuropsychology Society, 13, 903-904.

Zaslansky R, Sprecher E, Tenke CE, Hemli JA, Yarnitsky G (1996) The P300 in evoked potentials. Pain, 66, 39-49 




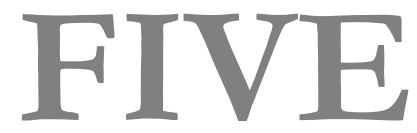

\title{
The predictive value of pain Event-related potentials for clinical
}

\author{
experience of pain
}

\section{Reference}

Vossen HGM, Van Os J, Hermens H, Lousberg R (2009) The predictive value of pain Event-Related Potentials for clinical experience of pain. Accepted in Journal of Integrative Neuroscience 


\section{Abstract}

Event-Related Potentials have been found to be related to subjective experience of experimental pain. But how are they related to the subjective experience of clinical pain? The current study investigated the predictive value of the pain ERP for the subjective experience of clinical pain. Event-Related Potentials in response to experimental pain were measured in 75 chronic low back pain sufferers. In addition a twoweek registration was done of the amount of pain they experienced in daily life. The results demonstrate that the N2-component at $\mathrm{Cz}$ and $\mathrm{C} 4$ of painERP (contralateral to the side of the stimulation) were significant predictors of clinical pain, even stronger predictors that the accompanying subjective ratings of experimental pain. Thus, it seems promising to use Event-Related Potentials as a more objective measure to make predictions about a person's likely pain experience in daily life. 


\section{Introduction}

Many experimental studies have been conducted in order to examine individual differences in the experience of pain. An important advantage of experimental laboratory studies is that the environment can be controlled and thus more specific conclusions can be drawn. However, an important issue in experimental laboratory research is the difficulty in generalizing the results to clinical practice. In experimental work, the intensity, duration and location of a pain stimulus typically are under experimental control. However, it is difficult to translate these pain responses to the clinical experience of pain. Furthermore, quantitative psychophysiological measures such as the peak amplitudes of EventRelated Potentials are even more difficult to translate to subjective pain experience. Although research has demonstrated that ERPs can serve as a relatively objective measurement of pain compared to subjective pain ratings (Stowell, 1977; Bromm, 1984; Becker et al, 1993) and evidence has been provided that specific peak components of the ERP significantly correlate with subjective ratings of pain (Garcia-Larrea et al, 1997; Kanda et al, 2002), the question remains what exactly ERP measures tell us about the amount of pain that people experience in daily life. Can the pain-ERP be used as a predictor for clinical pain?

To our knowledge, no studies have been performed that attempted to answer these questions. However, there have been some studies in that demonstrated the predictive value of ERPs for other clinical outcomes. Kemp and colleagues (2006) found that, in combination with personality and cognitive factors, the left-lateralized P150 could predict the severity of non-clinical depression. Also, specific components of the ERP have been found to predict awakening in coma patients (Daltrozzo et al, 2006). Furthermore, selected ERP time segments across different electrodes have proven to contain signal features that discriminate Alzheimer patients from control subjects with high sensitivity and specificity (Benvenuto et al, 2002).

The current study was designed to measure pain responses in both a laboratory setting and in daily life, in a sample of patients with chronic low back pain. The aim of the study was twofold. First, the relation between subjective ratings of experimental pain and subjective ratings of clinical pain was examined. Second, it was investigated to what degree cortical processing of experimental pain can predict clinical pain in daily life. The hypotheses were that i) a positive association exists between subjective ratings of experimental pain and clinical pain and ii) persons showing stronger pain processing in the experimental setting show higher pain ratings in daily life. 


\section{Methods}

Approval has been obtained from the medical ethics committee of the Academic Hospital Maastricht, on January, 6th, 2005. All subjects gave their verbal and written informed consent prior to the experiment.

\section{Subjects}

Seventy-seven subjects with chronic non-specific low back pain were included. Chronic low back pain was specifically chosen since this is a highly prevalent chronic pain problem which is a clear recognizable complaint for patients. The latter was especially important because the patients were drawn from the general population (via advertisements distributed door to door). They were required to have low back pain for at least six months with no other interfering pain complaints. The low back pain complaints concerned non-malignant chronic pain. Furthermore, they did not use any analgesics or psychoactive drugs.

\section{Stimuli}

The stimuli used in the experimental part of the study were electrical pulses of ten milliseconds duration. These were administered intracutaneously on the left middle finger. For each participant, five different intensities based on that participant's sensation and pain thresholds were administered. Of the five intensities, one was the pain threshold and the other four were defined relative to this pain threshold, namely $-50 \%,-25 \%,+25 \%$ and $+50 \%$ of the threshold range which was defined as the range between the sensation threshold and the pain threshold. The sensation threshold was determined by first administering stimuli at zero intensity and then gradually increasing the intensity until the stimuli were experienced consciously. Once experienced consciously (sensation threshold), stimuli were once again administered with an intensity that gradually increased from the sensation threshold until the stimuli were defined as painful by the participant (pain threshold). This procedure was repeated three times in order to generate a more reliable measurement.

\section{Rating paradigm}

The stimuli, described above, were presented using a rating paradigm (Bromm, \& Meier, 1984). The paradigm consisted of 150 stimuli. The five intensities mentioned above were presented semi-randomly. The inter-stimulus interval (ISI) ranged from 9 to 11 seconds. Subjects were asked to rate the intensity of 
each stimulus on a Numeric Rating Scale (NRS) from 0 to 100 , whereby 0 is no sensation and 100 is the most excruciating pain imaginable. The first stimulus of the paradigm was set at the pain threshold. As a reference, subjects were instructed that this intensity was ' 60 '.

\section{EEG recording}

All EEG recordings were conducted in an electrically- and sound-shielded cubicle $\left(3 * 4 \mathrm{~m}^{2}\right) . \mathrm{Ag} / \mathrm{AgCl}$ electrodes were placed on $\mathrm{Fz}, \mathrm{Cz}, \mathrm{Pz}, \mathrm{C} 3, \mathrm{C} 4, \mathrm{~T} 3$ and T4 using the international 10-20 system (Jaspers, 1958). Impedances were kept below $5 \mathrm{k} \Omega$. A reference electrode was placed on each ear lobe. In order to control for possible vertical eye movements, an electro-oculogram (EOG) electrode was placed 1 centimetre under the midline of the right eye. A ground electrode was placed at Fpz. All electrodes were fixed using 10-20 conductive paste. Neuroscan 4.3 software was used for EEG recording.

\section{Personality}

Subjects were asked to complete the NEO Personality Inventory. The NEO-PI-R is based on a five-factor model of personality. The five personality factors measured are Neuroticism, Extraversion, Openness, Agreeableness and Conscientiousness. Validity and reliability measures of the NEO-PI-R are adequate (Hoekstra et al, 1996).

\section{Booklets}

In order to measure pain in daily life, subjects completed a daily questionnaire containing 4 questions concerning their pain complaints, during a two week period. Subjects were instructed to answer the questions before bedtime. The 4 questions were:

How much pain do you have at this moment? (pain)

How is your mood at this moment? (mood)

How much physical and mental effort did your activities cost today? (effort)

How much stress did you experience today? (stress)

These questions where assessed with a Visual Analogue Scale measuring $100 \mathrm{~mm}$. Validity and reliability measures of the NEO-PI-R are adequate (Hoekstra et al, 1996). 


\section{Procedure}

Before starting the experiment, subjects were informed about the purpose of the study. Subjects were told that they would undergo EEG-registration while they received electric shocks. After signing the informed consent form, subjects were asked to fill in the NEO-PI-R. Then, EEG electrodes were placed and the shock electrode was attached to the top of the left middle finger as described by Bromm and Meier (1984): a small opening in the upper layer of the skin was prepared using a dental gimlet. Care was taken that this procedure was not painful. In the prepared opening, a platinum electrode was placed and fixed with tape. Next, the sensation and pain threshold were determined and after that the Rating paradigm was initiated. After the experiment subjects returned home and started filling in the first booklet the day after the experiment.

\section{ERPs}

EEG was recorded with $1000 \mathrm{~Hz}$ sampling rate, using Neuroscan 4.3 software. Trials were selected from the continuous EEG, from 200 ms prior to the stimulus until 1500 ms post-stimulus. Data was offline filtered (bandpass $0-50 \mathrm{~Hz}$ ) and baseline-corrected. Trials with EOG activity exceeding $+75 \mathrm{~mA}$ and $-75 \mathrm{~mA}$ were excluded from the analyses.

\section{Statistical analyses}

Linear regressions were performed in which the mean pain score of the booklets rated over 14 days was used as dependent variable. The analyses were performed in three steps. First, significant covariates were determined. We hypothesized that the following covariates were of potential influence on the mean pain score: age, gender, mood, stress, effort (all measured from the booklets), duration of pain complaints and personality (five main factors: Neuroticism, Extraversion, Openness, Conscientiousness and Altruism). Second, the mean rating in response to experimental pain stimuli (NRS) was added as predictor of clinical pain. Third, in addition to the significant covariates, mean ERP peak amplitudes of the following components: N1 (20-55msec), P1 (56-95msec), N2 (96-145msec), P2 (146-300msec) were tested as predictors of clinical pain. These components have been shown to be associated with the processing of stimulus intensity (Kanda et al, 2002; Becker et al, 2000; Zaslansky et al, 1996; Bromm, 1984). Only the mean ERP measures of the strongest intensity were used in the analyses. 
All statistical analyses were performed using SPSS 16.0 software. P-values $\leq 0.05$ were considered statistically significant. For the analyses concerning the ERP measures, the p-value was corrected for multiple testing $(0.05 / 3=0.017)$.

\section{Results}

Seventy-seven subjects (35 men and 42 women) with chronic low back-pain were included in the analyses. The mean pain score collected over 14 days was computed based on valid ratings only (sum/ (14-number of missing values)). More than $83 \%$ of the subjects did not have any missing data. The mean pain score was normally distributed with an overall mean of $38.9(S D=16.3)$. The mean age was 40.3 years $(S D=15.2)$. Mean duration of the low back-pain complaints was 12.2 years $(S D=11.9)$. Figure 1 shows the grand average of the response to the strongest pain intensity ( $50 \%$ above the pain threshold) at $\mathrm{Cz}$.

First, covariates which significantly predicted the mean pain score were identified. A regression analysis with the mean clinical pain score as dependent variable and the list of covariates as independent variables (see method section), showed that only stress (as reported in the booklets) and conscientiousness (one of the NEO-PI-R scales) were significant predictors. Stress was positively associated with the clinical pain score $(B=0.58, S E=0.13, p<0.001)$, indicating that a higher stress score was associated with higher clinical pain scores. Conscientiousness was also positively associated with clinical pain ( $B=0.29, S E=0.10, p=0.003$ ). Subjects who were more conscientious reported higher levels of clinical pain. This model explained $30 \%$ of the total variance of the mean pain score. The other covariates did not significantly predict clinical pain score (all p-values $>0.15$ ).

In order to test the first hypothesis, a regression with stress, conscientiousness and the mean NRS as predictors was tested. This model demonstrated that subjective ratings of experimental pain stimuli significantly predicted subsequent ratings of clinical pain $(B=-0.23, S E=0.11, p=0.042)$. The Beta was negative, indicating that individuals with lower ratings of experimental pain reported stronger pain in daily-life. This model explained $34 \%$ of the total variance of the mean pain score. Thus, hypothesis 1 was rejected.

For the second hypothesis, the ERP measures were added instead of the subjective ratings of experimental pain. Separate models were tested for each ERP component (4), and locations were clustered: the central midline locations $(\mathrm{Fz}, \mathrm{Cz}, \mathrm{Pz})$, the lateral locations $(\mathrm{C} 3, \mathrm{C} 4, \mathrm{~T} 3, \mathrm{~T} 4)$ and the sensory 
motor cortex locations $(C 3, C z, C 4)$. The results of the series of regression analyses yielded one specific significant model with significant ERP predictors. This model consisted of the psychological predictors in combination with the $\mathrm{N} 2$-component at $\mathrm{Cz}$ and $\mathrm{C} 4$ (table 1 ). This model explained $37 \%$ of the total variance of the mean pain score, an increase of $7 \%$ compared to the model with only the covariates. The two N2-components had directionally opposite B estimates, the B of the N2-component at $\mathrm{Cz}$ being negative and the $\mathrm{B}$ of the N2-component at $\mathrm{C} 4$ being positive. Further analysis revealed that the two N2components were only statistically significant when they were both in the model. This model explained $39 \%$ of the total variance of the mean pain score.

Finally, the model with significant ERP measures was extended with the mean NRS score (table 2). This model revealed that the mean NRS was no longer a significant predictor of clinical pain when corrected for the cortical processing of experimental stimuli. The N2-components of Cz and C4, however, did remain statistically significant, indicating their effect was not reducible to other predictors.

\section{Discussion}

The aim of the current study was to investigate whether Event-Related Potentials in response to experimental noxious stimuli predicted the level of clinical pain in daily life. The results demonstrated that this was the case.

Since multiple factors influence the subjective experience of clinical pain, significant covariates were determined before the hypotheses were tested. Stress, which was also measured in daily life, was the covariate with the strongest. Higher stress scores were associated with higher clinical pain ratings. This finding is in accordance with findings of previous studies (Labbé et al, 1997; Aslaksen \& Flaten, 2008). Furthermore, the personality trait conscientiousness was also positively associated with ratings of clinical pain. Conscientiousness is a personality trait which is characterized by self discipline, carefulness, deliberation and need for achievement (Hoekstra et al, 1996). Subjects who were more conscientious reported more clinical pain. This is in agreement, to a degree, with an earlier study by Farman and colleagues (2008), who demonstrated that chronic pain patients showed higher scores on the conscientiousness scale compared to pain free controls. All other hypothesized covariates were not associated with clinical pain ratings.

Subsequently, the association between subjective ratings of experimental pain (NRS) and ratings of clinical pain was tested. The results showed that, in combination with the two covariates, higher ratings of clinical pain were associated with lower ratings of experimental pain. This was not as hypothesized: 
subjective response to experimental pain and clinical pain co-vary in opposite rather than similar directions. A possible post-hoc explanation may be that persons experiencing higher levels of pain in daily life are "de-sensitized" to experimental nociceptive stimuli.

Next, the association between cortical processing of experimental pain and ratings of clinical pain was analysed. Independent of the covariates stress and conscientiousness, the N2-component at Cz and C4 were significant predictors of the mean rating of clinical pain. The N2-component at Cz was negatively associated with clinical pain whereas the N2-component at C4 was positively associated with clinical pain. The fact that these components were only significant in the presence of each other suggests 'suppression'. Each of these two ERP measures may explain a small but unique part of the mean pain variance, and co-depend on each other in the model. These directionally opposite associations are interesting since it was expected that a stronger N2-component was associated with more pain in dailylife (the more negative the N2-component the higher the mean pain score). This only holds true for the $\mathrm{N} 2$-component at $\mathrm{Cz}$. The opposite was observed for the N2-component at $\mathrm{C} 4$, where more negative ERP amplitudes correspond with lower mean pain ratings. This suggests an association corresponding to pain reduction for the N2-component at $\mathrm{C} 4$ and one suggesting increase in pain for the N2-component at $\mathrm{Cz}$. The phenomenon of inhibitory and excitatory factors influencing the pain experience has been described earlier (Wall, 1964).

Finally, analyses in which mean NRS as well as the ERP measures were included revealed that the ERP measures remained significant predictors whereas the subjective NRS did not. This finding suggests that ERP measures may be useful for prediction of chronic pain over-and-above subjective ratings, possibly because Event-Related Potentials are less vulnerable to bias and are multidimensional measures (measuring more aspects of the processing of pain).

This study has some limitations. First, pain in daily life was measured only once a day, at a fixed time. Perhaps multiple random measurements during the day, for example using momentary assessment technology (Myin-Germeys et al, 2009), provides more detailed information about pain and its course during the day. Second, a possible important covariate of daily pain ratings, which was not measured in this study, is the presence of a partner or spouse during the research interview. Research has revealed a significant pain augmenting influence of solicitous responses of spouses (Lousberg et al, 1992; Schwartz \& Kraft, 1999). Including this covariate in the analyses could perhaps explain more error variance of mean pain score and have a positive effect on the explanatory capacity of the ERP measures. Finally, we performed 12 regression analyses in order to test the hypotheses related to ERP measures. P-values were only corrected for the clusters of cranial locations. Perhaps this does not lower the type I error 
enough. However, a strict Bonferroni correction increases the type II error substantially, which could result in "throwing the child out with the bathwater" (Perneger, 1998).

This study demonstrates that the pain-ERP measured in an experimental laboratory setting has predictive value for clinical pain in daily life. Although the contribution of the ERP measures to the explanation of the mean pain variance may be considered relatively small (7\%), the fact that a significant contribution to pain in daily life up to two weeks later could be demonstrated may be considered remarkable. Thus, the two ERP components in questions may be investigated further for use as an objective measure to make predictions about a person's likely pain experience in daily life. 


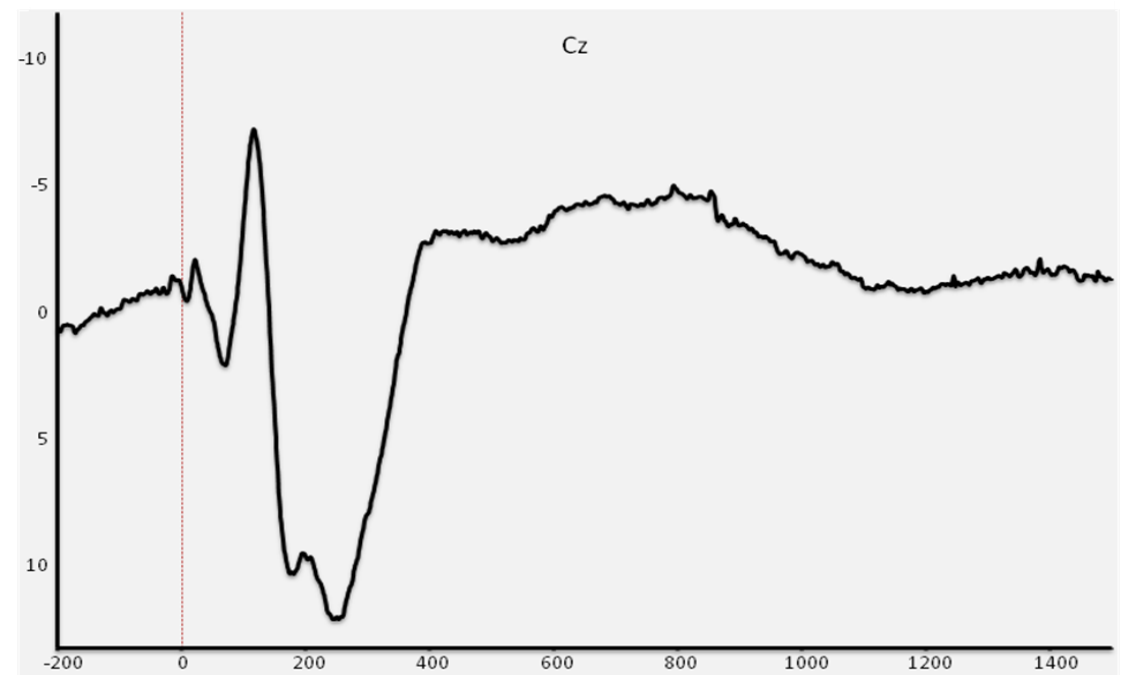

Figure 1 Grand Average of the strongest pain intensity ( $50 \%$ above the pain threshold) at $\mathrm{Cz}$. The $\mathrm{Y}$ axis represent amplitude in $\mu \mathrm{V}$ and the the $\mathrm{x}$-axis represents time in milliseconds.

Table 1 Regression coefficients of the predictors of subjective rating of clinical pain

\begin{tabular}{|lrrr|}
\hline Model & \multicolumn{2}{l}{ Unstandardized Coefficients } & \multicolumn{1}{l|}{ Sig. } \\
& \multicolumn{3}{c}{ Std. Error } \\
\hline stress & .668 & .126 & .000 \\
conscientiousness & .256 & .095 & .009 \\
CzN2 & -1.472 & .508 & .005 \\
C4N2 & 1.750 & .675 & .012 \\
\hline
\end{tabular}

Table 2 Regression coefficients of the predictors of subjective rating of clinical pain

\begin{tabular}{|c|c|c|c|}
\hline \multirow[t]{2}{*}{ Model } & \multicolumn{2}{|c|}{ Unstandardized Coefficients } & \multirow[t]{2}{*}{ Sig. } \\
\hline & B & Std. Error & \\
\hline stress & .671 & .125 & .000 \\
\hline conscientiousness & .270 & .094 & .005 \\
\hline $\mathrm{CzN} 2$ & -1.348 & .508 & .010 \\
\hline C4N2 & 1.599 & .673 & .020 \\
\hline mean NRS & -.179 & .111 & .110 \\
\hline
\end{tabular}




\section{References}

Aslaksen PM, Flaten MA (2008) The roles of physiological and subjective stress in the effectiveness of a placebo on experimentally induced pain. Psychosomatic Medice, 70, 811-818.

Bromm, B, Meier W (1984) The intracutaneous stimulus: a new pain model for algesimetric studies. Methods and Findings in Experimental Clinical Pharmacology, 6, 405-410.

Bromm B (1984) Pain-related components in the cerebral potential: experimental and multivariate statistical approaches. In Pain measurement in man: Neurophysiological correlates of pain (Ed. Bromm B), pp. 257-290. Elsevier: Amsterdam.

Becker DE, Haley DW, Urena VM, Yingling CD (2000) Pain measurement with evoked potentials: combination of subjective ratings, randomized intensities, and long interstimulus intervals procedures a P300-like confound. Pain, 84, 37-47.

Benvenuto J, Jin Y, Casale M, Lynch G, Granger R (2002) Identification of diagnostic evoked response potentials segments in Alzheimer's disease. Experimental Neurology, 176, 269-276.

Daltrozzo J, Wioland N, Mutschler V, Kotchoubey B (2007) Predicting coma and other low responsive patient's outcome using event-related potentials: a meta-analysis. Clinical Neurophysiology, 118, 606-614.

Farman A, Somi MH, Sarami F, Farhang S (2008) Five personality dimensions in patients with irritable bowel syndrome. Neuropsychiatric Disease and Treatment, 4, 959-962.

Garcia-Larrea L, Peyron R, Laurent B, Mauguiere F (1997) Association and dissociation between laser-evoke potentials and pain perception. Neuroreport, 8, 3785-3789.

Hegerl U, Gallinat J, Juckel G (2001) Event-Related Potentials: Do they reflect central serotonergic neurotransmission and do they predict clinical response to serotonin agonists? Journal of Affective Disorders, 62, 93-100.

Hoekstra HA, Ormel J, de Fruyt F (1996) NEO-PI-R - NEO FFI. Big five persoonlijkheidsvragenlijsten. Handleiding. Lisse: Swets \& Zeitlinger.

Jasper HH (1958) The ten-twenty electrode system of the International Federation. Journal of Electroencephalography Clinical Neurophysiology, 20, 371-375.

Kanda M, Matsuhashi M, Sawamoto N, Oga T, Mima T, Nagamine T, Shibasaki H (2002) Cortical potentials related to assessment of pain intensity with visual analogue scale (VAS). Clinical Neurophysiology, 113, 1013-1024.

Kemp AH, Hopkinson PJ, Stephan BC, Clark R, Gordon E, Bryant RA, Williams LM (2006) Predicting severity of nonclinical depression: preliminary findings using an integrated approach. Journal of Integrated Neuroscience, 5, 89110.

Labbé EE, Murphy L, O'Brien C (1997) Psychological factors and prediction of headaches in college adults. Headache, 37, 1-5.

Lousberg R, Schmidt AJ, Groenman NH (1992) The relationship between spouse solicitousness and pain behavior: searching for more experimental evidence. Pain, 51, 75-79. 
Myin-Germeys I, Oorschot M, Collip D, Lataster J, Delespaul P, van Os J (2009) Experience sampling research in psychopathology: opening the black-box of daily life. Psychological Medicine, 12, 1-15

Perneger TV (1998) What's wrong with the Bonferroni Adjustments. British Medical Journal, 316, 1236-1238.

Schwartz L, Kraft GH (1999) The role of spouse responses to disability and family environment in multiple sclerosis. American Journal of Physical Medicine \& Rehabilitation, 78, 525-532.

Stowell H (1977) Cerebral slow waves related to the perception of pain in man. Brain Research Bulletin, 2, 23-30.

Wall PD (1964) Presynaptic control of impulses at the first central synapse in the cutaneous pathway. Journal of Psychophysiology, 188, 403-423.

Zaslansky R, Sprecher E, Tenke CE, Hemli JA, Yarnitsky G (1996) The P300 in evoked potentials. Pain, 66, 39-49. 



\title{
SIX
}

\section{Cortical differences in habituation to experimental pain between}

\author{
chronic low back pain sufferers and pain-free controls: the role of
}

depressive symptoms

\section{Reference}

Vossen HGM, Van Breukelen, GJP, Hermens H, Van Os J, Lousberg R (2009) Cortical differences in habituation to experimental pain between chronic low back pain sufferers and pain-free controls: the role of depressive symptoms. Submitted for publication. 


\section{Abstract}

A deficit in habituation to painful stimuli is often proposed as an explanation for the chronicification of pain. Only a limited number of studies has investigated whether chronic pain patients have a deficit in habituation in comparison to pain-free controls. The aim of this study was to investigate difference in habituation of cortical pain processing between chronic pain patients and pain-free controls. Seventyeight subjects with chronic low back pain complaints and seventy-nine pain-free controls received electrical pain stimuli. Event-Related Potentials served as outcome pain measures. Differences in habituation between the two groups were investigated with multilevel random regression analysis. Furthermore, the influence of depressive symptoms on any difference in habituation was investigated. An interaction between group and trial number was found, indicating that the maximum peak amplitudes decreased over trials in pain-free controls but not patients with chronic pain. In addition, a significant three-way interaction between group, trial number and depressive symptoms was found, indicating even lower habituation in the chronic pain group with higher level of depressive symptoms. Subjects with long lasting low back pain may not habituate to painful stimuli. Furthermore, depressive psychopathology may further increase sensitivity to painful stimuli in this group. 


\section{Introduction}

Low back pain is a frequently occurring pain complaint. In most cases, low back pain resolves spontaneously within a short period of time. Sometimes, however, the pain persists and turns chronic pain. One of the most important questions in pain research is why some people develop chronic pain complaints whereas most others do not. Several mechanisms have been proposed to explain such individual differences, for example based on cognition and coping styles (Woby et al, 2007; Campbell en Edwards, 2009). However, one explanation for the chronification of pain which to date remains underresearched involves the hypothesis of a deficit in habituation of painful sensations. Habituation is a decrease in behavioural response to a stimulus that is repeatedly presented (Thompson \& Spencer, 1966). It is thought that chronic pain patients may present with an inability to habituate to painful experiences, resulting in persistent pain. Studies investigating this topic have reported mixed results. Brandt \& Schmidt, (1987) studied pain tolerance in 8 successive cold pressure tasks in chronic low back pain patients and pain-free controls. They reported that the pain-free control group could be divided into a subgroup which habituated over trials and a subgroup which sensitized over the trials. The chronic pain group, however, neither habituated nor sensitized over time. Arntz and colleagues (1990) found that the amount of habituation of pain intensity ratings after 20 successive painful electric shocks was equal for both the chronic low back pain group and pain-free controls. Another study from Peters and co-workers (1989) found habituation in the control group but not in the group of chronic low back pain patients, thus confirming the results of Brandt \& Schmidt, (1987). More recently, Valeriani and colleagues (2003), measuring Event-Related Potentials (ERP) in response to painful $\mathrm{CO}_{2}$ laser stimulation, found reduced habituation of ERP amplitudes in migraine sufferers compared to pain-free controls. Tommaso (2005) also studied habituation of ERP amplitudes in response to $\mathrm{CO}_{2}$ laser stimulation and found that migraine sufferers did not show habituation, whereas pain-free controls did. Recently, a study by Smith and co-workers (2008) reported differences in habituation of subjective pain ratings between women suffering from fibromyalgia and pain-free female controls. They found that the women with fibromyalgia habituated in response to repeated heat stimuli at a much lower rate than pain-free female controls.

The studies described above used different chronic pain populations, pain stimuli and outcome measures and this might explain the differences in results. Nevertheless, the studies overall are suggestive of a deficit in habituation in chronic pain patients (as opposed to pain-free controls), but it is 
not clear whether chronic pain patients have an inability to habituate (qualitative difference) or that they merely habituate less than pain-free controls (quantitative difference).

This study examines in more detail the difference in habituation of experimental pain stimuli between chronic pain patients and pain-free controls. Similar to Valeriani and co-workers (2003) and Tommaso (2005), Event-Related Potentials were used as outcome measure. Essential in research on habituation is to study differences in the responses to repeated stimuli. In pain-ERP research, this means that differences in cortical processing between repetitive pain stimuli should be the focus of analysis. This is often a problem, given the large amount of stimuli presented. Therefore, averages are usually computed to bring this large number back to a small number of block averages. In this averaging process, however, within-subject information is lost, while in the case of habituation this represents the primary material for analysis. Using multilevel analysis techniques, it is possible to include single-trial data and thus obtain all within-subject information. Smith and colleagues (2008) also used the multilevel analysis technique to investigate habituation. However, in their study, no Event-Related Potentials were recorded.

Therefore, the aim of this study was to investigate whether people experiencing chronic pain have a reduced habituation of experimental pain stimuli in comparison to pain-free controls. Furthermore, guided by previous literature (Tang et al, 2008; Young et al, 2008), and previous results that show differences in depressive symptoms between chronic pain sufferers and pain-free controls, the influence of depressive symptoms on reduced habituation was considered. The following hypotheses were tested: (i) Compared to pain-free controls, a reduced or absent habituation of the pain-ERP in the chronic pain group is expected (i.e. a group*trial number interaction); (ii) This interaction is expected to be dependent on the level of depressive symptoms (i.e. a group*trial number*depressive symptoms interaction).

\section{Methods}

\section{Subjects}

Seventy-eight subjects with low back pain complaints were included in the sampling frame. The subjects had to have low back pain for at least six months with no other interfering pain complaints. All subjects had had physical therapy one time or another, but none were in treatment at the time of the experiment. Furthermore, seventy-nine pain-free subjects participated in the study. The age ranged 
from 18 to 65 years. General exclusion criteria were the use of analgesics and psychoactive drugs. Participation was rewarded with $€ 25$,-.

\section{Stimuli}

Stimuli were electrical pulses of 10 milliseconds duration, which were administered intracutaneously on the top of the left middle finger. The pain threshold was determined by gradually increasing the intensity of the stimulus, starting at zero intensity. Subjects had to indicate at which intensity the stimulus became painful. This procedure was repeated three times for a more reliable measurement.

\section{Habituation paradigm}

The paradigm consisted of 20 stimuli of identical intensity, equal to the pain threshold. The Inter stimulus Interval (ISI) was kept at between 9 and 11 seconds.

\section{Psychological assessment}

Subjects were asked to complete the Beck Depression Inventory (BDI). This self-administered inventory measures depressive symptoms and attitudes with 21 questions which can be rated 0 to 3 in terms of intensity. The BDI is a valid and reliable inventory (Beck et al, 1988).

\section{EEG recording}

All EEG recordings were conducted in an electrically- and sound-shielded cubicle $\left(3 * 4 \mathrm{~m}^{2}\right) . \mathrm{Ag} / \mathrm{AgCl}$ electrodes were placed on $\mathrm{Fz}, \mathrm{Cz}, \mathrm{Pz}, \mathrm{C} 3, \mathrm{C} 4, \mathrm{~T} 3$ and T4 using the international 10-20 system (Jasper, 1958). Impedances were kept below $5 \mathrm{k} \Omega$. A reference electrode was placed on each ear lobe. In order to control for possible vertical eye movements, an electro-oculogram (EOG) electrode was placed $1 \mathrm{~cm}$ under the midline of the right eye. A ground electrode was placed at Fpz. All electrodes were fixed using 10-20 conductive paste. Neuroscan 4.3 software was used for EEG recording.

\section{Procedure}

Before starting the experiment, subjects were informed about the purpose of the study. Subjects were told that they would undergo EEG-registration while they received electric shocks. They were not informed that all stimuli had the same intensity. After signing the informed consent form, EEG 
electrodes were placed and the shock electrode was attached to the top of the left middle finger as described by Bromm and Meier (1984). Next, the pain threshold was determined and after that, the habituation paradigm initiated.

\section{Data reduction}

EEG was recorded with $1000 \mathrm{~Hz}$ sampling rate, using Neuroscan 4.3 software. Trials were selected from the continuous EEG, from $200 \mathrm{~ms}$ prior to the stimulus until $1500 \mathrm{~ms}$ post-stimulus. Data was offline filtered (bandpass $0-50 \mathrm{~Hz}$ ) and baseline corrected. Trials with EOG activity exceeding $+75 \mathrm{~mA}$ and -75 $\mathrm{mA}$ were excluded from the analyses.

\section{Statistical analyses}

SPSS 16.0 was used to perform the analyses. A multilevel model for the first hypothesis was performed with the following maximum peak components: N1 (20-55 msec), P1 (56-95 msec), N2 (96-145 msec), P2 (146-300 msec), as dependent variable. Trial number, pain threshold, age, gender and group served as independent variables. Trial number was entered in two ways: in its raw form to accommodate a linear trend, and in its inverse form (1/trial number) to accommodate strong change in the first trials and levelling off at later trials. This was done based on results of an earlier study (Vossen et al, submitted).

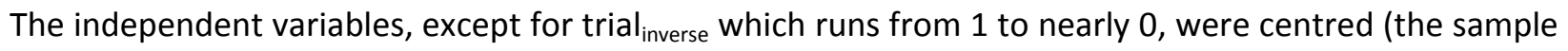
mean was subtracted from each individual score) (Van Breukelen, \& Van Dijk, 2007).

In order to study the difference in habituation between the chronic low back pain suffers and pain-free controls, the interaction terms group*trial ${ }_{\text {linear }}$ and group* trial $_{\text {inverse }}$ were included. For the second hypothesis, a measure for depressive complaints (BDI) as main effect, as well as the two- and three-way interactions with group and trial (linear and inverse) was included. Within-subject trial-to-trial variation was assumed to follow an autoregressive AR1 process.

For both hypotheses, i.e. the group by trial interaction and the depression by group by trial interaction, all p-values $\leq 0.007$ (two-tailed with Bonferroni correction for the number of cranial locations, namely seven) were considered as statistically significant. In order not to be overly conservative, effects were considered threshold significant and reported for $p$-values between 0.01 and 0.05 . 


\section{Results}

\section{Descriptive statistics}

The groups only differed significantly with respect to BDI scores, with the chronic low back pain sufferers having higher scores compared to the pain-free controls (Table 1). Since depressive symptomatology was considered a moderator, it was not included as a confounder (second hypothesis).

\section{Reduced or absent habituation of the pain-ERP in the chronic pain group compared to controls}

In order to test the first hypothesis, analyses were performed in which the maximum peak amplitude of a certain component (4) at a certain cranial location (7) served as the dependent variable. Trial number, pain threshold, age, gender and group served as independent variables. This meant that a total of 28 analyses were performed for this hypothesis. The interaction between trial and group was of main interest. A statistically significant interaction between group and trial $\left.\right|_{\text {linear }}$ was found on the P2component of $\mathrm{C} 4(\mathrm{~B}=0.15, \mathrm{SE}=0.042, \mathrm{p}<0.001)$ and $\mathrm{T} 4(\mathrm{~B}=0.12, \mathrm{SE}=0.04, \mathrm{p}=0.001)$. Similar, although of sub-Bonferroni significance, effects (also with trial linear $_{\text {) }}$ were found on the N2-component of $P z(B=-0.078, S E=0.04, p=0.025)$ and the $P 2$-component of $T 3(B=0.04, S E=0.02, p=0.017)$. Figure 1a shows the observed differences over trials between the two groups, based on the raw data of the P2component of C4. Visual inspection of figure 1a demonstrates that the pain-free control group starts off with slightly higher amplitudes than the chronic pain group. Furthermore, the amplitude of the pain-free control group seems to slowly decrease over time, while the amplitude of the chronic pain group overall stays constant (except for a decrease from trial 1 to 2 ). Figure $1 \mathrm{~b}$ shows the predicted difference based on the estimates of the analyses. This figure shows that the peak amplitude of pain-free controls is predicted to decrease more over trials compared to that of the chronic pain sufferers. No significant interactions between group and trial inverse $_{\text {were found. }}$

In sum, the results support the first hypothesis in that chronic pain sufferers show no habituation of the pain-ERP, while the pain free controls do.

\section{The group by trial interaction is moderated by level of depressive symptoms.}

In order to investigate the role of depressive symptoms on the difference in habituation between

chronic pain sufferers and pain-free controls, three-way interactions (group*trial linear $^{*}$ depression and group*trial $_{\text {inverse }}{ }^{*}$ depression) were added to the model described in the previous section. One three-way 
interaction was statistically significant: group*trial inverse $\mathrm{BDI}$ on the $\mathrm{N2}$-component of $\mathrm{Pz}(\mathrm{B}=0.71, \mathrm{SE}=$ $0.26, p=0.007)$. Three were found marginally significant: group*trial $\left.\right|_{\text {inverse* }} \mathrm{BDI}$ on the N1-component of $\mathrm{Fz}(\mathrm{B}=0.64, \mathrm{SE}=0.25, \mathrm{p}=0.008)$ and the $\mathrm{N} 2$-component of $\mathrm{Fz}(\mathrm{B}=0.78, \mathrm{SE}=0.30, \mathrm{p}=0.01)$ and $\mathrm{C} 4(\mathrm{~B}=$ $0.56, \mathrm{SE}=0.25, \mathrm{p}=0.027$ ). Figures $2 \mathrm{a}$ and $2 \mathrm{~b}$ show the predicted direction of effects based on the estimates from the analyses of the N2 component of Pz. Figure 2a shows that when depression scores are low, the maximum peak amplitude of both groups increases over the first few trials and then decreases slowly over time. When depression scores are high (figure $2 b$ ), however, the maximum peak amplitude of the chronic pain group seems to increase slightly (become more negative). The peak amplitude of the pain-free controls decreases (become less negative) in a clear non-linear (inverse) manner. The contrast between the pain-free group and the chronic pain group is most evident in the first few trials.

These results confirm the hypothesis that depression influences the difference in habituation between the chronic low back pain sufferers and pain-free controls. Subjects experiencing chronic pain and reporting depressive symptoms show a lack of habituation, at least at the N2 component of Pz.

\section{Discussion}

This study examined the hypothesis that people with chronic pain complaints habituate less or not at all, at the cortical level, to painful stimuli in comparison to pain-free controls. Furthermore, the moderating influence of depressive symptoms on this difference in habituation was investigated. Multilevel analysis enables inclusion of single-trial data of Event-Related Potentials and thus study habituation to painful stimuli in a detailed manner.

The first hypothesis examined whether chronic low back pain sufferers habituated less to painful stimuli

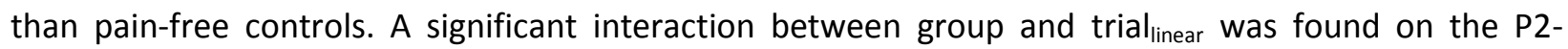
component of $\mathrm{C} 4$ and T4. This effect showed that people experiencing chronic low back pain appeared to habituate to a much lesser degree than pain-free controls. A directionally similar interaction was found, albeit at a marginal level of significance, on the P2-component of T3 and the N2-component of Pz. These results are in line with the study by Smith and colleagues (2008) discussed earlier. The N2 and P2-components are thought to be generated by neurons in the cingulate cortex, which is part of the limbic system (Tarkka and Treede, 1993; Valeriani et al, 1996). This system is important for the emotional components of pain. 
For the second hypothesis, a measure of depressive symptoms (BDI) was added to the model of the first hypothesis. The results revealed significant three-way interactions between BDI score, group and trial $_{\text {inverse }}$ on the N2-component of Pz and C4 and marginally significant three-way interactions on the N1component and N2-component of Fz. These results suggest that in the presence of depressive symptoms, the pain-free group habituates to the stimuli while the chronic pain group does not. An explanation for this effect might be that the etiological pathway of depressive symptoms in pain-free controls differs from that in the chronic pain group. The depressive symptoms of the chronic pain group may be secondary to the exposure of long lasting pain, generating a vicious circle of increasing sensitivity (vigilance) to pain which was picked up in the experimental condition. The depressive symptoms in the pain-free group are unlikely associated with chronic pain and therefore form no basis for sensitization to painful stimuli. Another explanation might be that a third factor, for instance a genetic factor, influences both depressive symptoms as well as pain complaints. In addition, table 1 displays significantly higher depressive symptomatology for the chronic pain patients compared to the pain free controls. For this reason, the observed moderating effect of depressive symptoms may in part also constitute a mediating effect, something which cannot be separated statistically.

The study has a number of limitations. First, since no subjective pain ratings were measured no statements on the difference in habituation between the two groups at a given level of subjective (pain) experience can be made. Furthermore, the chronic pain sufferers were recruited through advertisements. Since the subjects were not referred by a medical doctor or physical therapist, no objective diagnostic information was available. It is possible that unknown medical factors influenced the results. However, by choosing this type of recruitment, specificity of pain complaints was lost, but generalizability was gained. Clinical patient populations represent only the tip of the iceberg when it comes to the total number of people experiencing pain in everyday life. This study aimed to address the large group of people with chronic pain that are not currently in the medical circuit.

In conclusion, with the use of Event-Related Potentials and multilevel analysis, the present study indicates that habituation to painful experimental stimuli may be different depending on the presence of (i) chronic pain and (ii) depression. In contrast to pain-free controls, chronic pain sufferers, especially in the presence of depressive complaints, may show less to no habituation. Prospective studies, with pain-free subjects or acute pain patients, are required to investigate whether this deficit in habituation is a causal mechanism in the development of chronic pain. 
Table 1 Sociodemographic information

\begin{tabular}{|lll|}
\hline & $\begin{array}{l}\text { Chronic low back pain sufferers } \\
(\mathrm{N}=78)\end{array}$ & $\begin{array}{l}\text { Pain free controls } \\
(\mathrm{N}=79)\end{array}$ \\
\hline Gender $\quad$ male & $39(50 \%)$ & $31(39.24 \%)$ \\
& $39(50 \%)$ & $48(60.76 \%)$ \\
Age (years) & $40.25(15.28)$ & $35.68(14.57)$ \\
Absolute pain threshold (mV) & $1.15(0.96)$ & $0.96(0.68)$ \\
BDI & $7.03(4.57)$ & $3.05(3.17)^{*}$ \\
\hline
\end{tabular}

* Significant with an independent sample t-test $(t=16.23, p<0.001)$

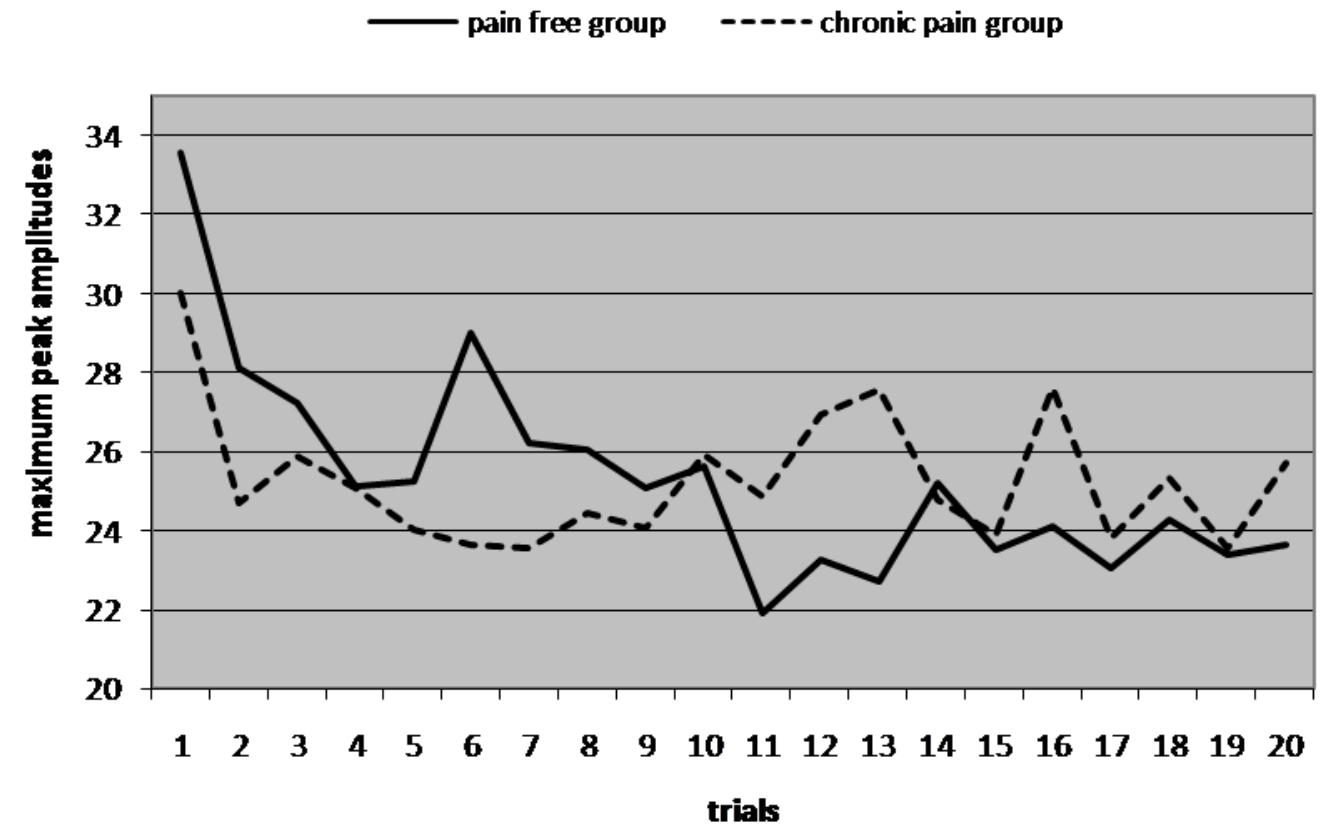

Figure 1a Group amplitudes over trials (P2-component of C4) 


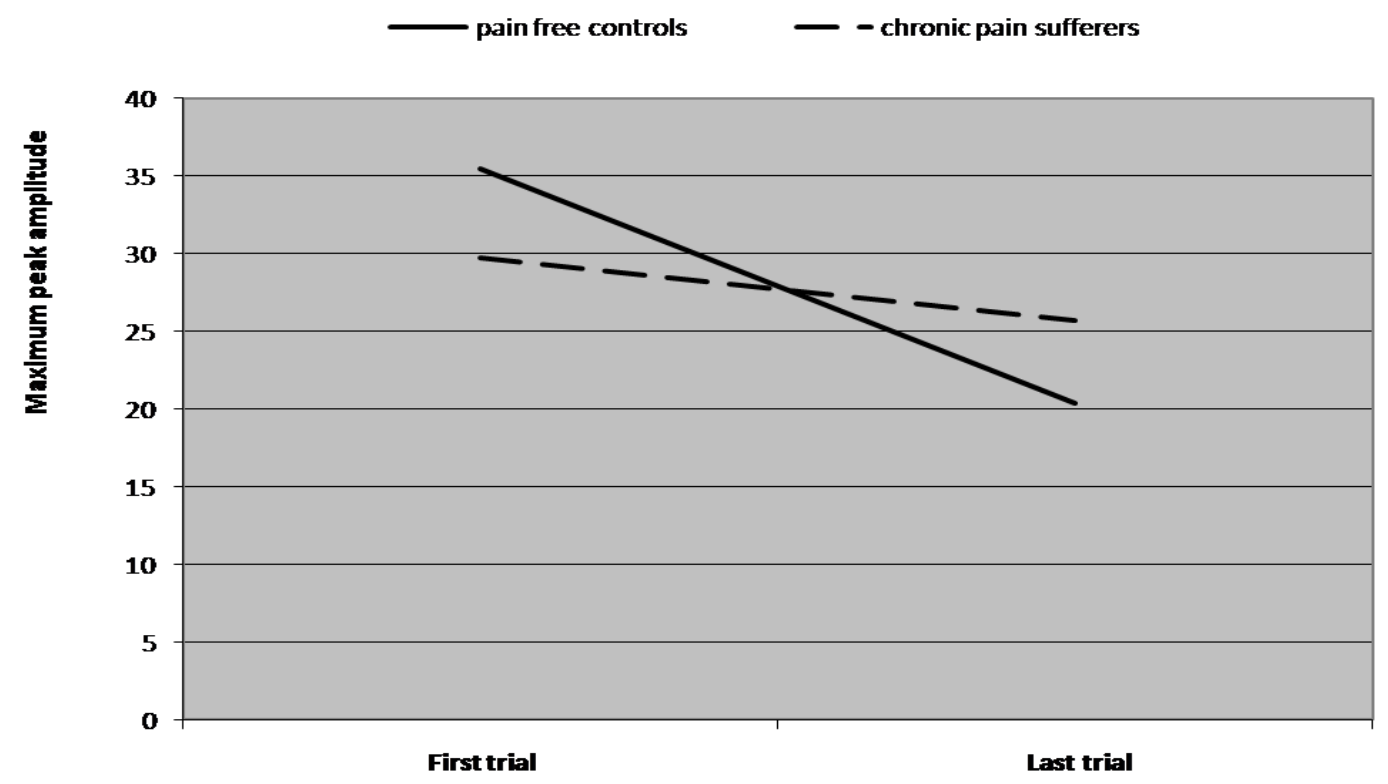

Figure 1b Group amplitudes over trials (P2-component of C4)

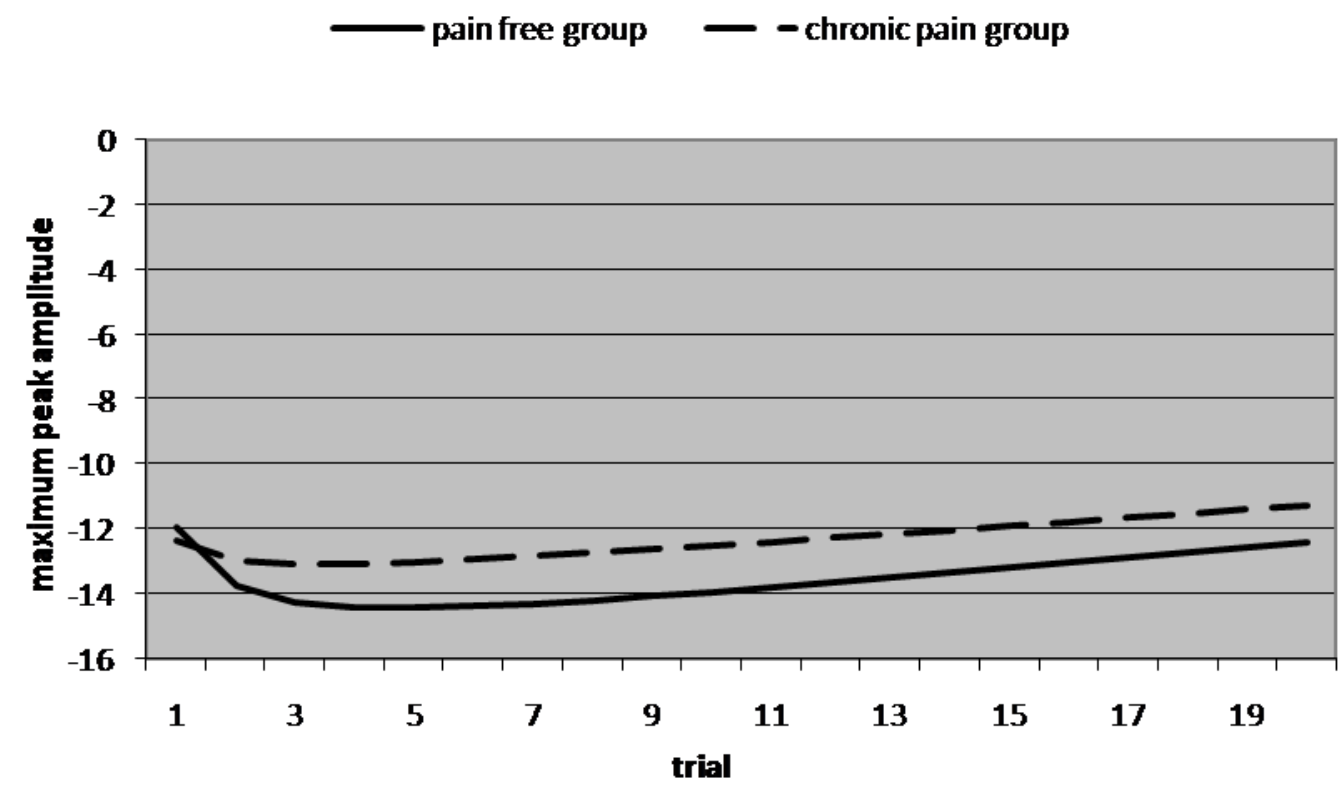

Figure 2a Predicted direction of effect when depressive symptoms are low (N2-component of Pz) 


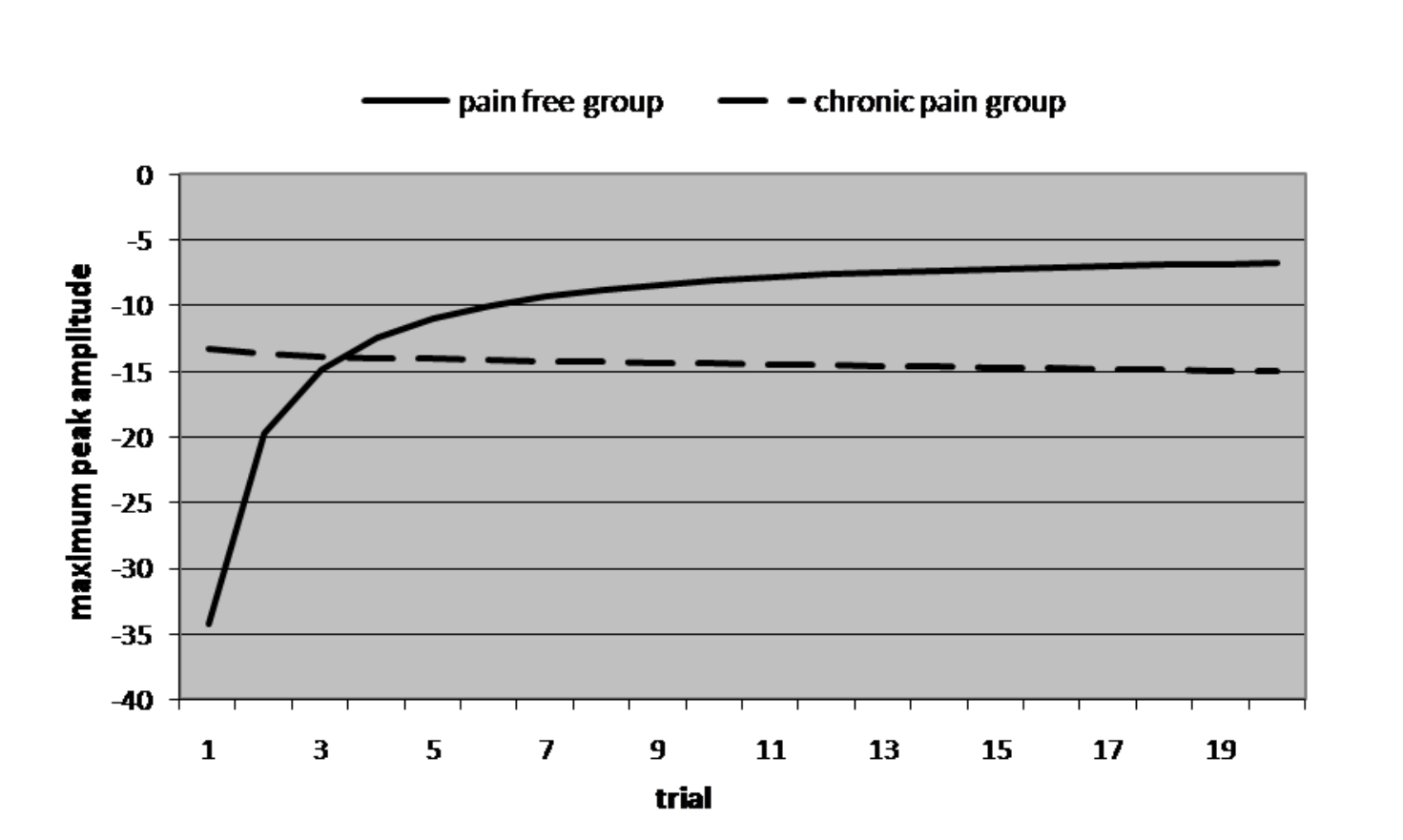

Figure $2 \mathrm{~b}$ Predicted direction of effect when depressive symptoms are high (N2-component of Pz) 


\section{The full multilevel model:}

$Y_{t i}=\beta_{0}+\beta_{1}$ trial linear $+\beta_{2}$ trial $_{\text {inverse }}+\beta_{3}$ gender $+\beta_{4}$ age $+\beta_{5}$ absolute sensation threshold $+\beta_{6}$ group $+\beta_{7} B D I+\beta_{8}$ trial linear ${ }_{\text {group }}+\beta_{9}$ trial $_{\text {inverse }} *$ group $+\beta_{10}$ trial $_{\text {linear }} B D I+\beta_{11}$ trial $_{\text {inverse }} \mathrm{BDI}+\beta_{12}$ trial $_{\text {linear }} *$ group $^{*} \mathrm{BDI}+\beta_{13}$ trial $_{\text {inverse }} *$ group $^{*} \mathrm{BDI} \mathrm{e}_{\mathrm{ti}}+\mathrm{u}_{0 \mathrm{i}}$

Where:

$\mathrm{t}=$ time point (1 to 20$)$,

$\mathrm{i}=$ subject

Trial $=20$ trial numbers, centred from $-9,5$ to +9.5 ,

Age $=$ centred continuous variable in years,

Gender $=$ dichotomous variable, $-1=\operatorname{man}, 1=$ woman,

Absolute pain threshold $=$ centred continuous variable in $\mathrm{mA}$,

Group $=$ dichotomous variable, $-1=$ pain-free controls, 1 = chronic pain sufferers,

$\mathrm{BDI}=$ centred continuous variable representing depressive complaints

$\mathrm{e}_{\mathrm{ti}}=$ error variance for subject $\mathrm{i}$ at time point $\mathrm{t}$. which is assumed to be the sum of an auto correlated component (AR1).

$\mathrm{u}_{0 \mathrm{i}}=$ personal deviation from the average intercept $\beta_{0}$

This model must be interpreted as follows:

$\beta_{0}=$ the outcome mean (Amplitude) at trial number 10 for a subject with a mean age and mean absolute pain threshold.

$\beta_{1}=$ the mean change over trials according to the linear part of the trend

$\beta_{2}=$ the inverse trial effect

$\beta_{3}=$ half the mean difference between men and women

$\beta_{4}=$ the mean change in amplitude per year

$\beta_{5}=$ the relation between the absolute pain threshold and the amplitude of the component

$\beta_{6}=$ Half the difference between pain-free controls and chronic pain sufferers

$B_{7}=$ The effect of group on the difference in amplitude between the adjacent trials, according to the linear part of the trend

$\beta_{8}=$ The effect of group on the difference in amplitude between the adjacent trials, according to the inverse part of the trend

$\beta_{9}=$ The effect of BDI on the difference in amplitude between the adjacent trials, according to the linear part of the trend

$\beta_{10}=$ The effect of BDI on the difference in amplitude between the adjacent trials, according to the inverse part of the trend

$\beta_{11}=$ The effect of BDI on the interaction between group and trial $\left.\right|_{\text {linear }}$

$\beta_{12}=$ The effect of $B D I$ on the interaction between group and trial $\left.\right|_{\text {inverse }}$ 


\section{References}

Arntz A, Lousberg R (1990) The effects of underestimated pain and their relationship to habituation. Behaviour Research and Therapy, 28: 15-28.

Beck AT, Steer RA, Garbin MG (1988) Psychometric properties of the Beck Depression Inventory: Twenty-five years of evaluation. Clinical Psychology Review, 8, 77-100.

Brands AM, Schmidt AJ (1987) Learning processes in the persistence behavior of chronic low back pain patients with repeated acute pain stimulation. Pain, 30, 329-337.

Bromm B, Meier W (1984) The intracutaneous stimulus: a new pain model for algesimetric studies. Methods and Findings in Experimental and Clinical Pharmacology, 6, 405-410.

Campbell CM, Edwards RR (2009) Mind-body interactions in pain: the neuophysiology of anxious and catastrophic pain-related thoughts. Trans/ational Research, 153, 97-101.

De Tommaso M, Libro G, Guido M, Losito L, Lamberti P, et al. (2005) Habituation of single CO2 laser evoked responses during interictal phase of migraine. Journal of Headache Pain, 6, 195-198.

Jasper HH (1958). The ten-twenty electrode system of the International Federation. Journal of Electroencephalography and Clinical Neurophysiology, 20, 371-375.

Nicassio PM, Wallston KA (1992) Longitudinal relationships among pain, sleep problems, and depression in rheumatoid arthritis. Journal of Abnormal Psychology, 101, 514-520.

Peters ML, Schmidt AJM, Van den Hout MA (1989) Chronic low back pain and the reaction to repeated acute pain stimulation. Pain, 39, 69-76.

Smith BW, Tooley EM, Montague EQ, Robinson AE, Cosper CJ, Mullins PG (2008) Habituation and sensitization to heat and cold pain in women with fibromyalgia and healthy controls. Pain, 140, 420-428.

Smith DBH, Michalewski HJ, Brent GA, Thompson LW (1980) Auditory averaged evoked potentials and aging: factors of stimulus and topography. Biological Psychology, 11, 135-151.

Tang NKY, Salkovskis PM, Hodges A, Wright KJ, Magdi H, Hester J (2008) Effects of mood on pain responses and pain tolerance: An experimental study in chronic back pain patients. Pain, 138, 392-401.

Tarkka IM, Treede RD (1993) Equivalent electrical source analysis of pain related somatosensory evoked potentials elicited by a CO2 laser. Journal of Clinical Neurophysiology, 10, 513-9.

Thompson RF, Spencer WA (1966) Habituation: A model phenomenon for the study of neuronal substrates of behavior. Psychological Review, 73, 16-43.

Valeriani M, Rambaud L, Mauguiére F (1996) Scalp topography and dipolar source modelling of potentials evoked by $\mathrm{CO} 2$ laser stimulation of the hand. Electroencephalography and Clinical Neurophysiology, 100, 343-53.

Valeriani M, de Tommaso M, Restuccia D, le Pera D, Giudo M, et al. (2005) Reduced habituation to experimental pain in migraine patients: a CO2 laser evoked potential study. Pain, 105, 57-64.

Van Breukelen GJP, Van Dijk KRA (2007) Use of covariates in randomized controlled trials. Journal of the International Neuropsychological Society, 13, 903-904. 
Woby SR, Roach NK, Urmston M, Watson PJ (2007) The relation between cognitive factors and levels of pain and disability in chronic low back pain patients presenting for physiotherapy. European Journal of Pain, 11, 869-877.

Young CC, Greenberg MA, Nicassio PM, Harpin E, Hubbard D (2008) Transition from acute to chronic pain and disability: a model including cognitive, affective and trauma factors. Pain, 134, 69-79. 



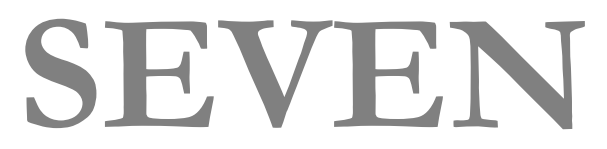

\title{
The Influence of COMT 158ValMet, BDNF Val66Met and OPRM1
}

\section{A118G polymorphisms on the cortical processing of experimental}

\author{
pain.
}

\author{
Reference \\ Vossen HGM, Kenis, GR, Rutten BP, Van Os J, Hermens H, Lousberg R (2009) The Influence of COMT \\ 158ValMet, BDNF Val66Met and OPRM1 A118G polymorphisms on the cortical processing of \\ experimental pain. Submitted for publication.
}




\section{Abstract}

Previous research has demonstrated that the COMT Val158Met, BDNF Val66Met and OPRM1 A118G polymorphisms impact on the experience of pain. These findings were extended using the phenotype of cortical processing of pain assessed with Event-Related Potentials. In the present study, a sample of 78 individuals with chronic low back pain complaints and 37 healthy controls underwent EEG registration. Event-Related Potentials were measured in response to electrical nociceptive stimuli and moderation by COMT Val158Met, BDNF Val66Met and OPRM1 A118G polymorphisms was assessed. The results demonstrated that these polymorphisms did not have a direct effect on the cortical pain processing. However, this effect is moderated by the presence of clinical pain complaints. When clinical pain complaints are present, the COMT Met allele and the BDNF Met allele seem to augment the cortical pain processing, while they reduce the pain processing in pain free controls. In the absence of chronic pain complaints the $\mathrm{G}$ allele of the OPRM1 reduces the pain processing, as hypothesized. However in the presence of chronic pain complaints the $G$ allele augments the pain processing. The current study gives evidence that the COMT Val158Met, BDNF Val66Met and OPRM1 A118G polymorphisms influence the cortical processing of experimental electrical pain stimuli, however the presence of chronic pain complaints alters the effect of these gene polymorphisms on the cortical processing of experimental noxious stimuli. 


\section{Introduction}

The experience of pain is subject to individual differences resulting from psychological factors (Carrillode-la-Peňa, 1992), behavioral factors (Fordyce et al, 1976; Craig, 1983) and biological factors (Melzack and Wall, 1965). There is rising interest in genetic factors, as these likely explain a substantial portion of the inter-individual differences in pain responses (Fillingim et al, 2008). Studies using genetically modified mice have proposed a large number of candidate 'pain genes' (LaCroix-Fralish, 2007). In human studies, however, the list to date is much shorter. In particular, three single nucleotide polymorphisms (SNPs) have been proposed to impact on pain perception; COMT Val158Met (rs4680), BDNF Val66Met (rs6265) and the OPRM1 A118G (rs1799971) (Šerý et al, 2005; Max et al, 2006; Buskila, 2007).

Catechol-O-Methyl Transferase (COMT) is an enzyme that catabolizes catecholamines and thus influences the dopaminergic and adrenergic/noradrenergic neurotransmission (Nackley et al. 2006). The COMT Val158Met polymorphism codes a of valine (val) by metionine (met) substitution at codon 158 , resulting in a decreased thermostability of the COMT protein. As such, the val158met polymorphism alters the in-vivo activity of the COMT enzyme. Val/Val homozygotes have higher levels of the COMT enzyme and correspondingly lower levels of D2 receptor neurotransmission leading to higher level of activation of the $\mu$-opioid system (George and Kertesz, 1987; Chen et al, 1993). On the other hand, met/met homozygotes have the lower levels of the COMT enzyme, resulting in increased dopaminergic neurotransmission. This genetic alteration in COMT activity influences the responsiveness of the $\mu$ opioid system, which is activated in response to pain and stressors, and typically reduces pain and stress responses (Zubieta et al, 2001; Ribeiro et al, 2005). Met/met homozygotes have decreased $\mu$-opioid system activation in response to pain (Zubieta et al, 2003). Therefore, Met/Met homozygotes are believed to be more sensitive to nociceptive stimuli than heterozygotes or Val/Val homozygotes.

Brain Derived Neurotrophic Factor (BDNF) is a neurotrophin that supports the growth, differentiation and survival of neurons in both peripheral and central nervous system. BDNF is released when nociceptors are activated and is involved in the activity-dependent pathogenesis of nociceptive pathways that may lead to chronification of pain. A genetic variation within the BDNF genes results in a valine to metionine substitution at codon 66 (val66met), leading to reduced secretion of the BDNF protein and impaired BDNF signalling. The BDNF Val66Met polymorphism may be implicated in depression (Sen et al, 2008) and is also hypothesized to influence pain mechanisms (Merighi et al, 2008). Despite the hypothesized influence of the Val66Met polymorphism on pain, an online search in PubMed and Medline with 'BDNF val66met' and 'pain' as keyword do not result in any publications. 
The A118G polymorphism of the $\mu$-Opioid Receptor 1 (OPRM1) gene replaces adenine with guanine (Fillingim et al. 2005), increasing the receptor affinity of $\beta$-endorphin three-fold (Bond et al. 1998). As such, it is hypothesized that this polymorphism increases the activity of the endogenous opioid system, which could be associated with a decreased response to nociceptive stimulation (Lötsch et al, 2006). The theory that this gene polymorphism influences pain mechanisms comes mainly from mice studies, which found that opioid receptor knock-out mice had increased nociceptive responsiveness (Sora et al,. 1997) In human studies, the OPRM1 $118 \mathrm{G}$ allele may increase the dose of morphine needed to achieve pain control (Klepstad et al, 2004; Chou et al. 2006). Furthermore, a study by Fillingim and co-workers (2005) reported that $\mathrm{G}$ allele carriers (infrequent allele) had significantly higher pressure pain thresholds than the A allele homozygotes. Lötsch and colleagues (2006) studied the influence of the G allele on the cortical pain processing to experimental pain stimuli. They concluded that ERP amplitudes (N1 component) of carriers of the $G$ allele were, on average, half as high as the amplitude of the noncarriers, suggesting a lower pain processing for the $G$ allele carriers.

The work described yield a suggestion that the three gene polymorphisms may influence pain responsiveness and that further work is required. It has been suggested that experimental designs represent a particularly powerful approach for the study of genetic effects on psychological phenotypes as they allow for controlled conditions and investigation of underlying mechanisms (van Os et al, 2008). In pain research, Event Related Potentials (ERPs) are frequently used as a more objective measure of pain, compared to subjective pain ratings, elicited by controlled exposure to noxious stimuli (Becker et al, 1993). Previous research has demonstrated positive correlations between the amplitude of specific peak components and the intensity of the pain stimulus (Garcia-Larrea et al, 1997; Kanda et al, 2002), as well as between ERP amplitudes and subjective pain ratings (Carmon et al, 1978; Chen et al, 1979).

Thus, the aim of this study was to investigate the influence of the genetic altered activity of the three gene polymorphisms described above on the cortical processing of experimental pain as measured with Event-Related Potentials. Results from previous studies (Valeriani et al, 2003; de Tommaso et al, 2008; de Tommaso et al, 2009) demonstrate that the pain-ERP to experimental noxious stimuli is influenced by the presence of chronic pain complaints. Therefore, any influence of genetic polymorphisms needs to be assessed in interaction with clinical pain. 
For every gene polymorphism three hypotheses were tested: (i) Carriers of the infrequent allele (Met allele for COMT and BDNF, and G allele for OPRM1) are expected to have different ${ }^{5}$ maximum peak amplitudes on the pain-specific ERP components (N1, P1, N2 and P2) (Bromm, 1984; Becker et al, 2000; Zaslansky et al, 1996; Kanda et al, 2002) in response to experimental pain stimuli compared to noncarriers. More specifically, concerning the $\mathrm{COMT} \mathrm{Val}^{158}$ Met polymorphism the Met allele carriers are expected to have larger ERP peak amplitudes compared to the non-carriers. For the BDNF Val ${ }^{66} \mathrm{Met}$ polymorphism, Met allele carriers are also expected to have larger amplitudes compared to the noncarriers. For the OPRM $1 \mathrm{~A}^{118} \mathrm{G}$, the $\mathrm{G}$ allele carriers are expected to have reduced amplitudes compared to non-carriers. (ii) Genetic effects are moderated by the experience of chronic pain. More specifically, we expected significant interaction effects between the polymorphisms and group (chronic low back pain patients versus pain free controls).

\section{Methods}

Approval has been obtained from the medical ethics committee of the Academic Hospital Maastricht, on January, 6th, 2005. All subjects gave their verbal and written informed consent prior to the experiment.

\section{Subjects}

Two groups of subjects were included in this study. The first group consisted of seventy-eight subjects with low back pain complaints. These subjects were drawn from the general population (via advertisements, distributed door to door) and were required to have low back pain for at least six months with no other interfering pain complaints. Exclusion criteria were the use of psychoactive drugs in general and the use of analgesics more than eight hours before the experiment. The second group consisted of thirty-seven pain-free subjects. The age ranged from 18 to 65 years. Exclusion criteria were the use of psychoactive drugs in general and the use of analgesics more than eight hours before the experiment. Participation was rewarded with €25,--

\footnotetext{
${ }^{5}$ Concerning the COMT Val158Met polymorphism the Met allele carriers are expected to have stronger ERP peak amplitudes compared to the non-carriers. For the BDNF Val66Met polymorphism the Met allele carriers are also expected to have stronger amplitudes compared to the non-carriers. For the OPRM1 A118G the G allele carriers are expected to have less strong amplitudes compared to the non-carriers.
} 


\section{Stimuli}

The stimuli used in this study were electrical pulses of ten milliseconds duration. Stimuli were administered intracutaneuously on the left middle finger. For each participant, five different intensities based on that participant's sensation and pain thresholds were administered. Of the five intensities, one was the pain threshold and the other four were defined relative to this pain threshold, namely $-50 \%$, $25 \%,+25 \%$ and $+50 \%$ of the threshold range which was defined as the range between the sensation threshold and the pain threshold. The sensation threshold was determined by first administering stimuli at zero intensity and then gradually increasing the intensity until the stimuli were experienced consciously. Once experienced consciously (sensation threshold), stimuli were once again administered with an intensity that gradually increased from the sensation threshold until the stimuli were defined as painful by the participant (pain threshold). This procedure was repeated three times in order to generate a reliable measurement.

\section{Rating paradigm}

The stimuli were presented using a rating paradigm (Bromm, \& Meier, 1984). The paradigm consisted of 112150 stimuli. The five intensities mentioned above were presented semi-randomly. The inter-stimulus interval (ISI) ranged from 9 to 11 seconds. Subjects were asked to rate the intensity of each stimulus on a Numeric Rating Scale (NRS) from 0 to 100 , whereby 0 is no sensation and 100 is the most excruciating pain imaginable. The first stimulus of the paradigm was set at the pain threshold. As a reference, subjects were instructed that this intensity was ' 60 '.

\section{EEG recording}

All EEG recordings were conducted in an electrically- and sound-shielded cubicle $\left(3 * 4 \mathrm{~m}^{2}\right) . \mathrm{Ag} / \mathrm{AgCl}$ electrodes were placed on $\mathrm{Fz}, \mathrm{Cz}, \mathrm{Pz}, \mathrm{C} 3, \mathrm{C} 4, \mathrm{~T} 3$ and T4 using the international 10-20 system (Jaspers, 1958). Impedances were kept below $5 \mathrm{k} \Omega$. A reference electrode was placed on each ear lobe. To control for possible vertical eye movements, an electro-oculogram (EOG) electrode was placed 1 centimetre under the midline of the right eye. A ground electrode was placed at Fpz. All electrodes were fixed using 10-20 conductive paste. Neuroscan 4.3 software was used for EEG recording. 


\section{Procedure}

Before starting the experiment, subjects were informed about the purpose of the study. Subjects were told that they would undergo EEG-registration while they received electric shocks. After signing the informed consent form, EEG electrodes were placed and the shock electrode was attached to the top of the left middle finger as described by Bromm and Meier (1984): a small opening in the upper layer of the skin was prepared using a dental gimlet. Care was taken that this procedure was not painful. In the prepared opening, a platinum electrode was placed and fixed with tape. Next, the sensation and pain threshold were determined and after that, the Rating paradigm was initiated.

\section{Genetic analyses}

Buccal cell samples were collected with sterile swabs (Omniswab, Whatman ${ }^{\circledR}$ ). DNA was extracted using QIAamp DNA Mini Kits (Qiagen). In total, seven SNPs within the COMT gene were determined. The following three SNPs were genotyped using TaqMan ${ }^{\circledR}$ SNP Genotyping assays (Applied Biosystems): rs4680 (assay ID C_25746809_50), rs6265 (assay ID C_11592758_10), and rs1799971 (assay ID C__8950074_1_). All assays were run on a 7900HT Fast Real-Time PCR System (Applied Biosystems).

\section{ERPs}

EEG was recorded with $1000 \mathrm{~Hz}$ sampling rate, using Neuroscan 4.3 software. Trials were selected from the continuous EEG, from $200 \mathrm{~ms}$ prior to the stimulus until $1500 \mathrm{~ms}$ post-stimulus. Data was offline filtered (bandpass $0-50 \mathrm{~Hz}$ ) and baseline corrected. Trials with EOG activity exceeding $+75 \mathrm{~mA}$ and $-75 \mathrm{~mA}$ were excluded from the analyses.

\section{Statistical analyses}

Multilevel random regression analyses were performed because of the hierarchical structure of the ERP data. Trials (level 1) were clustered in individuals (level 2). The following maximum peak components, N1 (20-55msec), P1 (56-95msec), N2 (96-145msec), P2 (146-300msec), served as dependent variable. These components have been shown to be related to the processing of stimulus intensity (Kanda et al, 2002; Becker et al, 2000; Zaslansky et al, 1996; Bromm, 1984). The N1 and P1 components are considered to be involved with the sensory processing and generated by activity in the primary and secondary somatosensory cortices. The N2 and P2 components are thought to be generated by neurons 
in the cingulate cortex, which is part of the limbic system which in turn is responsible for the emotional processing of pain stimuli (Tarkka \& Treede, 1993). Intensity, trial number, sensation threshold, pain threshold, age, gender, group and intensity of the previous stimulus served as independent variables in a basic model. Trial number was divided in a linear effect, a quadratic and an inverse effect ${ }^{6}$. The

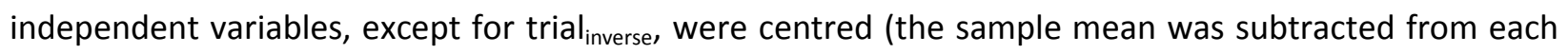
individual score) (Van Breukelen \& Van Dijk, 2007).

In order to study the influences of the three gene polymorphisms they were first added (separately) as main factors to the basic model. The polymorphisms were included in the analyses as dichotomous variables (allele carriers and non-carriers). In order to test the second hypothesis, interactions between group and the gene polymorphisms were added.

The Scaled Identity covariance structure was used in the multilevel analyses. A random intercept was included. All statistical analyses were performed using SPSS 16.0. All p-values $\leq 0.007$ (two-tailed with Bonferroni correction for the number of cranial locations, namely seven) were considered as statistically significant. Effects were considered marginally significant when p-values were between 0.050 and 0.008 .

\section{Results}

Demographic and allele/genotype distribution are displayed in table 1. The pain free control group consisted of 21 COMT Met carriers and 15 non-carriers, 11 BDNF Met carriers and 26 non-carriers, and 8 OPMR1 G carriers and 29 non-carriers. The chronic pain group consisted of 55 COMT Met carriers and 22 non-carriers, 24 BDNF Met carriers and 54 non-carriers, and 15 OPRM1 G carriers and 61 noncarriers. Crosstabs revealed no significant difference in distribution between the healthy control group and chronic pain group (resp. $p=0.167, p=0.910, p=0.815$ ).

\section{The COMT Val158Met gene polymorphism}

The first hypothesis tested for a difference in cortical pain processing between the COMT met allele carriers and the non-carriers. The results show that the COMT met allele does not directly explain variance of the pain-ERP and therefore do not support the first hypothesis.

The second hypothesis concerned a moderation of the effect of the Met allele by chronic pain complaints. A marginally significant interaction between group (chronic pain patients vs. pain free controls) and the Met allele was found on the N2-component of $\mathrm{T} 4(\mathrm{~B}=-2.49, \mathrm{SE}=0.79, \mathrm{p}=0.002)$. 
Post-hoc analyses per intensity revealed that this interaction was only significant for the intensities equal and above the pain threshold. Figure 1 illustrates the interaction effect and shows that in the pain free control group the COMT met carriers have a lower maximum peak amplitude (less negative) than the non-carriers. In the chronic pain group the carriers have higher maximum peak amplitudes (more negative) compared to the non-carriers.

\section{The BDNF Val66Met gene polymorphism}

The results for the first hypothesis did not show any direct effect of the BDNF 66Met allele on the painERP, indicating that there is no difference in cortical pain processing between Met allele carriers and non-carriers. Hereby, the first hypothesis is not supported.

Analyses, investigating the moderating effect of chronic pain complaints on the effect of the BDNF Met allele, revealed a significant interaction between group (chronic pain group and pain free control group) and the BDNF Met allele on the P1- component of $C z(B=1.30, S E=0.41, p=0.002)$ and a marginally significant interaction on the $P 1$-component of $C 3(B=1.118, S E=0.442, p=0.013)$ and $C 4(B=1.34, S E=$ $0.50, p=0.009)$. Post-hoc analyses per intensity demonstrated that these interactions were significant for the intensities above the pain threshold and not for the intensities below the pain threshold. Figure 2 shows the interaction effect on $\mathrm{Cz}$. Similar to the results of COMT, in the pain free control group the COMT met carriers have a lower maximum peak amplitude (less positive) than the non-carriers. In the chronic pain group the carriers have higher maximum peak amplitudes (more positive) than the noncarriers.

\section{The OPRM1 A118G gene polymorphism}

Similar to COMT Val158Met and BDNF Val66Met, no main effect of the OPRM1 A118G polymorphism on the pain-ERP was found. Carriers of the $G$ allele did not respond differently to experimental nociceptive stimuli from non-carriers.

The results of the second hypothesis revealed only a marginally significant interaction between group and OPRM1 G allele on the N2-component of $F z(B=-1.66, S E=0.66, p=0.014)$. Post-hoc analyses for the different intensities demonstrated that this intensity was actually only significant for the lowest intensity ( $50 \%$ below the pain threshold). This interaction indicates that in the pain free group the maximum peak amplitude of the non-carriers is higher (more negative) compared to the carriers. In the 
chronic pain group the maximum peak amplitude of the carriers is higher (more negative) compared to the non-carriers (figure 3).

Based on the knowledge that both the COMT Val158Met and the OPRM1 A118G polymorphisms act on the mu-opioid system, post-hoc analyses were performed to test whether these two polymorphisms influenced each other. The results showed no confounding influences or interactions concerning these two polymorphisms. Furthermore, both COMT Val ${ }^{158}$ Met and BDNF Val ${ }^{66}$ Met polymorphisms have been shown to be involved in the development of depressive disorders. Moreover, there is a well known interplay between pain and depression. Post-hoc analyses revealed that individuals with chronic pain complaints reported more depressive symptoms (measured with the Beck Depression Inventory) compared to the pain free controls. However, depressive symptoms did not seem to have any influence on the interactions between group and genotype.

\section{Discussion}

The main goal of this study was to investigate the influence of three 'pain candidate gene polymorphisms' (COMT Val158Met, BDNF Val66Met and OPRM1 A118G), on pain Event Related Potentials. It was investigated whether these polymorphisms influenced the cortical processing of experimental pain and whether the presence of clinical pain influences this.

The results demonstrate that the COMT Val158Met polymorphism did not have a direct (main) effect on the pain-ERP. In other words, independent of the covariates (intensity, trial number, sensation threshold group etc.), there was no difference in cortical pain processing between Met allele carriers and non-carriers. There was, however, an interaction between group and the Met allele (N2-component of T4), indicating that the effect of the COMT Met allele on the pain-ERP was moderated by the presence of chronic pain complaints. When chronic pain complaints are present, COMT Met carriers cortically process pain stimuli stronger than the non-carriers. When chronic pain complaints are absent the carriers have a weaker pain processing compared to the non-carriers. Thus, in this study, the expected pain augmenting effect of the COMT Met allele only occurs in a group of chronic pain patients. In a pain free population the Met allele has rather a beneficiary effect on the cortical processing of pain. Concerning the BDNF Val66Met polymorphism, the results show no differences in cortical pain processing between Met carriers and non-carriers. There was, however, a significant interaction between group and the Met allele at several locations. Similar to the results of the COMT polymorphism, the interaction between group and the BDNF Met allele demonstrates that when chronic low back pain 
complaints are present, the Met allele carriers have stronger ERP amplitudes (P1 component) compared to the non-carriers. When chronic pain complaints are not present the non-carriers display stronger amplitudes. Again the expected pain augmenting effect of the BDNF Met allele only occurs when chronic pain complaints are present.

The mu-opioid polymorphism OPRM1 A118G is the only one of these three polymorphisms that has previously been studied with the use of Event-Related Potentials. Lötsch and colleagues (2006) found that the $G$ allele carriers had significantly lower peak amplitude compared to the non-carriers. In the present study such a significant effect of the $G$ allele was not found. This difference in result may be explained by differences in pain stimulus (nasal $\mathrm{CO}_{2}$ stimulation vs. electrical stimulation), stimulus duration (200ms vs. $10 \mathrm{~ms}$ ) and paradigm ( 2 intensities vs. 5 intensities). There was, however only a marginally significant, a interaction between the $\mathrm{G}$ allele and group. This interaction indicated that when chronic pain complaints were not present the effect of the $G$ allele was as hypothesized, $G$ allele carriers had reduced pain processing as compared to the non-carriers. However, when pain complaints were present, the $\mathrm{G}$ allele carriers had a stronger cortical pain processing than the non-carriers. This means that the presence of chronic pain complaints changes the effect of the $G$ allele. Where normally the $G$ allele is related to a reduced response to pain, the presence of clinical pain complaints turns this effect around.

Thus the results demonstrate that the presence of chronic pain complaints moderates the influence of the COMT, BDNF and OPRM1 polymorphisms on the cortical processing of experimental pain stimuli. There are several possible explanations for this effect. First, an explanation concerning the COMT $\mathrm{Val}^{158}$ Met polymorphism might be alterations in endogeneous opioid system that are associated with chronic pain complaints (Bruehl, 1999). Jensen and colleagues (2009) reported that COMT Val ${ }^{158}$ Met differences may be more expressed in individuals where the inhibitory nociceptive system is already challenged and sensitive. Furthermore, it is tempting to speculate that epigenetic mechanisms play a role in this. Chronic exposures to environmental factors may result in dynamic changes of neuronal gene expression which may persist over time but can also be reversed (Jaenisch \& Bird, 2003. Future research is needed to test this speculation.

The main limitation of this study is most likely the relatively small number of healthy controls. Therefore only the alleles and not the genotypes could be studied. Studying genotypes could have given information about possible differences in cortical processing of pain stimuli, between the heterozygotes and homozygotes of the alleles of interest. . For that reason, further research with larger populations is needed for increased power. In addition stratified samples on genotype would result in an equal 
distribution of genotype. Finally, including neuroimaging tools in further research would give more detailed information on which brain areas are influenced by these polymorphisms, since Event-Related Potentials have poor spatial resolution.

The current study gives evidence that the COMT Val158Met, BDNF Val66Met and OPRM1 A118G polymorphisms influence the cortical processing of experimental electrical pain stimuli, however not in a direct manner but rather under moderation of the presence of chronic pain complaints. 


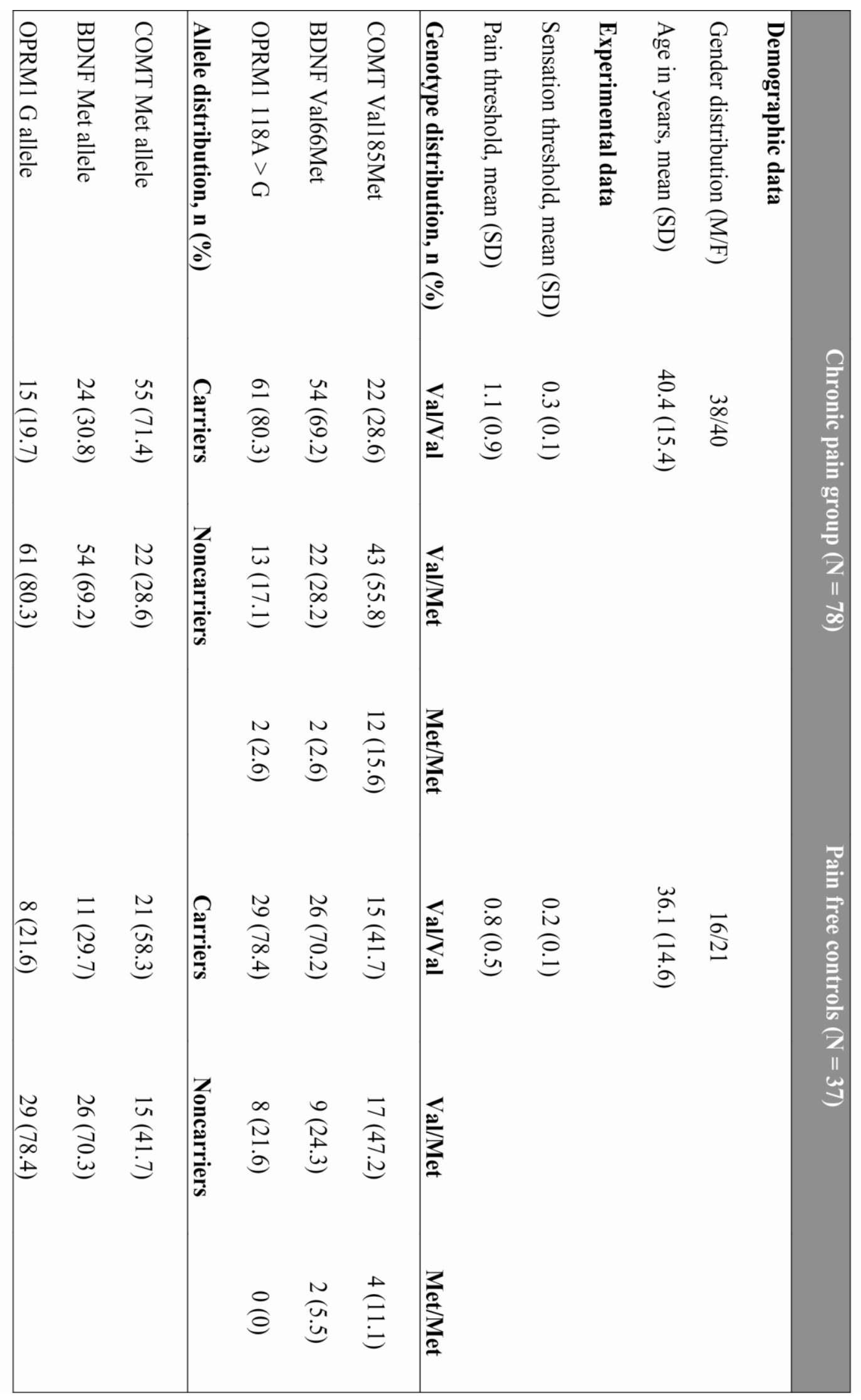

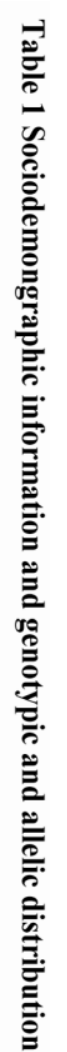




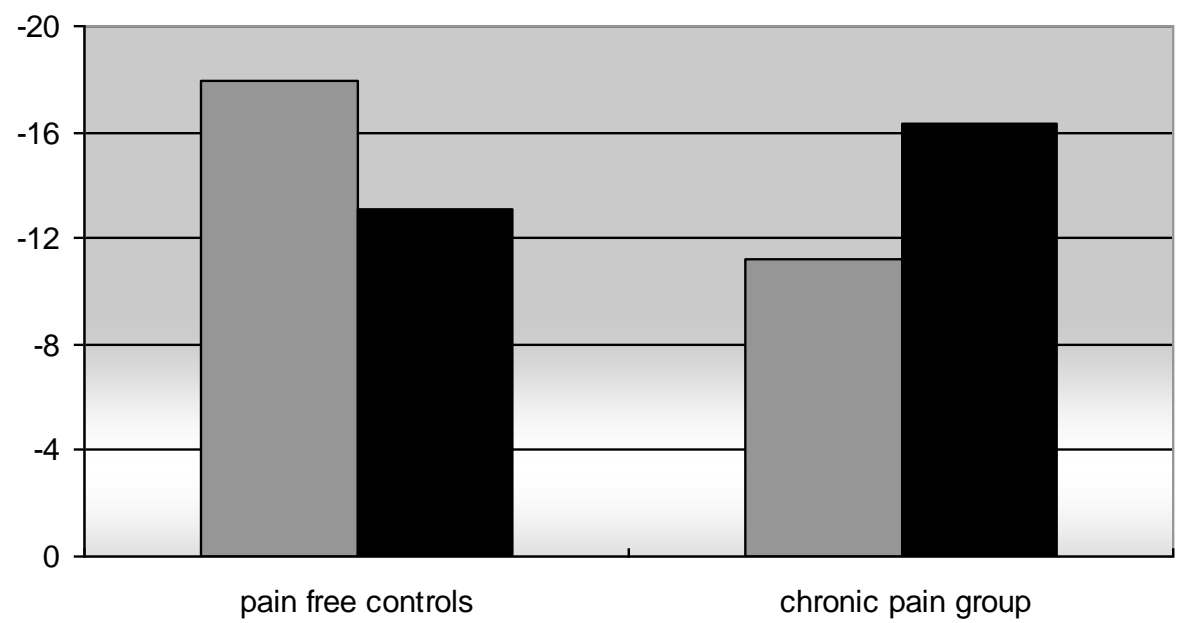

Figure 1. Interaction between the COMT 158Met allele and group. Maximum peak amplitudes of the N2-component at C4. Note that the $y$-axis displays negative numbers since this concerns a negative ERP component

$\square$ BDNF 66Met non-carriers

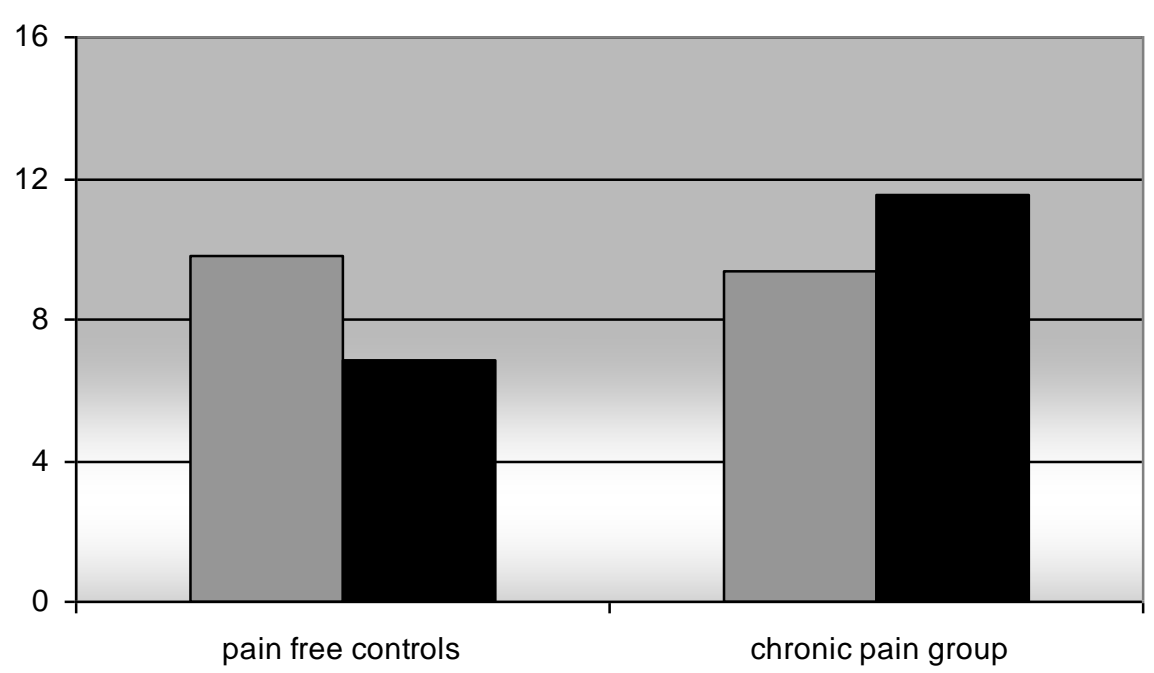

Figure 2. Interaction between the BDNF 66Met allele and group. Maximum peak amplitudes of the P1component at $\mathrm{Cz}$. 
口 OPRM1 118G non-carriers

OPRM1 118G carriers

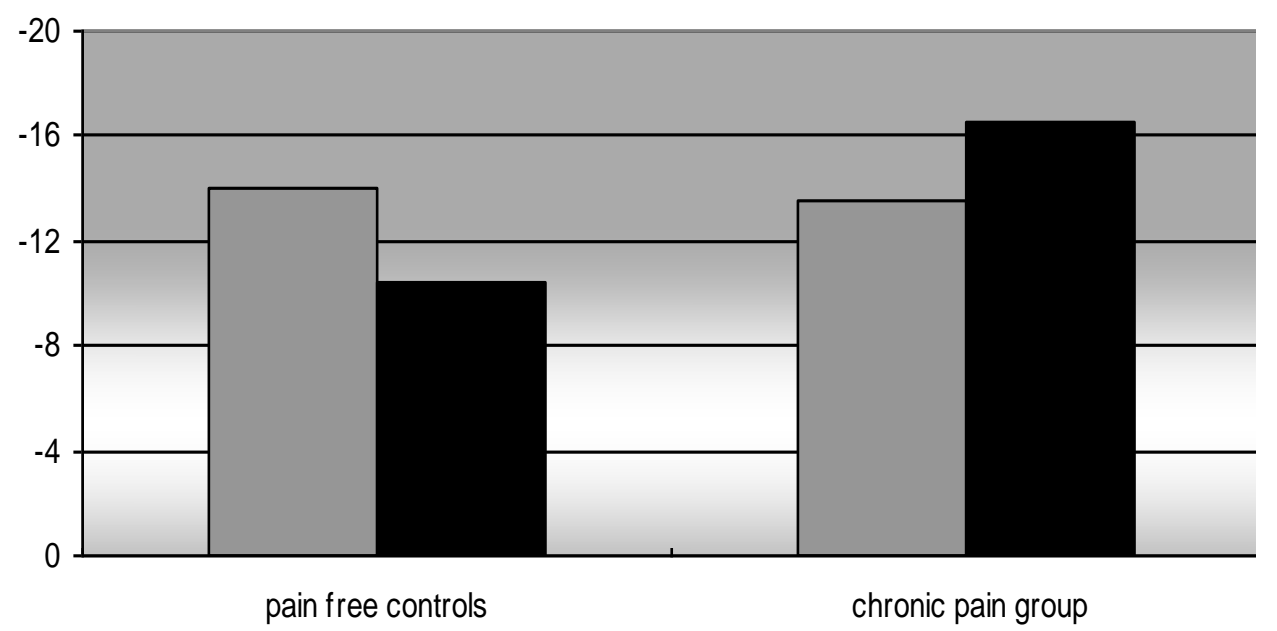

Figure 3. Interaction between the OPRM1 118G allele and group. Maximum peak amplitudes of the N2-component at Fz. Note that the $y$-axis displays negative numbers since this concerns a negative ERP component. 


\section{References}

Becker DE, Yingling CD, Fein, G (1993) Identification of pain, intensity and P300 components in the pain evoked potential. Electroencephalography and Clinical Neurophysiology, 88, 209-301.

Becker DE, Haley DW, Urena VM, Yingling CD (2000) Pain measurement with evoked potentials: combination of subjective ratings, randomized intensities, and long interstimulus intervals procedures a P300-like confound. Pain, 84, 37-47.

Bond C, LaForge KS, Tian M, Melia D, Zhang S, Borg L, Gong J, Schluger J, Strong JA, Leal SM, Tischfield JA, Kreek MJ, Yu L (1998) Single-Nucleotide Polymorphism in the human $\mu$-opioid receptor gene alters $\beta$-endorphin binding and activity: Possible implications for opiate addiction. Proceedings of National Academy of Science USA, 95, 96089613.

Bromm B, \& Meier W (1984) The intracutaneous stimulus: a new pain model for algesimetric studies. Methods \& Findings in Experimental Clinical Pharmacology, 6, 405-410.

Bromm B (1984) Pain-related components in the cerebral potential: experimental and multivariate statistical approaches. In Pain measurement in man: Neurophysiological correlates of pain (Ed. Bromm B), pp. 257-290. Elsevier: Amsterdam.

Buskila D (2007) Genetics of chronic pain states. Best Practice \& Research Clinical Rheumatology, 21, 535-547.

Carmon A, Dotan Y, Sarne Y (1978) Correlation of Subjective Pain Experience with Cerebral Evoked Responses to Noxious Thermal Stimulations. Experimental Brain Research, 33, 445-453.

Carrillo-de-la-Peňa MT (1992) ERP augmenting/reducing and sensation seeking: a critical review. International Journal of Psychophysiology, 12, 211-220.

Chen ACN, Chapman CR, Harkins SW (1979) Brain potentials are functional correlates of induced pain in man. Pain, 6, 365-374.

Chen JF, Aloyo VF, Weiss B (1993) Continuous treatment with the D2-dopamine receptor agonist decreases D2 dopamine receptors, D2 dopamine receptor messenger RNA and pro-enkephalin messenger RNA, and increases mu opioid receptors in mouse striatum. Neuroscience, 54, 669-80.

Chou WY, Yang LC, Lu HF, Ko JY, Wang CH, Lin SH, Lee TH, Concejero A, Hsu CJ (2006) Association of mu-opioid receptor gene polymorphism (A118G) with variations in morphine consumption for analgesia after total knee arthroplasty. Acta Anaesthesiologica Scandinavia, 50, 787-792.

Coull JA, Beggs S, Boudreau D, Boivin D, Tsuda M, Inoue K, Gravel C, Salter W, De Koninck Y (2005) BDNF from microglia causes the shift in neuronal anion gradient underlying neuropathic pain. Nature, 438, 1017-1021.

Craig KD (1983) Modelling and social learning factors in chronic pain. Advances in Pain Research and Therapy, 5, 813-827.

De Tommaso M, Libro G, Guido M, Losito L, Lamberti P, Livrea P (2005) Habituation of single CO2 laser evoked responses during interictal phase of migraine. Journal of Headache Pain, 6, 195-198

De Tommaso M, Libro G, Difruscolo O, Sardaro M, Serpino C, Calabrese R, Vecchio E, Livrea P (2009) Laser evoked potentials in carpal tunnel syndrome. Clinical Neurophysiology, 120, 353-359. 
Diatckenko L, Slade GD, Nackley AG, Bhalang K, Sigurdsson A, Belfer I, Goldman D, Xu K, Shabalina SA, Shangin D, Max MB, Makarov SS, Maixer W (2005) Genetic basis for individual variations in pain perception and the development of a chronic pain condition. Human Molecular Genetics, 14, 135-143.

Fillingim RB, Kaplan L, Staud R, Ness TJ, Glover TL, Campbell CM, Mogil JS, Wallace MR (2005) The A118G single nucleotide polymorphism of the mu-opioid receptor gene (OPRM1) is associated with pressure pain sensitivity in humans. Journal of Pain, 6, 159-167.

Fillingim RB, Wallace MR, Herbstman DM, Ribeiro-Dasilva M, Staud R (2008) Genetic contributions to pain: a review of findings in humans. Oral Diseases, 14, 673-682.

Fordyce WE, Fowler RS, Lehmann JF, DeLateur BJ, Sand PL, Trieschmann RB (1973) Operant conditioning in the treatment of chronic pain. Archives of Physical Medicine and Rehabilitation, 54, 339-408.

Garcia-Larrea L, Peyron R, Laurent B, Mauguiere F (1997) Association and dissociation between laser-evoke potentials and pain perception. Neuroreport, 8, 3785-3789.

George SR, Kertesz M (1987) Met-enkephalin concentrations in striatum respond reciprocally to alterations in dopamine neurotransmission. Peptides, 8, 487-92

Jasper HH (1958) The ten-twenty electrode system of the International Federation. Journal of Electroencephalography and Clinical Neurophysiology, 20, 371-375.

Kanda M, Matsuhashi M, Sawamoto N, Oga T, Mima T, Nagamine T, Shibasaki H (2002) Cortical potentials related to assessment of pain intensity with visual analogue scale (VAS). Clinical Neurophysiology, 113, 1013-1024.

Klepstad P, Rakvåk TT, Kaasa S, Holthe M, Dale O, Borchgrevink PC, Baar C, Vikan T, Krokan HE, Skorpen F (2204) The $118 \mathrm{~A}>\mathrm{G}$ polymorphism in the human $\mu$-opioid receptor gene may increase morphine requirements in patients with pain caused by malignant disease. Acta Aneasthesiologica Scandinavia, 48, 1232-1239.

LaCroix-Fralish ML, Ledoux JB, Mogil JS (2007) The Pain Gene Database: An interactive web browser of pain-related transgenetic knockout studies. Pain, 131, 3.e1-3.e4.

Lötsch J, Stuck B, Hummel T (2006) The Human $\mu$-Opioid Receptor Gene Polymorphism 118A > G Decreases Cortical Activation in Response to Specific Nociceptive Stimulation. Behavioural Neuroscience, 120, 1218-1224.

Max MB, Wu T, Atlas SJ, Edwards RR, Haythornthwaite JA, Bollettino AF, Hipp HS, Mcknight CD, Osman IA, Crawford EN, Pao M, Nejim J, Kingman A, Aisen DC, Scully MA, Keller RB, Goldman D, Belfer I (2006) A clinical genetic method to identify mechanisms by which pain causes depression and anxiety. Molecular Pain, 2, 14-27.

Melzack R, Wall PD (1965) Pain mechanisms: a new theory. Science, 150, 971-979.

Merighi A, Salio C, Ghirri A, Lossi L Ferrini F, Betelli C, Bardoni R (2008) BDNF as a pain modulator. Progress in neurobiology, 85, 297-317.

Nackley AG, Shabalina SA, Tchivileva IE, Satterfield K, Korchynskyi O, Makarov SS, Maixner W, Diatchenko L (2006) Human Catechol-O-Methyltransferase Haplotypes Modulate Protein Expression by Altering mRNA Secondary Structure. Science, 314, 1930-1933.

Os van J, Rutten BP, Poulton R (2008) Gene-environment interactions in schizophrenia: Review of epidemiological findings and future directions. Schizophrenic Bulletin, 34, 1066-82. 
Pezet S, McMahon SB (2006) Neurotrophins: mediators and modulators of pain. Annual Review of Neuroscience, 29, 507-538.

Sen S, Duman R, Sanacora G (2008) Serum brain-derived neurotrophic factor, depression and antidepressant medications: meta-analyses and implications. Biological Psychiatry, 15, 527-532.

Šerý O, Hrazdilová O, Matalová E, Ševčik P (2005) Pain research update from a genetic point of view. Pain Practice, 5, 341-348.

Tarkka IM, Treede RD (1993) Equivalent electrical source analysis of pain related somatosensory evoked potentials elicited by a CO2 laser. Journal of Clinical Neurophysiology, 10, 513-519.

Valeriani M, de Tommaso M, Restuccia D, le Pera D, Giudo M, lannetti GD, Libro G, Truini A, Di Trapani G, Puca F, Tonali P, Cruccu G (2005) Reduced habituation to experimental pain in migraine patients: a CO2 laser evoked potential study. Pain, 105, 57-64.

Van Breukelen GJP, Van Dijk KRA (2007) Use of covariates in randomized controlled trials. Journal of International Neuropsychology Society, 13, 903-904.

Zaslansky R, Sprecher E, Tenke CE, Hemli JA, Yarnitsky G (1996) The P300 in evoked potentials. Pain, 66, 39-49.

Zubieta JK, Smith YR, Bueller JA, Xu K, Kilbourn MR, Jewett DM, Meyer CR, Koeppe RA, Stohler CS (2001) Regional mu-opioid receptor regulation of sensory and affective Dimensions of Pain. Science, 293, 311-315

Zubieta JK, Heitzeg MM, Smith YR, Bueller JA, Xu K, Xu Y, Koeppe RA, Stohler CS, Goldman D (2003). COMT val $^{158}$ met Genotype Affects $\mu$-Opioid neurotransmitter Responses to a Pain Stressor. Science, 299, 1240-1243. 


EIGHT

General Discussion 
Pain has been studied and is still studied extensively because it is a complex phenomenon and because a number of unanswered questions regarding pain still remain. Despite a considerable progress in the development of (chronic) pain treatments, the occurrence of pain that lacks evident underlying pathology is still poorly understood and treatment often yields non-optimal results (Mes, 2007). The primary aim of this dissertation was to obtain more fundamental knowledge about Event (Pain) Related Potentials (ERPs). ERPs represent the cortical activity that occurs in response to painful stimuli and, as such, measure the involved, multidimensional, processes involved in pain.

Chapter 2 of this dissertation described a study in which the multidimensional character of pain was demonstrated using the ERP technique. This study showed that the personality trait neuroticism influences the cortical processing of pain in healthy subjects. More specifically, the subfacets anxiety and depression were found to exhibit opposite directions of effect on the ERP, despite their strong positive correlation with one another on the scale/questionnaire level. Further, in this study, an alternative technique for analyzing ERPs was employed. The rationale for this alternative technique was that the ERP amplitude at any given point in time (latency) is considered to be a function of measurement/technical variables, paradigm variables and variables related to neurophysiological, (neuro)psychological and cultural characteristics within the subject. Therefore, restricting analyses to merely maximum peak components was thought to limit the amount of information that can be obtained from the pain-ERP. Performing linear regressions for every 10 milliseconds of the entire ERP latency, revealed specific latency ranges, not only surrounding peak components, where personality factors were significant predictors of ERP amplitude. Although this provided insight regarding how we can best explain the variance around an ERP curve, the translation of these results to the actual pain experience was problematic because these effects were not found in pain ratings. It was also recognized that the statistical method of conducting multiple linear regressions (150) substantially increases the risk of type I errors. Furthermore, because the study sample comprised only healthy university students, the question of whether similar results can be replicated in chronic pain patients arose.

The above questions and concerns led to the initiation of a second experimental study in which chronic pain patients as well as pain-free control subjects were tested. This second study had three specific aims. First, the study endeavoured to investigate whether the multilevel analysis technique is an appropriate technique for the statistical handling of ERP data. Second, the study sought to explore how ERPs relate to subjective ratings in the laboratory and in daily life. Third, the study investigated differences in cortical processing between chronic pain patients and pain-free control subjects. 


\section{Multilevel analysis as a new and crucial technique in ERP analyses}

The ERP is a rather complex measure. Unlike, for instance, a VAS measure, the ERP does not provide only one measure but rather multiple measures, i.e. amplitudes, latencies, maximum peak amplitudes and areas. Indeed ERPs can offer more information than unidimensional measures such as VAS or pain ratings. However, the analysis and use of ERP data is very complex. ERP trials are therefore often averaged (aggregation). This not only makes it easier to handle the data, it also improves the signal to noise ratio (Fabiani et al, 2000). The author of this thesis, however, contends that the aggregation of data limits the information that can be obtained via the ERP technique. Particularly in pain research, it seems logical that people would react differently to the first stimulus they are presented with than they would to e.g. the thirtieth stimulus. This phenomenon, called habituation, refers to a decrease in response to a stimulus that is presented repeatedly (Cohen et al, 1997). When averaged ERPs are used in statistical tests, such as t-tests and analyses of variance (ANOVAs), trial-to-trial variability cannot be tested or corrected for. From a theoretical perspective, the multilevel regression technique may be a more appropriate technique for the analysis of ERPs and habituation within ERPs because single trial data can be analyzed and aggregation is unnecessary. Subsequently, within-subject variance and covariance can be modelled and studied in detail. Furthermore, since in multilevel analyses cases with missing values are not list-wise deleted the sample stays intact. All these theoretical advantages were tested the study described in chapter 3 . The study confirmed the hypothesized advantages of the multilevel regression technique. The use of single trial data and the possibility to model beyond polynomial contrasts led to the finding that the amplitude of specific pain components of the ERP tend to follow not only a linear but also an inverse $(1 / x)$ decrease pattern across trials (habituation). Furthermore, because it was possible to test for random slopes (within-subject variance), it was established that individuals differ significantly in their reactions across stimulus intensities and in their habituation patterns, which, intuitively, makes sense. Finally, the inclusion of single trial data made it possible to test within-subject covariates and this led to the establishment of an interaction between the intensity of the previous stimulus and the intensity of the present stimulus. In other words, how people react cortically to certain stimulus intensities depends on the intensity of the prior stimulus. Again, intuitively, this appears to makes sense, even though this has not previously been mentioned in the literature.

Evidently, the results of the this study clearly suggest that the multilevel technique - a technique that allows to investigate the variance around ERP amplitudes in more detail than more common statistical 
methods do - is a promising, and in the authors 'opinion even necessary, statistical technique that may enable us to further unravel the pain-ERP.

\section{The pain-ERP in relation to subjective ratings of experimental pain and clinical pain}

ERPs are often used to draw conclusions about the subjective experience of pain. This, however, is rather complex. Previous studies have demonstrated correlations between specific pain-ERP peak components and subjective pain ratings (Harkins \& Chapman, 1978; Chen et al, 1979; Miltner et al, 1987; Garcia-Larrea et al, 1997). Although these bivariate correlations suggest a direct link between specific peak components and the subjective experience of pain, such correlations cannot control for confounding factors. The results presented in this dissertation (chapter 4) demonstrated that the relation between the pain-ERP and the subjective experience of pain is moderated by habituation over repeated trials and by the intensity of the pain stimulus. More specifically, the relation between specific pain-ERP components (especially P1 and P3) was found to diminish as the number of repeated trials increased. This implies that the translation of ERP amplitudes to subjective experience is dependent on the number of repeated trials. Furthermore, the relation between the pain-ERP and subjective pain ratings was found to increase as the intensity of the stimulus increased. Perhaps these interactions could be attributed to factors such as bias and catastrophizing. In fact, a major difference between the ERP and subjective ratings is that subjective ratings are influenced by various kinds of bias, such as individual differences in the ability to accurately describe or discriminate pain stimuli as well as numeric preference, while the amplitudes of the pain-ERP are not (Carlsson, 1983; Herr et al, 2004). The interaction effect concerning stimulus intensity found in the this study whereby the relation between ERP and subjective ratings increased as stimulus intensity increased may actually be because, as stimulus intensity increases, bias play less of a role. Furthermore, the finding that there is interaction concerning the effect of trial number may indicate that, as individuals habituate to a stimulus, factors such as bias may play an increasing role. This may explain why the relation between the pain-ERP and subjective ratings weakened. Naturally, this potential explanation needs to be validated in future research.

Aside from the relation between the pain-ERP and subjective ratings, measured in the lab, an important issue is the transferability of experimental findings to daily life. Does the pain-ERP have predictive value for chronic pain complaints in daily life? The results presented in chapter 5 demonstrate this is indeed the case. The N2 components at $\mathrm{Cz}$ and $\mathrm{C} 4$ were significant predictors of the amount of pain 
experienced in daily life. These specific ERP components were even stronger predictors compared to subjective ratings of experimental pain. These results suggest that based on the way people cortically react to experimental pain stimuli we can predict how much pain they experience in daily life. This is interesting, since this indicates that the ERP might be helpful in diagnostics. This point is discussed more extensively below.

Clearly, the above suggests that the pain-ERP is not proportionally related to the subjective experience of pain. Stimulus properties such as intensity and processes such as habituation need to be taken into account when interpreting changes in the specific pain components of the ERP.

\section{Cortical differences between chronic pain patients and pain-free control subjects}

One of the most important questions in pain research is why some people develop chronic pain and others do not. What factors cause an acute pain complaint to become chronic? In this dissertation, two factors that may distinguish the cortical processing of chronic pain patients from that of healthy control subjects were investigated. These were habituation and genetic factors. Previous research has reported that chronic pain patients habituate to pain stimuli less than pain-free control subjects (Brandt \&Schmidt, 1987; Arntz et al, 1990; Valeriani et al, 2003; Tommaso et al, 2005; Smith et al, 2008). As such, habituation has been posited as an explanation for the development of chronic pain. Chapter six describes a study that investigated whether this difference in habituation is also present in cortical activity. The answer was affirmative. Chronic low back pain patients were found to exhibit less habituation to experimental pain than pain-free control subjects. An interesting finding was that depressive symptoms impact this difference. In fact, the study showed that chronic pain patients with depressive symptoms did not habituate at all. Although the study described in chapter six was crosssectional rather than longitudinal, and thus no conclusive implications about the cause of chronic pain can be made, the findings nonetheless yield interesting and relevant information. First, if chronic pain patients do not (or to a lesser degree) habituate to experimental pain, they probably also do not (or to a lesser degree) habituate to their own clinical pain. It is thus possible that this lack of habituation may in fact be a pain-sustaining factor. Second, given that depressive symptoms were found to influence the habituation of pain stimuli but only in the presence of clinical pain complaints, it is possible that depressive symptoms are in fact the result of the chronic pain complaints. A consequent vicious circle of increasing sensitivity (vigilance) to pain may influence habituation. This idea is supported by literature demonstrating an increased sensitivity to pain in depression (Lautenbacher \& Krieg, 1994; Carter et al, 2002). 
The second factor that may explain differences in cortical processing between chronic pain patients and healthy control subjects is genetics. Studies have reported differences in gene polymorphisms between chronic pain patients and healthy control subjects (for review see, Fillingim et al, 2008). The COMT Val158Met, the BDNF Val66Met and the OPRM1 A118G gene polymorphisms are believed to influence the pain experience (Šerý et al, 2005; Max et al, 2006; Buskila, 2007). The study described in chapter 7 sought to investigate the influence of these gene polymorphisms on the cortical processing of pain. The overall results did not reveal any influence of these gene polymorphisms on the pain-ERP. They also failed to demonstrate differences in allele or genotype distribution between chronic pain patients and healthy control subjects. The results did not replicate previous findings and thus do not support the contention that genetics directly influence pain. However, further (post-hoc) analyses did reveal that the influence of all three gene polymorphisms was moderated by group (chronic pain patients versus healthy control subjects). This suggests that the influence of these gene polymorphisms on the cortical processing of pain may be altered in the presence of chronic pain complaints. The expected painaugmenting effect of the COMT Met allele was found to occur only among chronic pain patients. Among pain-free control subjects, the Met allele was found to have a beneficial effect on the cortical processing of pain. This effect was similar for the BDNF Met allele. The G allele of the OPRM1 polymorphism was hypothesized to have a pain-reducing effect. This was only found when chronic pain complaints were not present. Among chronic pain patients, the OPRM1 G allele had a pain-augmenting, rather than a pain-reducing effect. The results thus demonstrated that the presence of chronic pain complaints moderates the influence of the COMT, BDNF and OPRM1 polymorphisms on the cortical processing of experimental pain stimuli. There are several possible explanations for this effect. First, an explanation concerning the COMT Val ${ }^{158}$ Met polymorphism might be that there are alterations in the endogeneous opioid system that are associated with chronic pain complaints (Bruehl, 1999). Jensen and colleagues (2009) reported that COMT Val ${ }^{158}$ Met differences may be more expressed in individuals where the inhibitory nociceptive system is already challenged and sensitive (Jensen et al, 2009). Second, the effect of chronic pain on cortical processing may be mediated via altering gene expression and chromatin via epigenetic mechanisms. Epigenetics refers to the heritable, but reversible, regulation of various genomic functions, which occurs not by affecting the actual DNA sequence but rather by modifying DNAassociated molecules and chromatin (Jaenisch et al, 2003). Some examples of epigenetic modifications are genetic imprinting, $\mathrm{X}$-inactivation, DNA-methylation and histone modifications such as acetylation and methylation (Dennis, 2003). Recent experimental animal work has indicated that epigenetic mechanisms are involved in the long-lasting effects of chronic exposures to environmental factors 
(McClung\& Nestler, 2008). For example, chronic exposure to social defeat stress in mice was found to significantly down-regulate mRNA levels of histone deacetylase- 5 in the nucleus accumbens (Renthal et al, 2007). Chronic defeat stress in mice furthermore induced lasting down-regulation of BDNF transcripts and increased histone methylation (Tsankova et al, 2006). Interestingly, ongoing treatment with the tricyclic antidepressant imipramine reversed this down-regulation of BDNF transcripts while increasing histone acetylation at the corresponding promoters (Tsankova et al, 2006). Chronic exposures to environmental factors may thus result in dynamic changes to neuronal gene expression which can persist over time but can also be reversed. By exerting effects on gene expression, epigenetic mechanisms have also been shown to influence neuroplasticity (Borrelli et al, 2008). With this in mind, one could indeed hypothesize that chronic pain alters cortical function and neuroplasticity via altering epigenetic mechanisms.

In sum, these findings suggest that differences in habituation between chronic low back pain patients and pain-free control subjects may explain the persistence of pain complaints. Furthermore, the COMT Met allele, the BDNF Met allele and the OPRM1 G allele were all found to increase cortical pain processing in the presence of chronic pain complaints and decrease cortical pain processing in the absence of chronic pain complaints. These gene-environment interactions are perhaps caused by epigenetic mechanisms.

\section{Putting the results together}

An overview of all the study results presented in this dissertation is displayed in figure 1 . This figure shows all the significant relations and interactions. Future research should endeavor to, firstly, replicate the relations and interactions in this figure and, secondly, expand upon the figure with other paininfluencing factors such as personality, pain coping mechanisms and other pain-relevant factors. 


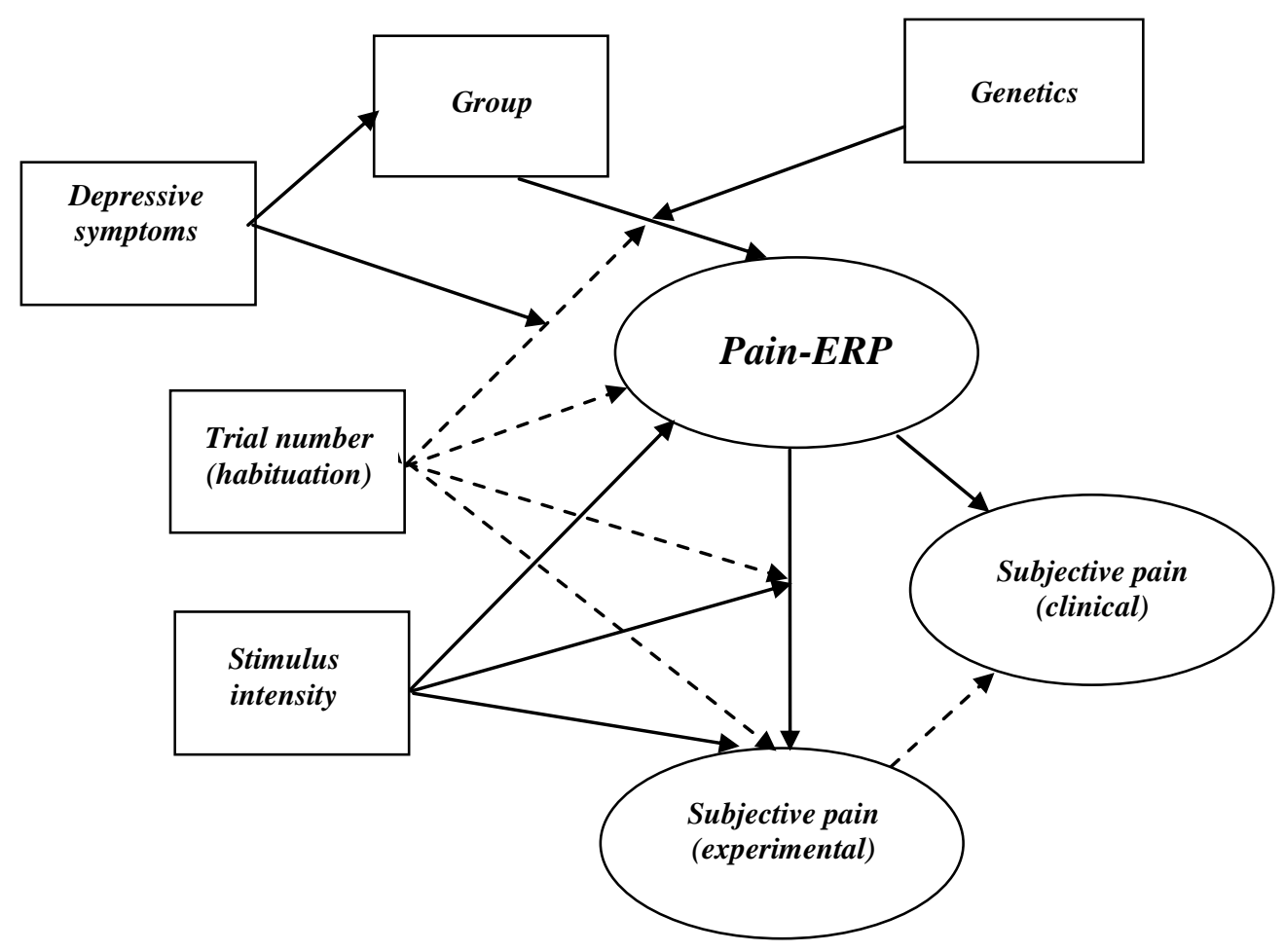

Figure 5 Overview of all the results presented in this dissertation. The solid lines represent a positive association. The dotted lines represent a negative association. Group = chronic pain patients versus pain-free control subjects (coded 1 and 0 , respectively).

\section{Clinical implications of this thesis}

This dissertation presents mostly experimental and fundamental research findings, but which clinical implications can be made? An important aim of this dissertation was to build a bridge between experimental findings and their clinical applications and implications. For this reason, not only pain-free control subjects were tested but also individuals with chronic low back pain. A cross-sectional comparison between these two groups provided insight with respect to how chronic pain complaints influence the processing of new (experimental) pain stimuli. The dysfunction of habituation in chronic pain patients described in chapter six may represent a mechanism by which pain complaints in chronic pain patients persist. In addition, the presence of depressive symptoms was found to augment this dysfunction even further. This provides additional evidence that the treatment of depressive symptoms can positively influence pain complaints. 
Another implication of the present experimental findings to clinical practice is to not only measure experimental pain in laboratories but to also in daily life. The results reported in this dissertation have demonstrated that experimental measures such as ERPs also have clinical value, especially with respect to predicting the amount of daily pain. As stated above, these results are rather preliminary and replication is necessary. However, with further research, it may be possible to use the pain-ERP as a diagnostic tool. This would be particularly useful for patients that cannot (adequately) communicate the amount of pain they experience (e.g. small children, people with dementia or people who are unconscious). A good assessment of the amount of pain experienced by non-communicative patients is essential for adequate pain management (Kwekkeboom \& Herr, 2001). Currently, observational techniques such as the Critical-care Pain Observational Tool (CPOT) (Gelinas et al, 2006) and the Non Verbal Pain Scale (NVPS) (Odhner et al, 2003) are used. In order for these tools to work, comprehensive training is required. Additionally, the observations made are dependent on the person making them thus yielding observational bias. Consequently, the use a psychophysiological measure such as the painERP would be a significant improvement on current practice.

\section{Where to go from here}

The intention of this dissertation was to start unraveling the pain-ERP and provide impetus for additional research. As such, it has not only answered questions but also raised new questions in need of further attention. In this dissertation, the multilevel technique has been established as a promising statistical tool in ERP research. Fine-tuning is, however, necessary. As mentioned in chapter two, variance within the ERP does not only surround the maximum peak amplitudes. Throughout the whole latency range of the ERP curve, there is variance that needs to be explained. Therefore, measures such as the area under the curve or wavelets are an important addition to the maximum peak amplitude of ERP components. Also, wavelet denoising has been found to be useful in separating signal from noise in single trials (Quien Qiuroga, 2000). This dissertation posits that a combination of wavelet denoising and multilevel analyses would be a step forward in the development of a solid technique for the statistical handling of ERPs. Once a solid technique is established, the pain-ERP can continue to be unravelled. The ultimate goal would be to explain as much variance in the pain-ERP as possible. The model displayed in figure 1 is a starting point for this process. More psychological, cultural and environmental factors should to be included and investigated. Once this is accomplished, the pain-ERP will likely no longer be restricted to experimental research but will also be able to enter clinical practice. Lastly, more 
longitudinal research is required in order to obtain additional knowledge about causality. It is important to determine whether differences in habituation between chronic pain patients and pain-free control subjects are trait-related, which is supported by the random slopes of habituation demonstrated in chapter 3, or caused by the presence of chronic pain complaints. Additionally, longitudinal research could likely provide more insight with respect to the relationship between pain and depression. The results presented in this dissertation have not indicated whether depressive symptoms cause or are caused by chronic pain complaints. This should be investigated further.

\section{Concluding remarks}

This dissertation demonstrates that the ERP technique is an appropriate tool that can be employed when conducting etiological pain research. ERP is a suitable and valuable pain measure that contains objective multidimensional information about pain processing. This is necessary for the assessment and investigation of the multidimensional nature of pain, which has been demonstrated in this dissertation by investigating the interaction between somatic (stimulus intensity, habituation, gene polymorphisms) and psychological factors (depressive symptoms). Additionally, multilevel analysis has proven to be an essential tool in unravelling the complex structure of the ERP of long lasting pain. Prospective studies that take the multidimensional nature of pain into account are imperative. 


\section{References}

Arntz A, Lousberg R (1990) The effects of underestimated pain and their relationship to habituation. Behaviour Research and Therapy, 28, 15-28.

Borrelli F, Nestler EJ, Allis CD, Sassone-Corsti P (2008) Decoding the epigenetic language of the neuronal plasticity. Neuron, 60, 961-974.

Brands AM, Schmidt AJ (1987) Learning processes in the persistence behavior of chronic low back pain patients with repeated acute pain stimulation. Pain, 30, 329-337.

Bruehl S, McCubbin JA, Harden RN (1999) Theoretical review: altered pain regulatory systems in chronic pain. Neurosci Biobehav Rev, 23, 877-890.

Buskila D (2007) Genetics of chronic pain states. Best Practice \& Research Clinical Rheumatology, 21, 535-547.

Carlsson (1983) Assessment of Chronic Pain I: aspects of reliability and validity of the Visual Analogue Scale. Pain, 16, 87-101.

Carter LE, McNeil DW, Vowles KE, Sorrell JT, Turk CL, Ries BJ, Hopko DR (2002) Effects of emotion on pain reports, tolerance and physiology. Pain Research \& Management, 7, 21-30.

Chen CAN, Chapman CR, Harkins SW (1979) Brain potentials are functional correlates of induced pain in man. Pain, 6, 365-374.

Cohen TE, Kaplan SW, Kandel ER, Hawkins RD (1997). A simplified preparation for relating cellular events to behavior: Mechanisms contributing to habituation, dishabituation, and sensitization of the Aplysia gill-withdrawal reflex. Journal of Neuroscience, 17, 2886-2899.

Dennis C (2003) Epigenetics and disease: altered states. Nature, 421, 686-688.

De Tommaso M, Libro G, Guido M, Losito L, Lamberti P, et al. (2005) Habituation of single CO2 laser evoked responses during interictal phase of migraine. Journal of Headache Pain, 6, 195-198.

Fabiani M, Gratton G, Federmeier KD (2000) Handbook of Psychophysiology (2nd ed). Edited by J. T. Cacioppo, L. G. Tassinary, and G. G. Bernston. Cambridge University Press: New York. Pp 85-119.

Fillingim RB, Wallace MR, Herbstman DM, Ribeiro-Dasilva M, Staud R (2008) Genetic contributions to pain: a review of findings in humans. Oral Diseases, 14, 673-682.

Garcia-Larrea L, Peyron R, Laurent B, Mauguiere F (1997) Association and dissociation between laser-evoke potentials and pain perception. Neuroreport, 8, 3785-3789.

Gelinas C, Fillion L, Puntillo KA, Viens C, Fortier M (2006) Validation of the critical-care observation tool in adult patients. Americal Journal of Critical Care, 15, 420-427.

Harkins SW, Chapman CR (1978) Cerebral evoked responses to noxious dental stimulation: relationship to subjective pain report. Psychophysiology, 15, 248-252.

Herr KA, Spratt K, Mobily PR, Richardson G (2004) Pain intensity assessment in older adults: use of experimental pain to compare psychometric properties and usability of selected pain scales with younger adults. Clinical Journal of Pain, 20, 207-219. 
Jaenisch R, Bird A (2003) Epigentic regulation of gene expression: how the genome integrates intrinsic and environmental signals. Nature Genetics, 33, 245-254.

Jensen KB, Lonsdorf TB, Schalling M, Kosek E, Ingvar M (2009) Increased Sensitivity to Thermal Pain following a Single Opiate Dose is influenced by the COMT Val158Met Polymorphism. PLoSONE, 4, e6016.

Kwekkeboom KL, Herr K (2001) Assessment of pain in the critically ill. Critical Care Nursing Clinics of North America, 13, 181-194.

Lautenbacher S, Krieg JC (1994) Pain perception in psychiatric disorders: a review of the literature. Journal of Psychiatric Research, 28, 109-122.

Max MB, Wu T, Atlas SJ, Edwards RR, Haythornthwaite JA, Bollettino AF, Hipp HS, McKnight CD, Osman IA, Crawford EN, Pao M, Nejim J, Kingman A, Aisen DC, Scully MA, Keller RB, Goldman D, Belfer I (2006) A clinical genetic method to identify mechanisms by which pain causes depression and anxiety. Molecular Pain, 2, $14-27$.

McClung CA, Nestler EJ (2008) Neuroplasticity mediated by altered gene expression. Neuropsychopharmacology, 33, 3-7.

Mes, C (2007) Improving non-optimal results in chronic pain treatment: A tripartite approach. Dissertation. Gildeprint Drukkerijen: Enschede, the Netherlands.

Miltner W, Larbig W, Braun C (1987) Habituation of subjective rating and event related potentials to painful intracutaneous electrical stimulation of the skin. Journal of Psychophysiology, 1, 221-228.

138 Odher M, Wegman D, Freeland N, Steinmetz A, Ingersoll GL (2003) Assessing pain control in non-verbal critically ill adults. Dimensions of Critical Care Nursing, 22, 260-267.

Quian Quiroga R (2000) Obtaining single stimulus evoked potentials with wavelet denoising. Physica, D145, 278292.

Renthal W, Maze I, Krishnan V, Covington HE $3^{\text {rd }}$, Xiao G, et al. (2007) Histone deacteylase 5 epigenetically controls behavioral adaptations to chronic emotional stimuli. Neuron, 8, 517-529.

Šerý O, Hrazdilová O, Matalová E, Ševčik P (2005) Pain research update from a genetic point of view. Pain Practice, 5, 341-348.

Smith BW, Tooley EM, Montague EQ, Robinson AE, Cosper CJ, Mullins PG (2008) Habituation and sensitization to heat and cold pain in women with fibromyalgia and healthy controls. Pain, 140, 420-428.

Tsankova NM, Berton O, Renthal W, Kumar A, Neve RL, et al. (2006) Sustained hippocampal chromatin regulation in a mouse model of depression and antidepressant action. Nature Neuroscience, 9, 519-525.

Valeriani M, de Tommaso M, Restuccia D, le Pera D, Giudo M, et al. (2005) Reduced habituation to experimental pain in migraine patients: a CO2 laser evoked potential study. Pain, 105, 57-64 




\section{Summary}

Samenvatting 


\section{Summary}

Pain is a subjective experience. This means that not only psychical factors but also psychological, cognitive, social and environmental factors influence the way pain is perceived. This subjective character makes direct measurement of pain impossible. Pain Event-Related Potentials (pain-ERP) represent the cortical response to pain and are thought to be a more objective measure compared to self-report measures such as Visual Analogue Scales. However, the pain-ERP is a rather complex measure which contains considerable variability. This variability most probably is caused by other (pain-related) information which needs to be identified. This thesis discusses the use of Event-Related Potentials in pain research. More specifically, it discusses methodological concerns and ideas about unravelling the pain-ERP.

Chapter 1 discusses the phenomenology and background of pain. It describes the development of different pain theories and how this development has changed over time. The theory that still dominates, the Gate-Control Theory (GCT), is described. The multidimensionality character of pain, which plays a central role in the GCT is discussed. A brief overview of the different brain areas that are involved in the processing of pain, is given. The difficulty in measuring pain as well as how Event-Related Potentials can serve as a more objective measure of pain compared to for instance Visual Analogue Scales, is described. Finally, an outline of the different chapters is given.

Chapter 2 describes a study that led to the formation of the following chapters. This is the preliminary part. The purpose of this study was to examine the influence of neuroticism and its subfacets anxiety and depression on the way pain is processed cortically. Healthy students received painful and non painful electrical stimulation, while EEG was measured. The variance surrounding the ERP curve was explained by neuroticism and its subfacets. Neuroticism significantly moderated the ERP amplitudes, in that higher neuroticism scores were associated with higher ERP amplitudes. Furthermore, differential effects were found for the subfacets: anxiety reduced, whereas depression augmented pain-ERP amplitudes. These findings suggest that a personality trait reflecting bias towards negative emotions may moderate the way pain is processed cortically, with directionally different effects depending on whether the trait is expressed predominantly in the realm of anxiety or depression. 
In Chapter 3 a new way of statistically analyzing Event-Potentials is proposed. Although multilevel analysis is not new, it has not yet been used in ERP research despite the clear theoretical advantages of this technique. Using data from an experimental design, in which pain-free controls with a broad age range received electrical stimulation while they underwent EEG registration, these theoretical advantages were tested in practice. The ability in multilevel analysis to include single trial data and to model beyond polynomial contrasts, led to the finding that the amplitude of specific pain components of the ERP tend to follow an inverse (1/ $x$ ) decreasing pattern (habituation) across trials. Furthermore, the ability to test for random slopes resulted in the significant finding that individuals differ in their response across stimulus intensities and differ in their habituation pattern. Finally, because of the ability to include single trial data, it was possible to test within-subject covariates and this led to the finding that the intensity of the previous stimulus is in interaction with the intensity of the present stimulus. In other words, how persons cortically react to certain stimulus intensity, depends on the intensity of the stimulus given previous to the present one. The general conclusion was that the multilevel technique is a promising technique for explaining the variance surrounding the ERP curve.

In Chapter 4 the relation between specific pain peak components of the ERP and the subjective experience of pain measured with Numeric Rating Scales is investigated. Using the multilevel technique, described in chapter 3 it was attempted to get a more detailed view on this relation compared to the bivariate correlations found in scientific literature. The results from previous studies could be replicated, though, in a weaker fashion. The multilevel technique did reveal that the relation between ERP peak components and the subjective experience of pain was moderated by stimulus intensity and trial (habituation). These results suggest that a direct translation from the pain-ERP to subjective experience is delicate and that factors such as stimulus intensity and habituation must be taken into account.

Chapter 5 further explores the relation between the pain-ERP and subjective pain. However, in this chapter the subjective ratings of clinical pain are investigated. The aim of this study is to explore whether the pain-ERP measured in the laboratory has predictive value for clinical pain experience in daily life. Chronic low back pain patients filled in a two-week registration of their pain complaints. At baseline pain-ERPs were measured. The analyses reveal that not only the subjective ratings of the experimental pain stimuli (NRS), but, most interestingly, also the N2-component at Cz and C4 of the pain-ERP, significantly predict the subjective experience of clinical pain in daily life. Furthermore, when the N2 components and the NRS were corrected for each other, the ERP measures remained significant 
predictors, whereas the subjective NRS did not. This study demonstrates that the pain ERP measured in an experimental laboratory setting has predictive value for clinical pain in daily life.

In Chapter 6 cortical differences between chronic low back pain patients and pain free controls are investigated. More specifically, it is demonstrated that chronic low back pain patients show less habituation over trials compared to pain free controls. This suggests that chronic pain patients may perceive a painful stimulus longer as painful than pain free controls. Furthermore, depressive symptoms were found to moderate this difference in habituation. In pain free controls, depressive symptoms have no effect on habituation, while in chronic low back pain patients they lead to a complete absence of habituation. One could speculate that this dysfunction in habituation might cause the development or perseverance of chronic pain complaints. Longitudinal data is needed to validate this speculation.

Chapter 7 describes a study that investigated the influence of specific gene polymorphisms on the cortical processing of pain. The COMT Val158Met, BDNF Val66Met and OPRM1 A118G polymorphisms are hypothesized to be pain gene candidates. The results of this study could not replicate previous findings in which a difference in allele or genotype distribution was found between chronic pain patients and pain free controls. Neither, differences in cortical pain processing between the alleles were found. However, it was found that the influence of these polymorphisms on the cortical pain processing was moderated by the presence of chronic pain complaints. Only in the presence of chronic pain complaints did the expected pain augmenting effect of the Met allele of the COMT and BDNF polymorphism occur. Furthermore, in the presence of chronic pain complaints the expected pain reducing effect of the $G$ allele of the OPRM1 polymorphisms turned into a pain augmenting effect. So the influence of genes on the cortical processing of pain is different for chronic pain patients than for pain free controls.

Chapter 8 discusses and summarizes the results described in the previous chapters. A model is presented which combines all the different results. An attempt is made to bridge the gap between experimental findings and clinical applications in the use of Event-Related Potentials. Finally, directions for future research are provided. 


\section{Samenvatting}

Pijn is een subjectieve ervaring. Dit betekent dat niet alleen fysieke factoren maar ook psychologische, cognitieve, sociale en omgevings factoren invloed hebben op de ervaring van pijn. Vanwege het subjectieve karakter van pijn is het onmogelijk om pijn direct te meten. Pijn 'Event-Related Potentials' (pijn-ERP) zijn weergaven van de corticale verwerking van pijn en worden gezien als een objectievere maat van pijn in vergelijking tot zelfrapportage, met behulp van bijvoorbeeld visuele analoge schalen. Echter, het pijn-ERP is een complexe maat die aanzienlijk veel variabiliteit bevat. Deze variabiliteit wordt hoogstwaarschijnlijk veroorzaakt door andere (pijn-gerelateerde) informatie die geïdentificeerd dient te worden. Dit proefschrift bespreekt het gebruik van Event-Related Potentials in pijnonderzoek en meer specifiek, methodologische kritiekpunten en ideeën over het ontrafelen van het pijn-ERP.

Hoofdstuk 1 betreft de algemene introductie van dit proefschrift. Het bespreekt de fenomenologie en achtergrond van pijn. Het beschrijft de ontwikkeling van verschillende pijn theorieën en hoe deze veranderden door de eeuwen heen. De theorie die tegenwoordig het meest wordt aangehouden, de Gate Control Theory (GCT), wordt uitvoerig in dit hoofdstuk besproken. Verder wordt het multidimensionele karakter van pijn, dat een centrale rol speelt in de GCT, behandeld. Een kort overzicht wordt gegeven van de verschillende hersengebieden die actief zijn bij de verwerking van pijn. Daarnaast wordt de moeilijkheid van het meten van pijn besproken en hoe Event-Related Potentials als objectievere maat van pijn ten opzichte van bijvoorbeeld Visual Analogue Scales kunnen dienen. Ten slotte geeft de algemene introductie een overzicht van de verschillende hoofdstukken van dit proefschrift.

Hoofdstuk 2 beschrijft een studie die leidde tot de totstandkoming van de navolgende hoofdstukken. Dit is de proloog. Het doel van deze studie was om de invloed van neuroticisme en twee onderliggende subfacetten (angst en depressie) op de corticale verwerking van pijn te onderzoeken. Gezonde studenten ontvingen pijnlijke en niet-pijnlijke stimuli, terwijl het EEG werd gemeten. De variantie rondom de ERP curves kon worden verklaard door neuroticisme en de subfacetten. Een significant hoofdeffect van neuroticisme liet zien dat personen met hogere neuroticisme scores, hogere ERP amplitudes hadden. Daarnaast werden er tegenovergestelde effecten van de subfacceten angst en depressie op het pijn-ERP gevonden: angst was gerelateerd aan lage amplitudes, terwijl depressie gerelateerd was aan hogere amplitudes. Deze resultaten suggereren dat een persoonlijkheidstrek, die een neiging tot het ervaren van negatieve emoties representeert, de corticale verwerking van pijn 
beïnvloedt, waarbij de richting van het effect afhankelijk is van of deze trek overwegend geuit wordt in het subfacet angst of depressie.

In hoofdstuk 3 wordt een nieuwe manier gepresenteerd om Event-Related Potentials statistisch te analyseren. Deze manier is multilevel regressie ook wel mixed regression genoemd. Hoewel multilevel regressie geen nieuwe techniek is, is het tot nu toe nog niet gebruikt in de analyse van ERPs. Dit is opmerkelijk aangezien multilevel regressie op theoretische basis duidelijke voordelen biedt. In dit hoofdstuk worden deze theoretisch voordelen in de praktijk getest met behulp van data uit een experimentele studie waarbij gezonde proefpersonen, met uiteenlopende leeftijden, elektrische stimulatie kregen toegediend terwijl EEG werd gemeten. De mogelijkheid om in multilevel analyses losse trials te includeren en meer dan alleen polynomiale contrasten te modelleren, heeft tot de bevinding geleid dat de amplitude van pijn specifieke componenten van het ERP een niet lineair patroon van habituatie volgen over trials. Daarnaast, de mogelijkheid om 'random slopes' te modeleren heeft geresulteerd in de bevinding dat individuen verschillen van elkaar in de mate van habituatie. Tenslotte, het feit dat multilevel regressie data van losse trials kan analyseren heeft het mogelijk gemaakt om binnenpersonen covariantie te analyseren en dit leidde to de bevinding van een interactie tussen stimulus intensiteit van de huidige prikkel en stimulus intensiteit van de voorgaande prikkel. Dit betekent dat hoe personen corticaal reageren op een bepaalde stimulus intensiteit, afhankelijk is van de intensiteit van de voorgaande prikkel. De algemene conclusie van dit hoofdstuk is dat de multilevel regressie techniek een veelbelovende techniek is voor het verklaren van de variantie die om de ERP curve bestaat.

In hoofdstuk 4 wordt de relatie onderzocht tussen specifieke pijn componenten van het ERP en de subjectieve ervaring van pijn, gemeten met de Numeric Rating Scale (numerieke scores). Met behulp van de multilevel techniek, beschreven in hoofdstuk 3, is getracht om meer getailleerde informatie te verkrijgen over deze relatie dan de bivariate correlaties die gevonden zijn in de literatuur. De resultaten van de literatuur konden gerepliceerd worden in de huidige studie, hoewel de effecten minder sterk leken. De multilevel regressie heeft verder laten zien dat de relatie tussen het ERP en de subjectieve ervaring van pijn gemodereerd wordt door stimulus intensiteit en habituatie (de hoeveelste prikkel). Deze resultaten geven aan dat men voorzichtig moet zijn met een directe vertaling van het pijn ERP naar subjectieve ervaring van pijn en dat er rekening dient te worden gehouden met factoren zoals stimulus intensiteit en habituatie. 
Hoofdstuk 5 gaat verder met het onderzoek naar de relatie tussen het pijn-ERP en de ervaring van pijn. In dit hoofdstuk wordt echter de subjectieve ervaring van klinische pijn onderzocht. Het doel van de studie beschreven in dit hoofdstuk, is om te onderzoeken of het pijn-ERP, dat gemeten is in het lab, een voorspellende waarde heeft voor de ervaring van klinische pijn in het dagelijks leven. Chronische lage rugpijn patiënten vulden gedurende twee weken elke dag een vragenlijst in over hun pijn klachten. Bij het begin werd tevens een pijn-ERP gemeten. The analyses lieten zien dat naast een sterk voorspellende waarde van de subjectieve ervaring van experimentele pijn (NRS), ook het pijn-ERP (N2-component op $\mathrm{Cz}$ en (4) een sterk voorspellende waarde heeft voor de ervaring van pijn in het dagelijks leven. Daarnaast, wanneer de NRS en het pijn-ERP voor elkaar werden gecorrigeerd, bleek dat het pijn-ERP een significante voorspeller bleef, terwijl dit niet het geval was voor de NRS. Deze studie demonstreert dat het pijn-ERP, gemeten in een laboratorium, predictieve waarde heeft voor klinische pijn in het dagelijks leven.

In hoofdstuk 6 worden corticale verschillen tussen chronische lage rugpijn patiënten en pijnvrije controles onderzocht. Specifiek wordt er gedemonstreerd dat chronische lage rugpijn patiënten minder habituatie vertonen over meerdere trials in vergelijking tot controles. Dit lijkt aan te geven dat chronische pijn patiënten een pijnlijke stimulus als langer pijnlijk ervaren dan pijnvrije controles. Daarnaast werd gevonden dat depressieve klachten hier een interacterend effect op hebben. In pijnvrije controles hebben depressieve klachten geen effect op de gewenning aan prikkels maar bij chronische pijn patiënten zorgen depressieve klachten voor een nog mindere habituatie van de prikkels. Men zou kunnen denken dat deze disfunctie in habituatie de oorzaak is van het ontstaan en in stand houden van chronische pijnklachten. Echter, longitudinaal onderzoek is nodig om dit te bevestigen.

Hoofdstuk 7 beschrijft een studie naar de invloed van specifieke gen polymorfismen op de corticale verwerking van pijn. De COMT Val158Met, BDNF Val66Met en OPRM1 A118G polymorfismen zijn gehypothetiseerde pijn gen kandidaten. De resultaten van deze studie konden niet eerder bevindingen repliceren waarin een verschil in allel of genotype distributie werd gevonden tussen chronische pijn patiënten en pijnvrije controles. Daarnaast werden er ook geen verschillen in corticale pijnverwerking gevonden tussen risico allel dragers en de 'niet-dragers'. Wat echter wel werd gevonden was dat de invloed van deze genpolymorfismen op de corticale pijnverwerking werd gemodereerd door het al dan niet hebben van chronische pijnklachten. Alleen in de aanwezigheid van chronische pijnklachten werd het verwachte pijnversterkende effect van het Met allel van zowel het COMT als BDNF polymorfisme gevonden. Daarnaast veranderde, in de aanwezigheid van chronische pijnklachten, het verwachte 
pijndempende effect van het $\mathrm{G}$ allel van het OPRM1 genpolymmorfisme in een pijnversterkend effect. Deze resultaten geven aan dat de invloed van genen op de verwerking van pijn in de hersenen verschillend is voor chronische pijnpatiënten als voor pijnvrije mensen.

Hoofdstuk 8 bespreekt de resultaten van de voorgaande hoofdstukken en geeft een samenvatting. Een model wordt gepresenteerd waarin alle resultaten worden gecombineerd. Een poging wordt gedaan om een brug te slaan tussen experimentele bevindingen en klinische toepassingen van het ERP. Tenslotte worden aanwijzingen voor gegeven toekomstig onderzoek. 


\section{Curriculum Vitae}

Hélène Guillaume Marie Vossen werd geboren op 26 januari 1981 in Geleen. In 1999 behaalde ze het VWO diploma aan het Serviam in Sittard. Vervolgens ging zijn naar Maastricht om daar aan de Universiteit psychologie te studeren. Haar wetenschappelijke afstudeerstage deed zij aan de vakgroep Psychiatrie en Neuropsychologie van de Universiteit Maastricht. Gelijktijdig werkte ze daar als studentassistent bij de MIND-IT studie. In 2004 rondde zijn haar studie Psychologie, met als afstudeerrichting Neuropsychologie af. Direct hierna begon ze aan haar promotieonderzoek, met als onderwerp de corticale verwerking van pijn. Dit betrof een samenwerking tussen de vakgroep Psychiatrie en Neuropsychologie van de Universiteit van Maastricht en het RRD (Roessingh Research and Development) in Enschede. Daarnaast werkte zijn enige tijd als psycholoog bij de MedPsychUnit van het Academisch Ziekenhuis van Maastricht. Sinds 1 september 2009 werkt Helen Vossen aan de 'Universitat de Barcelona', alwaar zij statistische begeleiding geeft bij onderzoek naar gen-omgeving interacties in psychose en schizofrenie. 



\section{Dankwoord}

De totstandkoming van dit proefschrift is mede mogelijk gemaakt door de hulp en steun van vele mensen. Ik wil graag een blijk van dank geven aan deze mensen.

Jim van Os, mijn eerste promotor, bedankt voor je vertrouwen in mij en je enthousiasme. Ik waardeer je snelle reacties op mijn artikelen en je enorme kennis. Verder wil ik je heel erg bedanken voor je hulp bij de ontwikkeling van mijn carrière. Hermie Hermens, mijn tweede promotor, ik denk met veel plezier terug aan onze lunchafspraken in het Roessingh. Bedankt voor de fijne gesprekken die niet alleen maar over de praktische dingen van het onderzoek gingen.

Richel, mijn copromotor en kamergenoot, ik durf met zekerheid te zeggen dat zonder jou dit proefschrift er echt niet zou liggen. Jij bent veel meer dan alleen mijn copromotor geweest. Ik wil je bedanken voor alle goede gesprekken én voor alle 'ruzies'. Je hebt mij niet alleen de kneepjes van het vak geleerd maar ook wat kneepjes van het leven. Je bent een onderzoeker pur sang en een voorbeeld voor mij.

De leden van de beoordelingscommissie wil hartelijk danken voor het beoordelen van mijn proefschrift.

De co-auteurs van mijn artikelen, Gerard van Breukelen, Gunter Kenis, Carsten Leue en Bart Rutten, wil ik bedanken voor de prettige samenwerking en waardevolle input. Gerard, ik hoop dat ik de kennis die ik bij jou heb opgedaan goed over kan brengen in mijn nieuwe baan.

Claudy, Suzanne, Yvonne, Heleen, Kim en Daisy, ik wil jullie heel erg bedanken voor al jullie hulp met de dataverzameling en data-invoer van het onderzoek. Jullie meiden hebben heel hard gewerkt en geleerd dat in het onderzoek niet altijd alles volgens plan verloopt. ik heb genoten van jullie gezelschap.

Sarah, jou wil ik bedanken voor je hulp als student assistent en als corrigeerder van het Engels van van dit proefschrift. Ik heb nog nooit iemand gezien die zo doelgericht en efficiënt te werk gaat.

Bedankt al mijn collega's aan de vakgroep Psychiatrie en Neuropsychologie. Ron en Nico, bedankt voor jullie enorme hulp met mijn laptop en ander technische aspecten. Jacqueline, Carsten en Ruud, 
bedankt voor de mogelijkheden die jullie me hebben gegeven op de MedPscyhUnit. Ik heb hier heel veel ervaring opgedaan. Franz, je hebt me veel geleerd over diagnostiek. Ik waardeer enorm de tijd en moeite die je hebt vrijgemaakt voor mij. Dames van het secretariaat, Margot, Vivianne, Wendy, Kirsten, Els, Maddy, Annemie en Aggie, bedankt voor de gezellige tussendoortjes en voor jullie hulp. Ik vrees dat ik het faxen helaas nog steeds niet helemaal onder de knie heb. Ankie, Laurent en Marco, bedankt voor al jullie hulp aangaande alle financiële zaken.

Al mijn lieve vrienden wil ik bedanken voor hun aanwezigheid in mijn leven. Als verslaafde aan het Bourgondische level hebben jullie mij bijgestaan in mijn behoefte aan lekker eten, lekker drinken en gezelligheid. Jullie hebben geen idee wat jullie voor mij betekenen.

Carine en Christine, mijn paranimfen en liefste zussen van de hele wereld, het geeft me een geweldig gevoel van rust dat jullie straks achter mij zullen staan tijdens de verdediging. Wat betreft persoonlijkheid alsmede professie vormen we een perfecte drie-eenheid. Ik wil jullie bedanken voor jullie enthousiasme, steun en gewoon voor het feit dat jullie mijn zussen zijn.

Dankjewel lieve papa en mama. Jullie zijn mijn steun en toeverlaat. Bedankt voor jullie onvoorwaardelijke steun, vertrouwen en liefde. Dankjewel lieve John en Yvonne. Ook jullie geven mij een gevoel van thuis.

Tenslotte, dankjewel lieve Erik voor al je liefde, steun en vooral humor. 


\section{List of Publications}

\section{Published papers}

Vossen HGM, Van Os J, Hermens H, Lousberg R (2010) The predictive value of pain Event-related potentials for clinical experience of pain. In Press.

Smeets IAP, Tan EYL, Vossen HGM, Leroy PLJM, Lousberg R, Van Os J, Schieveld JNM (2009) Prolonged stay at the paediatric intensive care unit associated with paediatric delirium. European Child \& Adolescent Psychiatry, Epub ahead of print.

Vossen HGM, Monsieur D, Van Os J, Leue C (2009) Een systematisch overzichtsartikel over de plaats van Serotonine en Noradrenaline Reuptake Inhibitoren in de behandeling van chronische niet maligne pijnsyndromen. Tijdschrift voor Psychiatrie, 51, 831-840.

Honig A, Kuyper AMG, Schene AH, van Melle JP, de Jonge P, Tulner DM, Schins A, Crijns HJGM, Kuijpers PMJC, Vossen HGM, Lousberg R, Ormel J (2007) Treatment of Post Myocardial Infarction Depressive Disorder: a Randomized Placebo Controlled Trial with Mirtazapine. Psychosomatic medicine, 69, 606-613 .

Vossen HGM, Van Os J, Hermens H, Lousberg R (2006) Evidence That Trait-Anxiety and TraitDepression Differentially Moderate Cortical Processing of Pain. Clinical Journal of Pain, 22, 725-729.

\section{Submitted papers}

Vossen HGM, Van Breukelen GJP, Hermens H, Van Os J, Lousberg R (2010) More Potential in Statistical Analyses of Event-Related Potentials: A Multilevel Approach. Submitted.

Vossen HGM, Van Breukelen GJP, Van Os J, Hermens H, Lousberg R (2010) A challenge to the notion of a clear-cut association between Event-Related Potentials and subjective pain ratings: An experimental study using multilevel analyses. Submitted. 
Vossen HGM, Van Breukelen, GJP, Hermens H, Van Os J, Lousberg R (2010) Cortical differences in habituation to experimental pain between chronic low back pain sufferers and pain-free controls: the role of depressive symptoms. Submitted.

Vossen HGM, Kenis GR, Rutten BP, Van Os J, Hermens H, Lousberg R (2010) The Influence of COMT 158ValMet, BDNF Val66Met and OPRM1 A118G polymorphisms on the cortical processing of experimental pain. Submitted. 